\title{
Beyond Consensus: Embracing Heterogeneity in Curated Neuroimaging Meta-Analysis
}

Gia H. Ngo ${ }^{1,2}$, Simon B. Eickhoff ${ }^{3,4}$, Minh Nguyen ${ }^{1}$, Gunes Sevinc ${ }^{5}$ Peter T. Fox ${ }^{6,7}$, R. Nathan Spreng ${ }^{8,9}$, B.T. Thomas Yeo ${ }^{1,10,11,12}$

${ }^{1}$ Department of Electrical and Computer Engineering, ASTAR-NUS Clinical Imaging Research Centre, N.1 Institute for Health and Memory Networks Program, National University of Singapore, Singapore ${ }^{2}$ School of Electrical and Computer Engineering, Cornell University, Ithaca, New York ${ }^{3}$ Institute for Systems Neuroscience, Medical Faculty, Heinrich Heine University Düsseldorf, Düsseldorf, Germany ${ }^{4}$ Institute of Neuroscience and Medicine, Brain \& Behaviour (INM-7), Research Centre Jülich, Jülich, Germany ${ }^{5}$ Department of Psychiatry, Massachusetts General Hospital, Harvard Medical School, Boston, MA, USA ${ }^{6}$ Research Imaging Institute, University of Texas Health Science Center at San Antonio, San Antonio, TX, USA ${ }^{7}$ South Texas Veterans Health Care System, San Antonio, TX, USA ${ }^{8}$ Laboratory of Brain and Cognition, Montreal Neurological Institute, McGill University, Montreal, QC, Canada ${ }^{9}$ Departments of Psychiatry and Psychology, McGill University, Montreal, QC, Canada ${ }^{10}$ Martinos Center for Biomedical Imaging, Massachusetts General Hospital, Charlestown, MA, USA ${ }^{11}$ Centre for Cognitive Neuroscience, Duke-NUS Medical School, Singapore ${ }^{12}$ NUS Graduate School for Integrated Sciences and Engineering, National University of Singapore, Singapore

\section{Address correspondence to:}

B.T. Thomas Yeo

ECE, ASTAR-NUS CIRC, N.1 Institute \& MNP

National University of Singapore

Email: thomas.yeo@nus.edu.sg 


\begin{abstract}
Coordinate-based meta-analysis can provide important insights into mind-brain relationships. A popular approach for curated small-scale meta-analysis is activation likelihood estimation (ALE), which identifies brain regions consistently activated across a selected set of experiments, such as within a functional domain or mental disorder. ALE can also be utilized in meta-analytic co-activation modeling (MACM) to identify brain regions consistently coactivated with a seed region. Therefore, ALE aims to find consensus across experiments, treating heterogeneity across experiments as noise. However, heterogeneity within an ALE analysis of a functional domain might indicate the presence of functional sub-domains. Similarly, heterogeneity within a MACM analysis might indicate the involvement of a seed region in multiple co-activation patterns that are dependent on task contexts. Here, we demonstrate the use of the author-topic model to automatically determine if heterogeneities within ALE-type meta-analyses can be robustly explained by a small number of latent patterns. In the first application, the author-topic modeling of experiments involving self-generated thought $(\mathrm{N}=179)$ revealed cognitive components fractionating the default network. In the second application, the author-topic model revealed that the left inferior frontal junction (IFJ) participated in multiple task-dependent co-activation patterns $(\mathrm{N}=323)$. Furthermore, the author-topic model estimates compared favorably with spatial independent component analysis in both simulation and real data. Overall, the results suggest that the author-topic model is a flexible tool for exploring heterogeneity in ALE-type meta-analyses that might arise from functional sub-domains, mental disorder subtypes or task-dependent co-activation patterns. Code for this study is publicly available (https://github.com/ThomasYeoLab/CBIG/tree/master/stable _projects/metaanalysis/Ngo2019_AuthorTopic).
\end{abstract}

\title{
Keywords:
}

theory of mind, autobiographical memory, executive function, inhibition, attentional control, mental disorder subtypes 


\section{Introduction}

Brain imaging experiments are often underpowered (Carp, 2012; Poline et al., 2012; Button et al., 2013). Coordinate-based meta-analysis provides an important framework for analyzing underpowered studies across different experimental conditions and analysis piplines to reveal reliable trends (Wager et al. 2003; Fox et al. 2014; Poldrack and Yarkoni, 2016). Large-scale coordinate-based meta-analyses synthesize thousands of experiments across diverse experimental designs to discover broad and general principles of brain organization and disorder (Laird et al., 2011; Poldrack et al., 2011; Crossley et al., 2014). By contrast, the vast majority of meta-analyses involve smaller number of experiments that are expertly chosen (curated) to generate consensus on specific functional domains (e.g., Binder et al. 2009), brain regions (e.g., Shackman et al., 2011) or disorders (e.g., Cortese et al., 2012).

A popular approach for smaller-scale meta-analyses is activation likelihood estimation or ALE (Laird et al., 2005; Eickhoff et al. 2009, 2012; Turkeltaub et al. 2012). ALE identifies brain regions consistently activated across neuroimaging experiments within a functional domain (Costafreda et al., 2008; Spaniol et al., 2009; Beissner et al., 2013) or within a disorder (e.g., Fitzgerald et al., 2008; Minzenberg et al., 2009; Di Martino et al., 2009). Thus, ALE treats heterogeneities across studies as noise. Consequently, ALE analysis might miss out on genuine biological heterogeneity indicative of functional sub-domains or disorder subtypes.

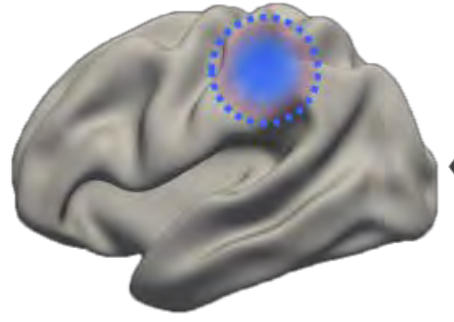

Consensus across experiments (e.g., activation likelihood estimation (ALE))

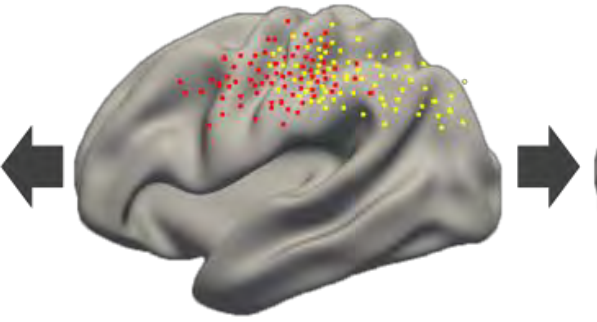

$1 / 2$ experiments reported red activation foci, $1 / 2$ experiments reported yellow foci

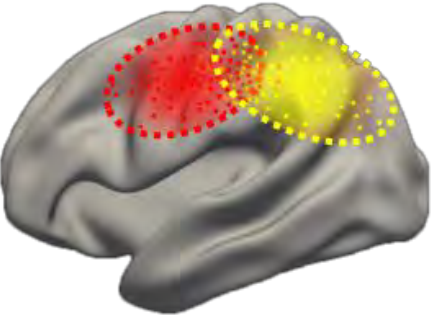

Overlapping activation patterns using author-topic model reveals functional sub-domains

Figure 1. Example of heterogeneity in neuroimaging meta-analysis. The middle panel shows activation peaks reported from neuroimaging experiments within a functional domain. Half the experiments are red dots; half the experiments are yellow dots. The left panel illustrates a possible outcome of activation likelihood estimation (ALE), which converges on regions consistently activated across experiments (blue dotted circle). The right panel illustrates a possible estimate by the author-topic model (Yeo et al., 2015), which recovers overlapping patterns (red and yellow ovals) corresponding to two functional sub-domains. We note that the spatial spread of the activation foci was exaggerated to accentuate the overlaps and differences between the activation patterns of the two functional sub-domains. 
For example, Figure 1 (middle panel) illustrates activation foci from experiments associated with a hypothetical functional domain. These foci are generated by two latent subdomains activating distinct, but overlapping, brain regions. Without prior knowledge of the two sub-domains from theory or previous empirical work, ALE will converge on regions commonly activated across both sub-domains (Figure 1 left panel). To get around this issue, meta-analytic studies can sub-divide experiments into hypothetical functional sub-domains before applying ALE. For example, a recent meta-analysis divided working memory experiments into verbal versus non-verbal tasks, as well as tasks involving object identity versus object locations (Rottschy et al., 2012). However, manually subdividing experiments requires prior knowledge of the sub-domains and may reinforce biases towards existing concepts. By contrast, in this study, we explored whether a previously published data-driven approach (author-topic model; Yeo et al., 2015) can help uncover heterogeneities ${ }^{1}$ within ALEtype meta-analyses in a bottom-up, data-driven fashion (Figure 1 right panel).

\subsection{Discovering sub-domains of self-generated thought}

A good example in which ALE might miss out on functional sub-domains is the default network and self-generated thought (Smallwood, 2013; Andrews-Hanna et al., 2014). Selfgenerated thought involves associative and constructive processes that take place within an individual, and depends upon an internal representation to reconstruct or imagine a situation, understand a stimulus, or generate an answer to a question. The term "self-generated thought" serves to contrast with thoughts where the primary referent is based on immediate perceptual input. By virtue of being largely stimulus independent or task unrelated, self-generated thought has been linked with the functions of the default network (Buckner et al., 2008; AndrewsHanna et al., 2014). Previous ALE meta-analyses have implicated the default network in many tasks involving self-generated thought, including theory of mind, narrative fiction, autobiographical memory and moral cognition (Spreng et al. 2009; Binder et al. 2009; Mar, 2011; Sevinc and Spreng, 2014).

\footnotetext{
${ }^{1}$ We note that when estimating functional sub-domains, we are not interested in capturing idiosyncrasies of individual experiments or even individual tasks. Instead, we are hoping to estimate a small number of overlapping, but distinct activation patterns (cognitive processes) that are recruited to different extents across tasks.
} 
However, many studies have suggested that the default network might be fractionated into sub-systems. For example, Andrews-Hanna and colleagues have proposed a dorsomedial prefrontal subsystem preferentially specialized for social cognition and narrative processing (Andrews-Hanna et al., 2014; Spreng \& Andrews-Hanna, 2015) and a medial temporal lobe sub-system preferentially specialized for mnemonic constructive processes (Andrews-Hanna et al., 2014; Christoff et al., 2016). Both sub-systems might spatially overlap or inter-digitate across multiple brain regions (Andrews-Hanna et al., 2014; Braga and Buckner, 2017), which would be challenging to ALE without assuming prior knowledge of the sub-systems (Figure 1). Furthermore, specific default network fractionation details differed across studies (Laird et al. 2009; Andrews-Hanna et al., 2010; Mayer et al. 2010; Humphreys et al., 2015; Kernbach et al., 2018), so application of the author-topic model might potentially clarify sub-systems subserving self-generated thought.

\subsection{Discovering multiple co-activation patterns of the left inferior frontal junction (IFJ)}

Another common application of ALE is meta-analytic connectivity modeling (MACM), which identifies brain regions that consistently co-activate with a particular seed region (Toro et al., 2008; Koski and Paus, 2010; Robinson et al., 2010; Eickhoff et al., 2010). The assumption is that the seed region exhibits a single co-activation pattern regardless of the actual task activating the seed region (Robinson et al. 2010). However, studies have shown the existence of multiple hub regions in the brain (e.g., dorsal anterior insula, dorsal anterior cingulate cortex) that are activated across many different tasks and might adapt their connectivity pattern depending on task context (Cole et al., 2013; Uddin 2015; Bertolero et al., 2017). Thus, a seed region might be involved in multiple task-dependent co-activation patterns (McIntosh, 2000).

A good example in which MACM might miss out on multiple co-activation patterns is the left inferior frontal junction (IFJ; Muhle-Karbe et al., 2015). The IFJ has been implicated in many cognitive processes (Brass et al. 2005; Chikazoe et al. 2009; Asplund et al. 2010) and is a key node of the multiple-demand system (Duncan et al., 2010; Fedorenko et al., 2010). IFJ might also coordinate information among modules by adapting its connectivity patterns across different resting and task states (Cole et al., 2013; Bertolero et al., 2018). Therefore, one might expect the IFJ region to exhibit multiple co-activation patterns that are dependent on task contexts. Since ALE cannot capture heterogeneity across experiments, MACM might be insensitive to such task-dependent co-activation patterns. On the other hand, application of the author-topic model to the IFJ region might yield multiple meaningful co-activation patterns. 


\subsection{Author-topic model}

In this work, we propose the use of the author-topic model to automatically make sense of heterogeneity within ALE-type meta-analyses. We have previously utilized the author-topic model (Figure 2; Yeo et al. 2015; Bertolero et al., 2015) to encode the intuitive notion that a behavioral task recruits multiple cognitive components, which are in turn supported by overlapping brain regions (Poldrack 2006; Leech et al. 2012; Barrett and Satpute, 2013). While our previous work focused on large-scale meta-analysis across many functional domains (Yeo et al. 2015; Bertolero et al., 2015), the current study focuses on heterogeneity within a functional domain (self-generated thought) or co-activation heterogeneity of a seed region (left IFJ). These applications of the author-topic model are made possible by the development of a novel inference algorithm for the author-topic model (Ngo et al., 2016) that is sufficiently robust for smaller-scale meta-analyses.

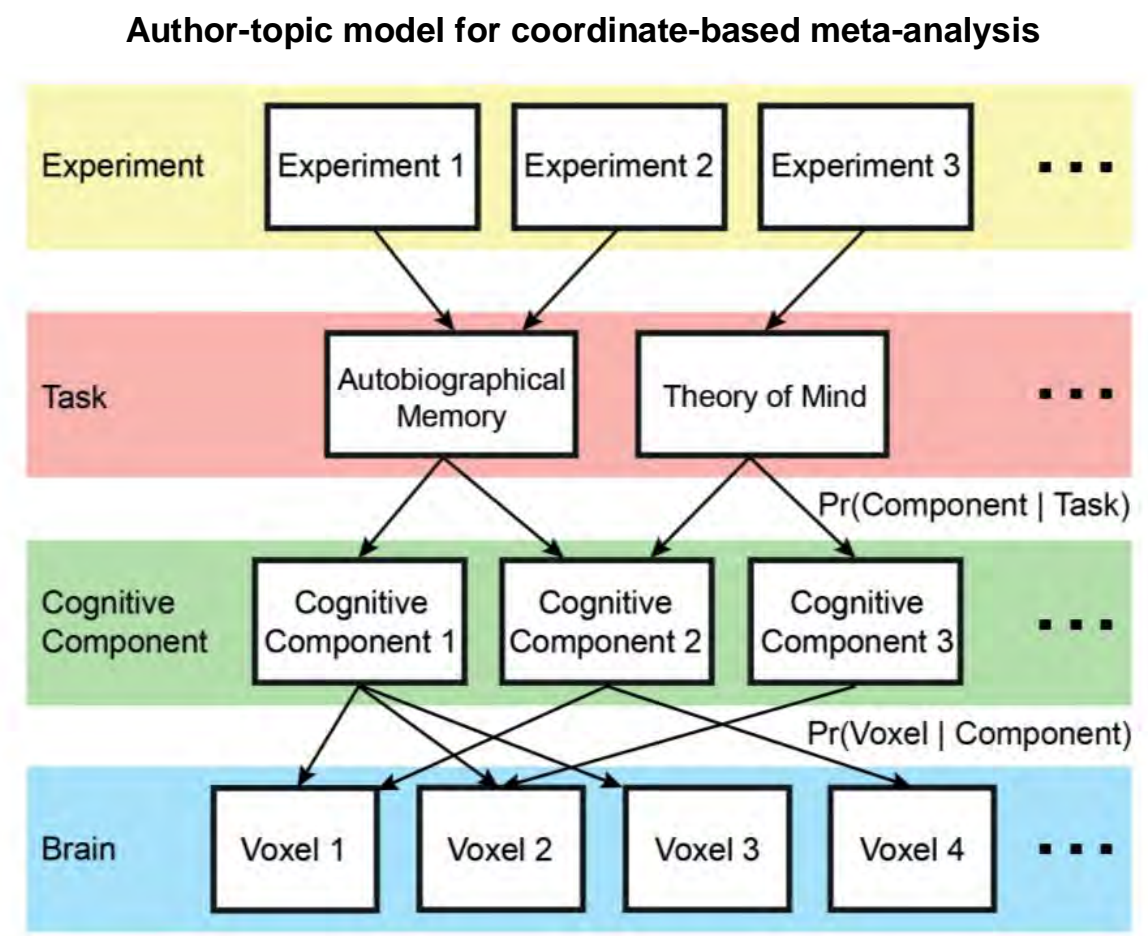

Figure 2. Author-topic model for coordinate-based meta-analysis (Yeo et al. 2015). The underlying premise of the model is that behavioral tasks recruit multiple cognitive components, which are in turn supported by overlapping brain regions. The model parameters are the probability that a task would recruit a cognitive component $(\operatorname{Pr}($ component $\mid$ task $))$ and the probability that a component would activate a brain voxel ( $\operatorname{Pr}(\operatorname{voxel} \mid$ component)). The authortopic model can be directly applied to estimate cognitive components (sub-systems) of selfgenerated thought. 
Our choice of self-generated thought is motivated by previous work suggesting the possibility of fractionating self-generated thought into functional sub-domains (Section 1.1). Similarly, our choice of left IFJ is motivated by previous work suggesting that IFJ might adaptively modify its connectivity patterns across task contexts (Section 1.2). There are of course other functional domains (e.g., executive function) that might be fractionated and other hub regions (e.g., dorsal anterior insula) that might exhibit task-dependent co-activation patterns. Therefore, we have made our code publicly available for researchers to explore the heterogeneity of their preferred functional domain, hub region or mental disorder. 


\section{Methods}

\subsection{Overview}

In Section 2.2, we reviewed the author-topic model and how it could be applied to coordinate-based meta-analysis (Yeo et al. 2015). Section 2.3 discussed simulations and comparisons with spatial independent component analysis. Finally, the model was utilized in two different applications. In the first application (Section 2.4), we applied the author-topic model to discover cognitive components subserving self-generated thought. In the second application (Section 2.5), we estimated the co-activation patterns of the left IFJ.

\subsection{Author-topic model}

\subsubsection{Intuition behind the model}

The author-topic model was originally developed to discover topics from a corpus of text documents (Rosen-Zvi et al., 2010). The model represents each text document as an unordered collection of words written by a group of authors. Each author is associated with a probability distribution over topics, and each topic is associated with a probability distribution over a dictionary of words. Given a corpus of text documents, there are algorithms to estimate the distribution of topics associated with each author and the distribution of words associated with each topic. A topic is in some sense abstract, but is made concrete by its association with certain words and its association with certain authors. For example, if the author-topic model was applied to neuroimaging research articles, the algorithm might yield a topic associated with the author "Stephen Smith" and words like "fMRI", "resting-state" and "ICA". One might then interpret the topic posthoc as a "resting-state fMRI" research topic.

In a previous study (Yeo et al., 2015), the author-topic model was applied to neuroimaging meta-analysis (Figure 2) by treating task contrasts in the BrainMap database (Fox and Lancaster, 2002) as text documents, 83 BrainMap task categories (e.g., n-back) as authors, cognitive components as topics, and activation foci as words in the documents. Thus, the model encodes the premise that different behavioral tasks recruit multiple cognitive components, supported by overlapping brain regions.

Suppose a study utilizes one or more task categories, resulting in an experimental contrast yielding a collection of activation foci. Under the author-topic model, each activation focus is assumed to be generated by first randomly selecting a task from the set of tasks utilized in the experiment. Given the task, a component is randomly chosen based on the probability of a task recruiting a component $(\operatorname{Pr}($ component $\mid$ task $))$. Given the component, the location of the activation focus is then randomly chosen based on the probability that the component would 
activate a voxel $(\operatorname{Pr}($ voxel $\mid$ component $))$. The entire collections of $\operatorname{Pr}($ component $\mid$ task $)$ and $\operatorname{Pr}($ voxel $\mid$ component) are denoted as matrices $\theta$ and $\beta$, respectively. For example, the 2 nd row and 3rd column of $\theta$ corresponds to $\operatorname{Pr}(3$ rd component $\mid 2$ nd task) and the 4th row and 28th column of $\beta$ corresponds to $\operatorname{Pr}(28$ th voxel $\mid$ 4th component). Therefore, each row of $\theta$ and $\beta$ sums to 1. The formal mathematical definition of the model is provided in Supplemental Method S1.

A key property of the author-topic model is that the ordering of words within a document is exchangeable. When applied to meta-analysis, the corresponding assumption is that the ordering of activation foci is arbitrary. Although the ordering of words within a document is obviously important, the ordering of activation foci is not. For example, in the context of text documents, "dog has a bone" has a different meaning from "bone has a dog". On the other hand, in the context of a fMRI experiment, reporting parietal activation coordinates followed by prefrontal activation coordinates is equivalent to reporting prefrontal activation coordinates followed by parietal activation coordinates. Therefore, the author-topic model is arguably more suitable for meta-analysis than topic discovery from documents.

\subsubsection{Estimating the model parameters}

Given a collection of experiments with their associated activation coordinates and task categories, as well as the number of cognitive components $K$, the probabilities $\theta$ and $\beta$ can be estimated using various algorithms (Rosen-Zvi et al. 2010; Yeo et al., 2015; Ngo et al., 2016). Here, we chose to utilize the CVB algorithm because the algorithm was more robust to the choice of hyperparameters in smaller datasets. Although the CVB algorithm for the authortopic model was first introduced in a conference article (Ngo et al., 2016), detailed derivations have not been published. For completeness, detailed derivations of the author-topic CVB algorithm are provided in Supplemental Method S2. Explanations of why the CVB algorithm is theoretically better than the EM algorithm and standard variational Bayes inference are found in Supplemental Method S3. In this work, Bayesian information criterion (BIC) was used to estimate the optimal number of cognitive components (Supplemental Method S4). Further implementation details are found in Supplemental Method S5.

\subsubsection{Input to the author-topic model}

Each task activation contrast was associated with a set of activation foci. The spatial locations (i.e., coordinates) of the activation foci were reported in or transformed to the 
MNI152 coordinate system (Lancaster et al., 2007). Using standard meta-analysis procedure (Wager et al., 2009; Yarkoni et al. 2011; Yeo et al. 2015), a 2mm-resolution binary activation image was created for each experimental contrast, in which a voxel was given a value of 1 if it was within a $10 \mathrm{~mm}$-radius of any activation focus, and 0 otherwise. Thus, the set activated voxels of each experiment in the author-topic model corresponds to the set of voxels with a value of 1 in the corresponding $2 \mathrm{~mm}$-resolution binary activation image. We note that the exact choice of smoothing radius did not significantly affect the results (see Section 3.4).

\subsection{Simulations}

\subsubsection{Independent component analysis (ICA)}

ICA is a data-driven technique that has been widely applied to fMRI (Calhoun et al. 2001; Beckmann and Smith, 2004). ICA has also been successfully applied to coordinate-based meta-analysis (Smith et al., 2009). However, the author-topic model has a few significant advantages over ICA in the case of coordinate-based meta-analysis. First, activation foci are binary data in the sense that a voxel is either reported to be activated or not in an experiment. However, ICA requires positive and negative values in the input data, which involves demeaning the binary values at each voxel (across experiments). In contrast, the author-topic model makes direct use of the binary activation data. Second, the author-topic model is able to exploit task categorical information (red task layer in Figure 2), which is non-trivial to introduce in ICA.

Most importantly, ICA estimates can be negative, which do not make sense in the case of coordinate-based meta-analysis. For example, a task should not be allowed to be negatively associated with a component, since task activation and de-activation in a coordinate-based meta-analysis are typically handled separately. Similarly, it does not make sense for the activation maps associated with each component to be negative. The situation is of course reversed in image-based meta-analysis (Salimi-Khorshidi et al., 2009), where there might be both activation and de-activation. For image-based meta-analysis, it does make sense to talk about components being negatively recruited by a task and ICA makes more theoretical sense than the author-topic model.

\subsubsection{Simulation details}

Here, we considered simulations to compare the effectiveness of the author-topic model and ICA. More specifically, we considered a hypothetical situation in which five tasks from a functional domain recruited two cognitive components with different probabilities (Figure 3A). 
The two components have distinct activation patterns on a 2D "brain" of 256 by 256 pixels. More specifically, each component is associated with activations within two Gaussian distributions centered at two opposite quadrants of the 2D brain (Figure 3B). Given the activation foci of multiple experiments (task contrasts), the goal was to automatically recover the two cognitive components using either the author-topic model or ICA.

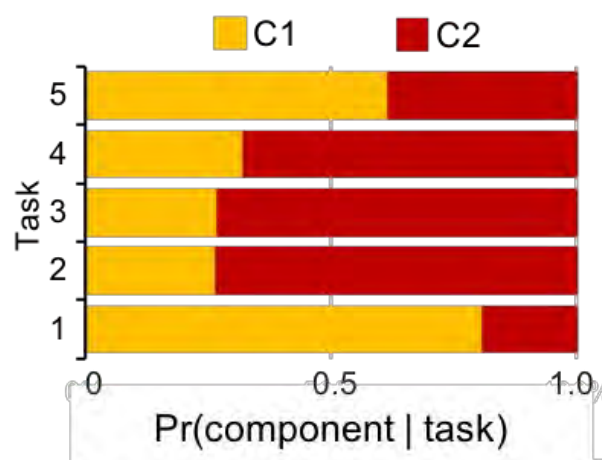

A. Probability that a task
would recruit a component

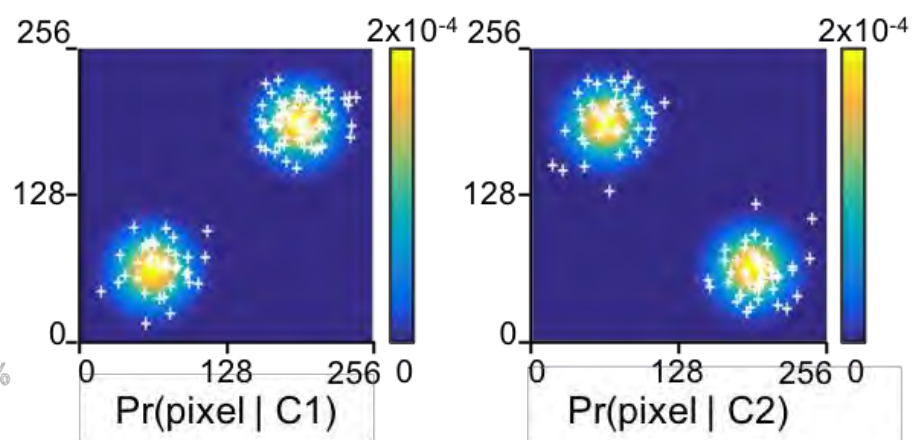

B. Activation patterns of two components

Figure 3. Simulation of heterogeneity in coordinate-based meta-analysis. (A) Bar chart shows five tasks from a functional domain recruiting two cognitive components with different probabilities. (B) Activation patterns of two components on a 2D "brain" of 256 by 256 pixels. Each component is associated with activations (white crosses) within two Gaussian distributions centered at two opposite quadrants of the 2D "brain". For each simulation run, the probability of a task recruiting a component and the covariances of each component's $2 \mathrm{D}$ Gaussian distributions were randomly generated. The author-topic model and ICA were then applied to recover the two components. We note that ICA mixture weights can be negative, which does not make sense in the context of coordinate-based meta-analysis. As such, we discarded simulation runs if any of the ICA estimates yielded negative weights.

A single simulation run comprised 150 experiments (task contrasts), which is comparable to a typical meta-analysis (c.f. self-generated thought in Section 2.4). Each experiment (task contrast) was randomly assigned to one of the five tasks, with the contrast distributions skewed towards two of the five tasks to simulate the fact that some tasks are more popular than others in the literature. Furthermore, each task contrast is randomly chosen to have between 1 to 10 activation foci. For each activation focus, a component was randomly sampled based on the probability of components given the task assigned to the experiment. For the given component, one of the 2-D Gaussian distributions of each component was randomly chosen with equal probabilities (Figure 3B). The spatial location of the activation focus was then randomly sampled from the Gaussian distribution. The activation focus was smoothed with a binary smoothing kernel, such that all pixels within 10 voxels from an activation focus were given a value of 1 , and 0 otherwise. 
For a given simulation run, the latent components were estimated using either ICA or the author-topic model. We considered three possible ICA setups. The first two setups (ICA1 and ICA2) utilized CanICA (Varoquaux et al., 2010), an ICA decomposition implementation provided with Nilearn (Abraham et al. 2014). CanICA extracts representative patterns of multisubject fMRI data by performing ICA on a data subspace common to the group (Varoquaux et al., 2010). In the two setups ICA1 and ICA2, each task was treated as a subject. In ICA1, the activation maps of all experiments assigned to the same task were summed together, i.e., each task was treated as a single subject with a single time point. In ICA2, each task was treated as a single subject, but the experiments assigned to the given task were treated as separate time points of the subject. The third setup (ICA3) utilized the MELODIC implementation of ICA from the FSL package (Beckmann \& Smith 2004; Smith et al. 2004).

To evaluate the estimation quality, Pearson's correlation coefficient was computed between the groundtruth probability distribution of a component activating a vertex $(\operatorname{Pr}($ vertex ( component)) against the estimates from the author-topic model or ICA. Pearson's correlation coefficient was also computed between the groundtruth distribution of components given a task $(\operatorname{Pr}($ component $\mid$ task $))$ and estimates from the author-topic model or ICA.

The simulation was repeated multiple times. For a given simulation run, the covariances of each component's 2D Gaussian distributions were randomly generated (Figure 3B). The probability of a task recruiting a component was also randomly generated (Figure 3A). As explained previously (Section 2.3.1), ICA's mixture weights can be negative, which implies negative associations between tasks and components. This does not make sense in the case of coordinate-based meta-analysis, so we discarded simulation runs if any of the ICA estimates yielded negative weights. Overall, we ran roughly 300 simulation runs in order to yield exactly 100 simulation runs, in which ICA estimates were valid.

\subsection{Self-generated thought}

\subsubsection{Activation foci of experiments involving self-generated thought}

To explore cognitive components subserving self-generated thought, we considered 1812 activation foci from 179 experimental contrasts across 167 imaging studies, each employing one of seven task categories subjected to prior meta-analysis with GingerALE (Fox and Lancaster, 2002; Laird et al., 2009, 2011; Fox et al., 2014; http://brainmap.org/ale). Of the 167 studies, 48 studies employed "Autobiographical Memory" $(\mathrm{N}=19)$, "Navigation" $(\mathrm{N}=$ 13) or "Task Deactivation" $(\mathrm{N}=16)$ tasks. The 48 studies were employed in a previous metaanalysis examining the default network (Spreng et al., 2009). There were 79 studies involving 
"Story-based Theory of Mind" ( $\mathrm{N}=18)$, "Nonstory-based Theory of Mind" $(\mathrm{N}=42)$ and "Narrative Comprehension" $(\mathrm{N}=19)$ tasks. The 79 studies were utilized in a previous metaanalysis examining social cognition and story comprehension (Mar, 2011). Finally, there were 40 studies involving the "Moral Cognition" task that was again utilized in a previous metaanalysis (Sevinc and Spreng, 2014). The list of all experiments included in the dataset are provided in Supplemental Method S7. The criteria for selecting the experiments can be found in the original meta-analyses (Spreng et al., 2009; Mar, 2011; Sevinc and Spreng, 2014). All foci coordinates were in or transformed to the MNI152 coordinate system (Lancaster et al., 2007).

\subsubsection{Discovering cognitive components of self-generated thought}

The application of the author-topic model to discover cognitive components subserving self-generated thought (Figure 2) is conceptually similar to the original application to the BrainMap (Yeo et al., 2015). The key difference is that the current application is restricted to seven related tasks in order to discover heterogeneity within a single functional domain, while the original application sought to find common and distinct cognitive components across domains.

The model parameters are the probability of a task recruiting a component $(\operatorname{Pr}($ component $\mid$ task $))$ and the probability of a component activating a brain voxel (Pr(voxel | component)). The parameters were estimated from the 1812 activation foci from the previous section using the CVB algorithm (Supplemental Methods S2 and S5). BIC was used to estimate the optimal number of cognitive components (Supplemental Method S4).

\subsubsection{Interpreting cognitive components of self-generated thought}

We note that $\operatorname{Pr}$ (component $\mid$ task) can be interpreted as follows. Suppose $\operatorname{Pr}$ (component $\mathrm{C} 1$ | autobiographical memory) is equal to 0.63 and we have an autobiographical memory experiment that reported 100 activation foci. Then, on average, 63 foci will fall inside component $\mathrm{C} 1$.

The matrix $\operatorname{Pr}($ voxel $\mid$ component), $\beta$, can be interpreted as $K$ brain images in MNI152 coordinate system (Lancaster et al., 2007), where $K$ is the number of cognitive components. Volumetric slices highlighting specific subcortical structures were displayed using FreeSurfer (Fischl, 2012). The cerebral cortex was visualized by transforming the volumetric images from MNI152 space to fs_LR surface space using Connetome Workbench (Van Essen et al., 2013) via the FreeSurfer surface space (Buckner et al., 2011; Fischl et al., 2012). For visualization 
purpose, isolated surface clusters with less than 20 vertices were removed. $\operatorname{Pr}$ (component $\mid$ task) was thresholded at $1 / \mathrm{K}$, and $\operatorname{Pr}$ (voxel | component) was thresholded at 1e-5, consistent with previous work (Yeo et al., 2015). Unthresholded maps of the components are available on NeuroVault (Gorgolewski et al., 2015) at https://neurovault.org/collections/4684/.

\subsubsection{Goodness offit}

For each task, we computed the weighted average of the cognitive components (Pr(voxel | component)), where the weights corresponded to the probabilities of the task recruiting the components $(\operatorname{Pr}($ component $\mid$ task)). This weighted average spatial map could be interpreted as the model estimate of the "ideal" (reconstructed) activation map for a particular task. The model fit was good if a task's reconstructed activation map was similar to the empirical activation map of the task (obtained by averaging the activation maps of all experiments employing the task). Therefore, we computed Pearson's correlation coefficient between all pairs of reconstructed and empirical activation maps, yielding a 7 x 7 correlation matrix (since there were 7 tasks).

\subsubsection{Correspondence between cognitive components and resting-state networks}

Motivated by similarities between task and resting-state networks (Smith et al., 2009; Laird et al., 2011; Yeo et al., 2015), we compared the cognitive components of self-generated thought with a previously published set of 17 resting-state networks (Yeo et al., 2011). For each resting-state network and each cognitive component, the probability of the cognitive component activating a voxel $(\operatorname{Pr}(\operatorname{voxel} \mid$ component)) was averaged across all voxels within the network, resulting in an average probability of a component activating the given network.

\subsection{Left inferior frontal junction (IFJ)}

\subsubsection{Activation foci of experiments activating the left IFJ}

To explore task-dependent co-activation patterns expressed by the left IFJ, we considered activation foci from experiments reporting activation within a left IFJ seed region (Figure S1) delineated by a previous study (Muhle-Karbe et al. 2015). Muhle-Karbe and colleagues performed a co-activation-based parcellation of a left lateral prefrontal region into six parcels, including an IFJ region (Muhle-Karbe et al., 2015). The parcellation procedure assumed that voxels within a parcel exhibited a single co-activation pattern. Thus, the advantage of using this particular IFJ seed region (instead of an IFJ region from a different 
study) is that this region is thought to exhibit a single co-activation pattern (according to MACM).

This seed region is publicly available on ANIMA (Reid et al. 2016; http://anima.fzjuelich.de/studies/MuhleKarbe_2015 IFJ). We selected experiments from the BrainMap database with at least one activation focus falling within the IFJ seed region. We further restricted our analyses to experimental contrasts involving normal subjects. Overall, there were 323 experiment contrasts from 238 studies with a total of 5201 activation foci. The list of all experiments included in the dataset are provided in Supplemental Method S8.

\subsubsection{Discovering co-activation patterns of the IFJ}

To apply the author-topic model to discover co-activation patterns, we consider each of the 323 experimental contrasts to employ its own unique task category (Figure 4). In the parlance of the author-topic model, we assumed each document (experiment) has its own unique author (task). The premise of the model is that the IFJ expresses one or more overlapping co-activation patterns depending on task contexts. A single experiment activating the IFJ might recruit one or more co-activation patterns. The model parameters are the probability that an experiment would recruit a co-activation pattern $(\operatorname{Pr}$ (co-activation pattern experiment)), and the probability that a voxel would be involved in a co-activation pattern $(\operatorname{Pr}($ voxel $\mid$ co-activation pattern $))$. The parameters were estimated from the 5201 activation foci from the previous section using the CVB algorithm (Supplemental Method S2 and S5). BIC was used to estimate the optimal number of co-activation patterns (Supplemental Method S4). 


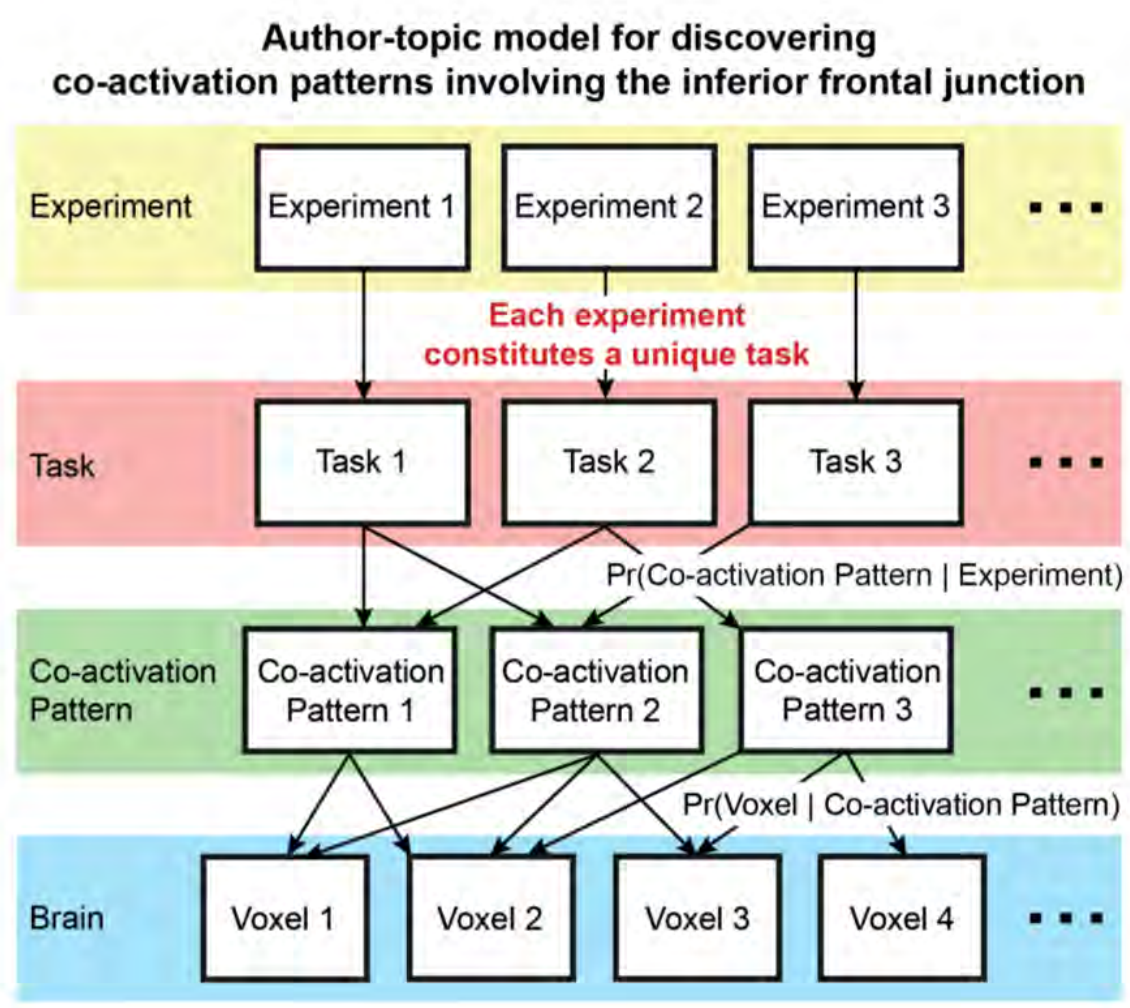

Figure 4. Author-topic model for discovering co-activation patterns of the inferior frontal junction (IFJ). In contrast to Figure 2, this instantiation of the model assumes that each experiment constitutes a unique task. The premise of the model is that the IFJ expresses multiple overlapping task-dependent co-activation patterns. The model parameters are the probability of an experiment recruiting a co-activation pattern $(\operatorname{Pr}($ co-activation pattern experiment)), and the probability of a voxel being associated with a co-activation pattern (Pr(voxel | co-activation pattern)).

\subsubsection{Interpreting co-activation patterns of the IFJ}

Similar to the previous application on self-generated thought, the matrix $\operatorname{Pr}($ voxel $\mid$ coactivation pattern), $\beta$, was visualized as $K$ brain images in both fsLR surface space and MNI152 volumetric space. Like before, isolated surface clusters with less than 20 vertices were removed for the purpose of visualization. Unthresholded spatial maps of the co-activation patterns are available on NeuroVault (Gorgolewski et al., 2015) at https://neurovault.org/collections/4718/.

Because each of the 323 experiments was treated as employing a unique task category, $\operatorname{Pr}($ co-activation pattern $\mid$ experiment), $\theta$, is a matrix of size $K \times 323$. $\theta$ was further mapped onto BrainMap task categories to assist in the interpretation. More specifically, since the experiments were extracted from the BrainMap database, each experiment was tagged with one or more BrainMap task categories (Table S1). The Pr(co-activation pattern | experiment) was averaged across experiments employing the same task category to estimate the probability that 
a task category would recruit a co-activation pattern $(\operatorname{Pr}($ co-activation pattern $\mathrm{c} \mid$ task $\mathrm{t}))$. Further details of this procedure are found in Supplemental Method S6. The Pr(co-activation pattern $\mid$ task) can be interpreted as follows. Suppose $\operatorname{Pr}$ (co-activation pattern C1 | semantic monitoring/discrimination) is equal to 0.51 and we have a semantic monitoring/discrimination experiment that reports activation in left IFJ and 100 activation foci. Then, on average, 51 foci will fall inside co-activation pattern $\mathrm{C} 1$.

We note that directly using the BrainMap task categories to interpret the co-activation patterns is tricky. This is because a BrainMap task category might only have a very small percentage of experiments activating the IFJ, so these experiments might not be representative of the task category. For example, of the 230 experiments in the BrainMap database labeled as the "Encoding" task category, only 13 experiments reported activations in the left IFJ. Thus, the 13 experiments were not simply encoding tasks, but encoding tasks that happened to activate the IFJ. This is the reason why the BrainMap task categories were not directly utilized in the author-topic model for the IFJ analysis and that each experiment was treated as employing a unique task category (c.f. self-generated thought in Section 2.4).

To ensure an appropriate interpretation, we inspected the original publications associated with the top three experiments with the highest $\operatorname{Pr}($ co-activation pattern experiment) for each of the top three tasks associated with each co-activation pattern, i.e., nine publications for each co-activation pattern. The literature analysis allowed us to determine if there were common neural processes underlying the subset of experiments within each task category that strongly activated the IFJ.

\subsubsection{Goodness of fit}

For each co-activation pattern, activation maps of the top three experiments with the highest probability of recruiting a co-activation pattern (i.e., $\operatorname{Pr}$ (co-activation pattern | experiment)) for each of the top three tasks associated with the co-activation pattern (i.e., nine activation maps in total) were averaged, resulting in an empirical activation map associated with each co-activation pattern. The model fit was good if the empirical activation map was similar to the estimated co-activated pattern. Therefore, we computed Pearson's correlation coefficient between all pairs of empirical activation maps and co-activation maps, yielding a $K \times K$ correlation matrix, where $\mathrm{K}$ is the number of co-activation patterns estimated by BIC. 


\subsection{Data and code availability}

Activation foci from the meta-analysis of self-generated thought and the source code of the author-topic model, including the visualization and analysis tools, are publicly available at

https://github.com/ThomasYeoLab/CBIG/tree/master/stable_projects/metaanalysis/Ngo2019 AuthorTopic. The activation foci from the meta-analysis of IFJ can be obtained via a collaborative-use license agreement with BrainMap (http://www.brainmap.org/collaborations.html). 


\section{$3 \quad$ Results}

\subsection{Overview}

In Section 3.2, we show simulation results suggesting that the author-topic model compares favorably with ICA in the goal of discovering latent patterns in coordinate-based meta-analysis. We then explored the cognitive components of self-generated thought (Section 3.3) and the co-activation patterns of the IFJ (Section 3.4). Finally, Section 3.5 discusses a few control analyses.

\subsection{Simulations}

Figure 5 shows the results of one representative simulation (see Section 2.3 for details). Figure 5A shows the groundtruth 2D "brain" maps for this representative simulation run. The two leftmost columns show simulated activation foci as white crosses overlaid on top of the 2D Gaussian distributions used to generate the foci. The rightmost bar chart shows the probability of each of the 5 tasks recruiting a component.

The rightmost column of Figure 5B shows the author-topic model estimates of the probability of each of the 5 tasks recruiting a component. The rightmost column of Figures $5 \mathrm{C}$ to $5 \mathrm{E}$ shows the ICA mixture weights, normalized so they sum to one ${ }^{2}$. The mixture weights represent the association between the tasks and the components. The numbers at the bottom of each panel are the correlations between the estimates and groundtruth averaged across 100 simulation runs. In general, the author-topic model yielded better estimates of the associations between tasks and components.

Figure 5B shows the author-topic model estimates, while Figures $5 \mathrm{C}$ to $5 \mathrm{E}$ show the ICA estimates. The two leftmost columns show the spatial maps of the two components estimated by the author-topic model or ICA. The numbers at the bottom of each panel are the correlations between the estimated and groundtruth "brain" maps averaged across 100 simulation runs. In general, the author-topic model yielded better estimates of the groundtruth "brain" maps. It is also worth noting that the ICA spatial maps showed negative values, even though the simulation runs had been constrained to those where ICA mixture weights were positive $^{3}$. As previously explained (Section 2.3.1), negative values are not meaningful in the context of coordinate-based meta-analysis.

\footnotetext{
2 Recall that simulation runs were discarded if ICA yielded negative weights.

${ }^{3}$ Note that this is after adding back the mean signal removed during the ICA de-meaning step.
} 

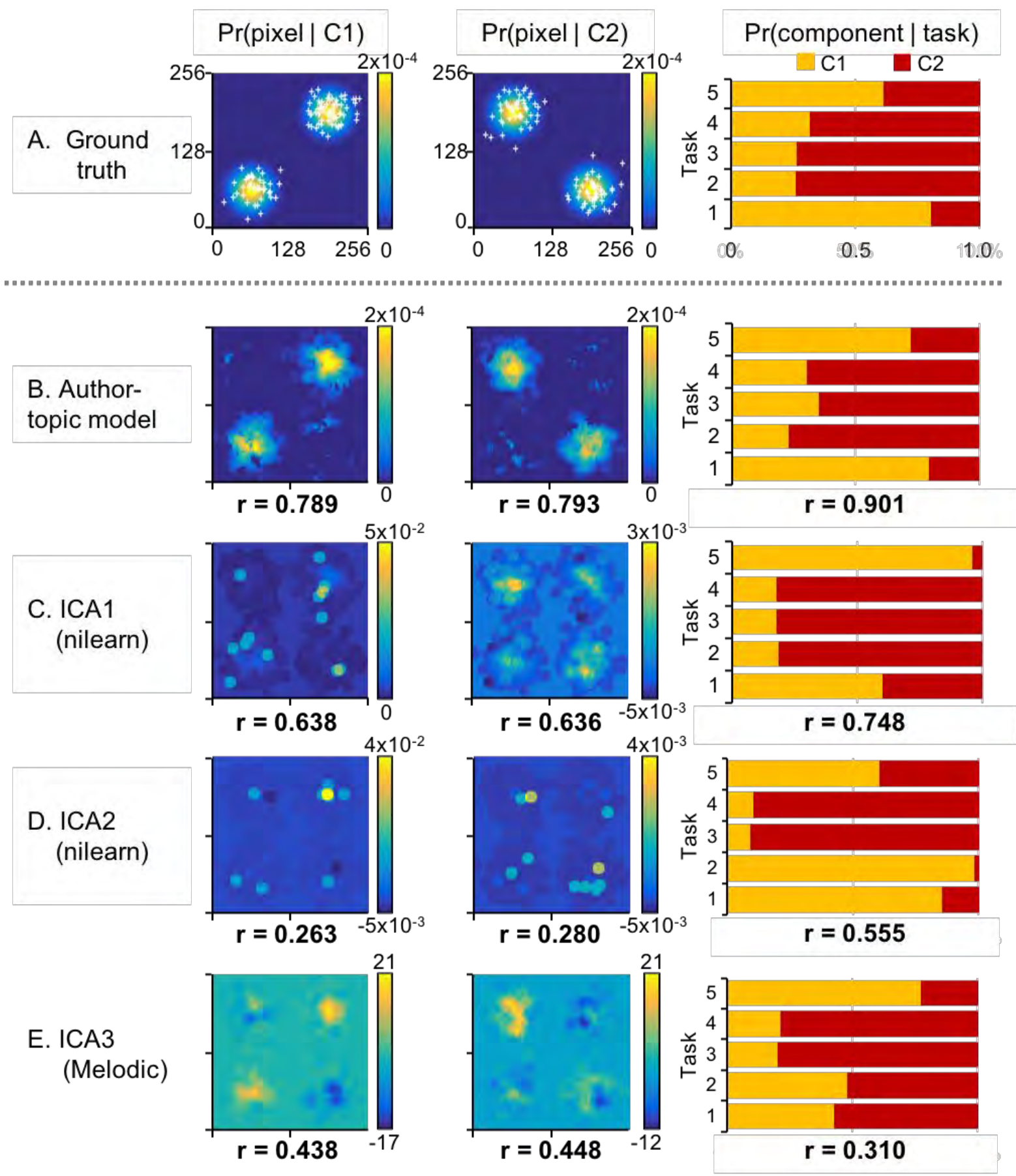

Figure 5. Simulation comparing the author-topic model and ICA. (A) Single representative simulation run. Two leftmost columns show activation foci (white crosses) on top of Gaussian distributions used to generate the foci. Rightmost bar chart shows the probability of each of the 5 tasks recruiting a component. (B) Author-topic model estimates. (C) ICA estimates. Number below each panel is the correlation between model estimates and groundtruth averaged across 100 simulation runs. Observe that ICA can yield negative weights, which do not make sense in the context of a coordinate-based meta-analysis (see discussion in Section 2.3.1). We note that about 300 simulation runs were run in order to generate 100 simulation runs in which ICA estimates of mixture weights were non-negative. 


\subsection{Self-generated thought}

\subsubsection{ALE meta-analysis of self-generated thought}

Figure 6 shows the activation likelihood estimate (ALE) of experiments involving selfgenerated thought. Statistical significance was established with 1000 permutations. The map was thresholded at a voxel-wise uncorrected threshold of $\mathrm{p}<0.001$ and cluster-level familywise error rate threshold of $\mathrm{p}<0.01$. Consistent with previous studies, ALE reveals a constellation of regions typically referred to as the default network (Raichle et al., 2001; Buckner et al. 2008; Spreng et al. 2009). However, as previously discussed, ALE cannot reveal functional sub-domains within self-generated thought without prior assumptions about the subdomains. Therefore, in the next section, we explored the use of the author-topic model.

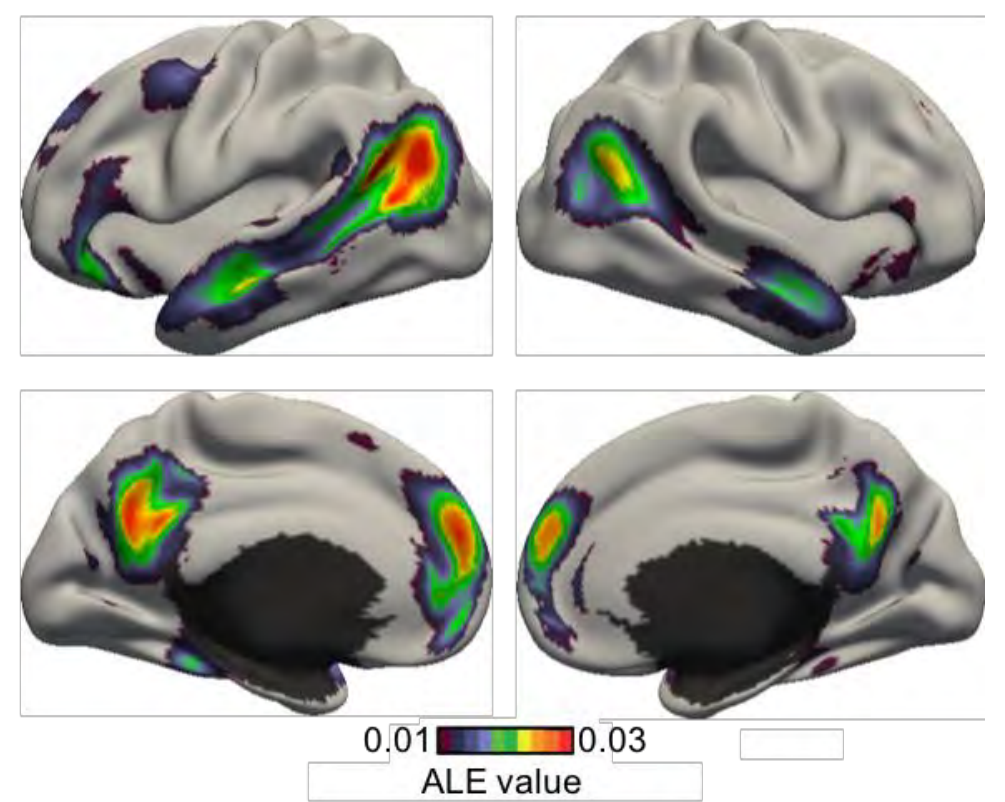

Figure 6. Activation likelihood estimate (ALE) of experiments involving self-generated thought. Consistent with previous studies, ALE reveals a set of regions corresponding to the default network. However, ALE cannot provide insights into functional sub-domains without prior assumptions about the sub-domains.

\subsubsection{Cognitive components of self-generated thought}

Figure 7 shows the cognitive components of self-generated thought estimated by the author-topic model. Figure 7A shows the BIC score as a function of the number of estimated cognitive components. A higher BIC score indicates a better model. Because the 2-component estimate achieved the highest BIC score, subsequent results will focus on the 2 -component estimate. 
The 2-component estimate is shown in Figure 7B. The seven tasks recruited the two cognitive components to different degrees. The top tasks recruiting component $\mathrm{C} 1$ were "Navigation" and "Autobiographical Memory". In contrast, the top tasks recruiting component C2 were "Narrative Comprehension", "Theory of Mind (story-based)", "Task deactivation”, "Theory of Mind (nonstory-based)", and "Moral Cognition".

\section{A. Bayesian Information Criterion (BIC)}

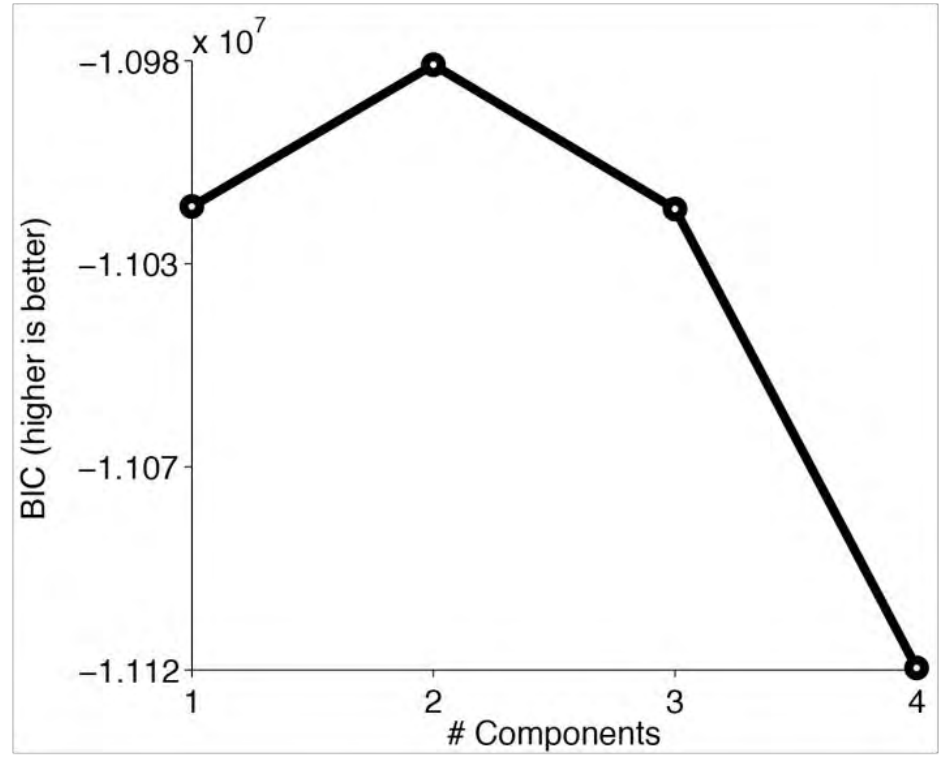

\section{B. Cognitive components of self-generated thought}

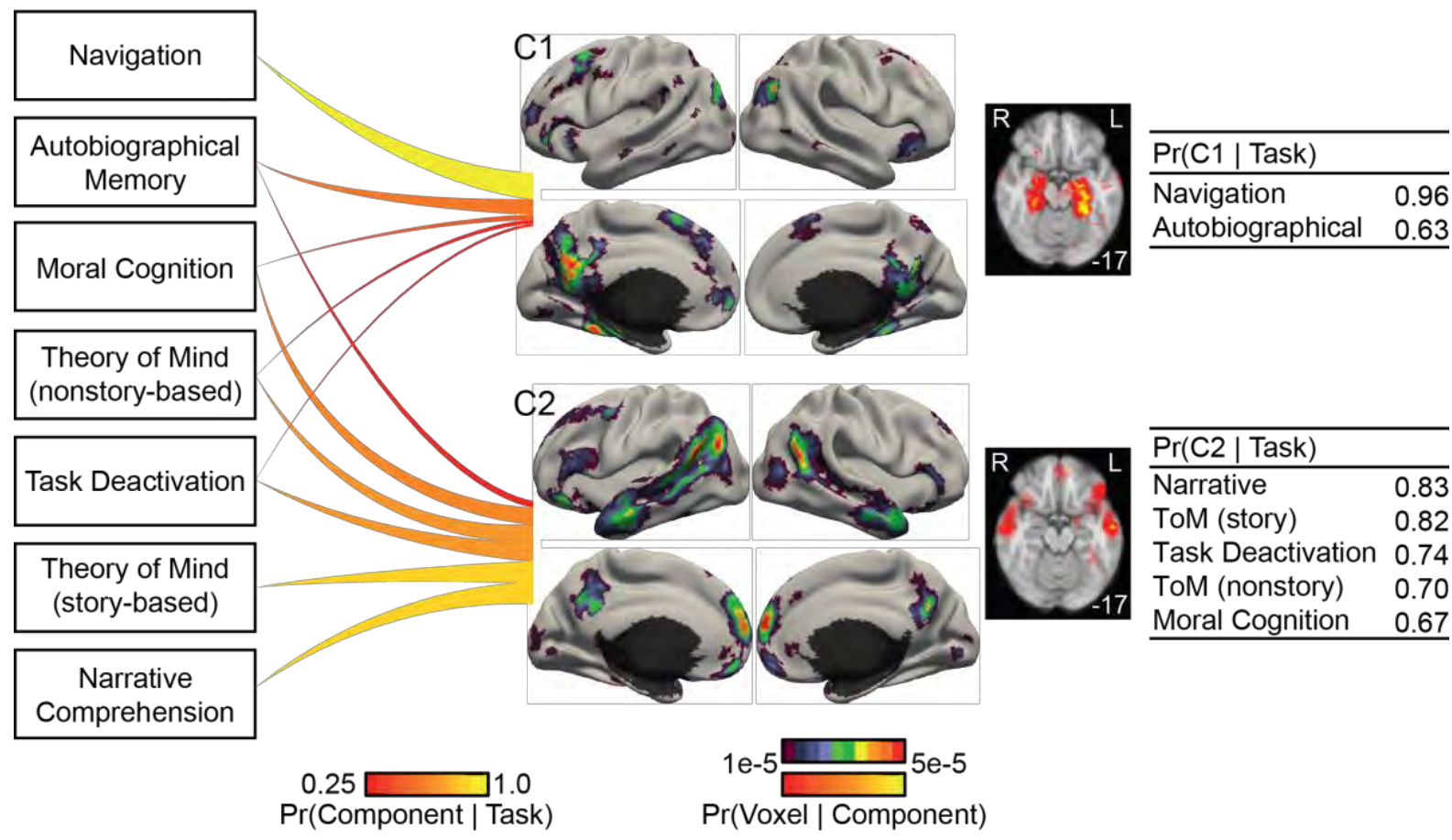

Figure 7. Cognitive components of self-generated thoughts. (A) Bayesian Information Criterion (BIC) plotted as a function of the number of estimated cognitive components. A higher BIC indicates a better model. BIC peaks at 2 components. (B) 2-component model 
estimates. Each line connects 1 task with 1 component. The thickness and brightness of the lines are proportional to the magnitude of $\operatorname{Pr}($ component $\mid$ task). For each component, the four leftmost figures show the surface-based visualization for the probability of components activating different brain voxels (i.e., $\operatorname{Pr}($ voxel $\mid$ component)), whereas the rightmost figure show a volumetric slice highlighting subcortical structures being activated differently across components. The top color bar is utilized for the surface-based visualization, whereas the bottom color bar is utilized for the volumetric slices. The tables on the right show the top tasks most likely to recruit the two components. The numbers in the right column correspond to $\mathrm{Pr}$ (component | task). Navigation and Autobiographical Memory preferentially recruited component C1, whereas Narrative Comprehension, Theory of Mind (ToM), Task Deactivation and Moral Cognition preferentially recruited component C2.

Compared with Figure 6, the two cognitive components appeared to decompose the activation pattern revealed by ALE. The two cognitive components appeared to activate different portions of the default network (Figure 7B). Focusing our attention to the medial cortex, both components had high probability of activating the medial parietal cortex. However, while component C2's activation was largely limited to the precuneus, component C1's activation also included the posterior cingulate and retrosplenial cortices in addition to the precuneus. Both components also had high probability of activating the medial prefrontal cortex (MPFC). However, component C1's activations were restricted to the middle portion of the MPFC, while component C2's activations were restricted to the dorsal and ventral portions of the MPFC. Finally, component $\mathrm{C} 1$, but not component $\mathrm{C} 2$, had high probability of activating the hippocampal complex.

Switching our attention to the lateral cortex, component $\mathrm{C} 1$ had high probability of activating the posterior inferior parietal cortex, while component $\mathrm{C} 2$ had high probability of activating the entire stretch of cortex from the temporo-parietal junction to the temporal pole. Component $\mathrm{C} 2$ was significantly more likely than component $\mathrm{C} 1$ to activate the inferior frontal gyrus.

\subsubsection{Goodness of fit}

Figure 8 shows the correlation matrix between the empirical activation maps of seven tasks involving self-generated thought (rows) and seven task activation maps reconstructed from the author-topic model parameter estimates (columns). The diagonal entries of the correlation matrix were significantly higher than the off-diagonal entries: average diagonal entry was 0.69 , while the average off-diagonal entry was 0.50 . Overall, this suggests a good model fit. However, the diagonal entries were not always the highest and there was a clear block-diagonal structure. Not surprisingly, the top left block corresponded to the top two tasks 
recruiting component $\mathrm{C} 1$ (Figure 7), while the bottom right block corresponded to the top five tasks recruiting component C2 (Figure 7).

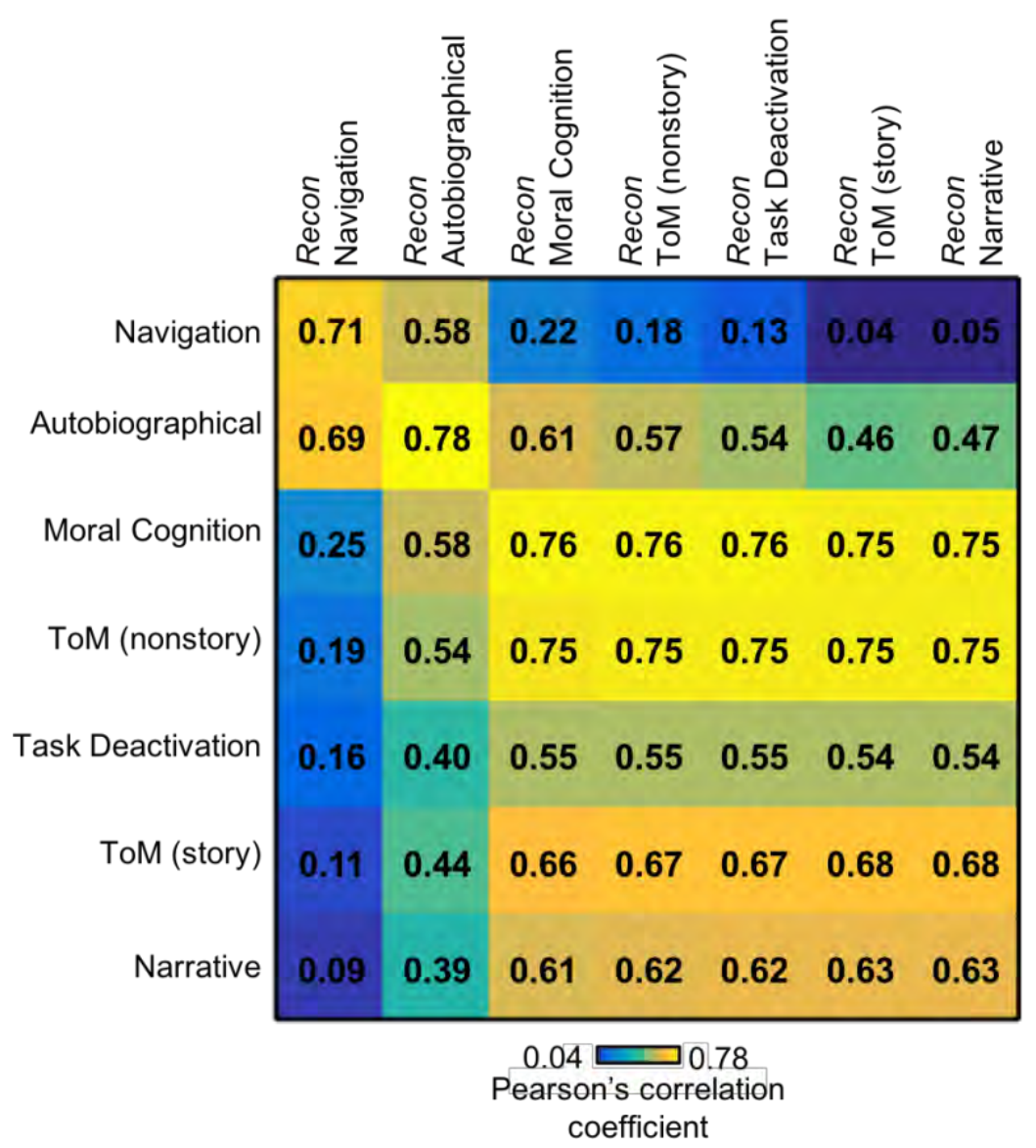

Figure 8. Goodness of fit of the author-topic model for self-generated thought. The matrix represents the correlations between the empirical activation maps (rows) and reconstructed activation maps (columns) of seven tasks. The tasks follow the same ordering as in Figure 7. The diagonal values (average $r=0.69$ ) were larger than off-diagonal values (average $r=0.50$ ), suggesting a good model fit.

\subsubsection{Correspondence with resting-state networks}

The average probability of each self-generated thought cognitive component activating each resting-state network (Yeo et al., 2011) is shown in Figure S2. Four resting-state networks with the highest probability of being activated by either component are shown in Figure 9. Three of these resting-state networks were previously considered to be fractionation of the default network (Yeo et al., 2014).

The Default $\mathrm{C}$ resting-state network was most strongly activated by component $\mathrm{C} 1$, while the temporal parietal resting-state network was most strongly activated by component 
$\mathrm{C} 2$. On the other hand, Default A and B resting-state networks were preferentially activated by components $\mathrm{C} 2$.

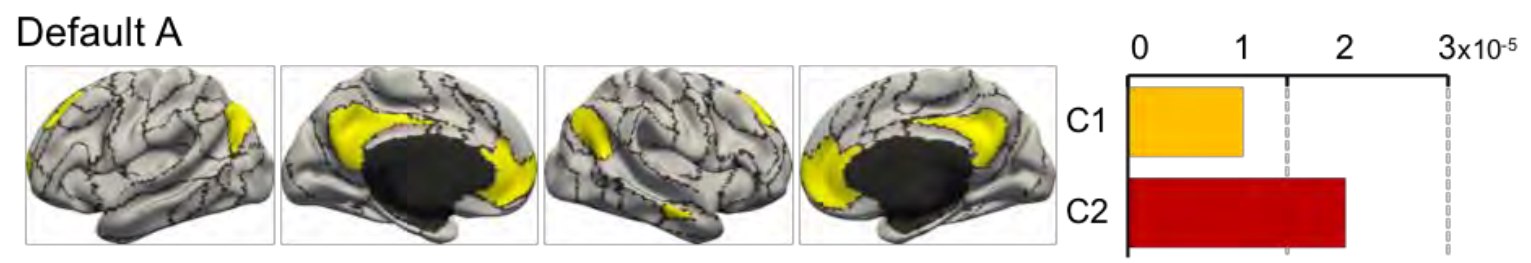

Default B
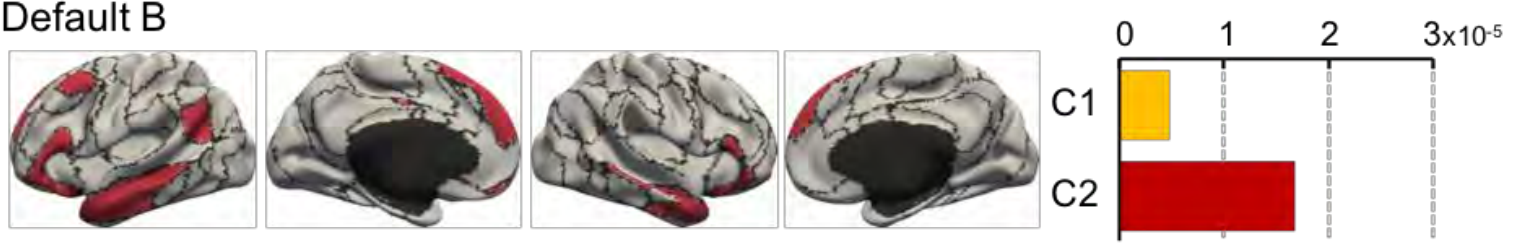

Default C
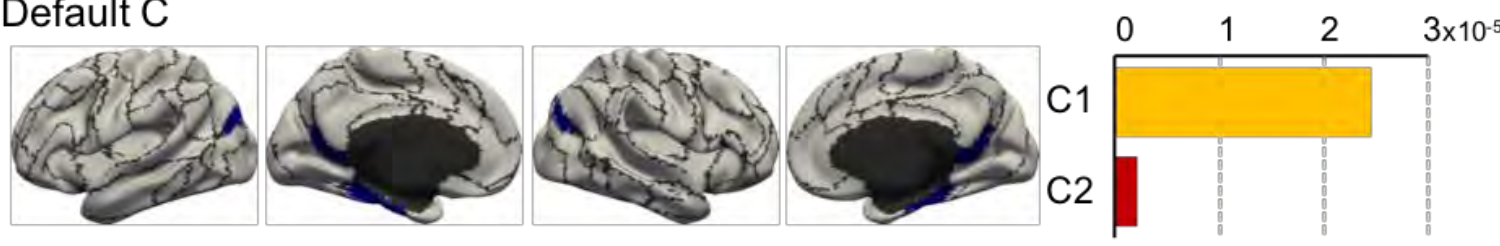

Temporal parietal
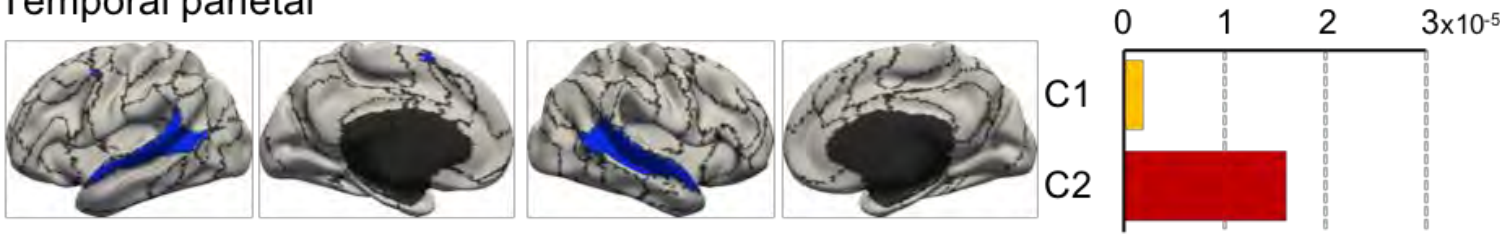

Figure 9. Average probability of self-generated thought cognitive components activating voxels within 4 resting-state networks (Yeo et al. 2011). The naming of the four resting-state networks followed the convention of previous literature (Kong et al., 2018; Li et al., 2018). Default $\mathrm{C}$ resting-state network was primarily activated by component $\mathrm{C} 1$, while the temporal parietal resting-state network was primarily activated by component $\mathrm{C} 2$. On the other hand, Default $\mathrm{A}$ and $\mathrm{B}$ resting-state networks were preferentially activated by component $\mathrm{C} 2$.

\subsection{Left inferior frontal junction (IFJ)}

\subsubsection{ALE meta-analysis of the left IFJ's co-activation pattern}

Figure 10 shows the co-activation pattern of the left IFJ estimated by the application of ALE to meta-analytic co-activation modeling (Muhle-Karbe et al., 2015). Statistical significance was established with 1000 permutations. The map was thresholded at a voxel-wise uncorrected threshold of $\mathrm{p}<0.001$ and cluster-level family-wise error rate threshold of $\mathrm{p}<$ 0.01. The co-activation pattern was mostly bilateral and involved dorsolateral prefrontal cortex, anterior insula, superior parietal lobules, posterior medial frontal cortex and the fusiform gyri. As previously discussed, ALE delineates regions consistently activated across studies, but 
cannot reveal potential task-dependent co-activation patterns. Therefore, in the next section, we explored the use of the author-topic model.

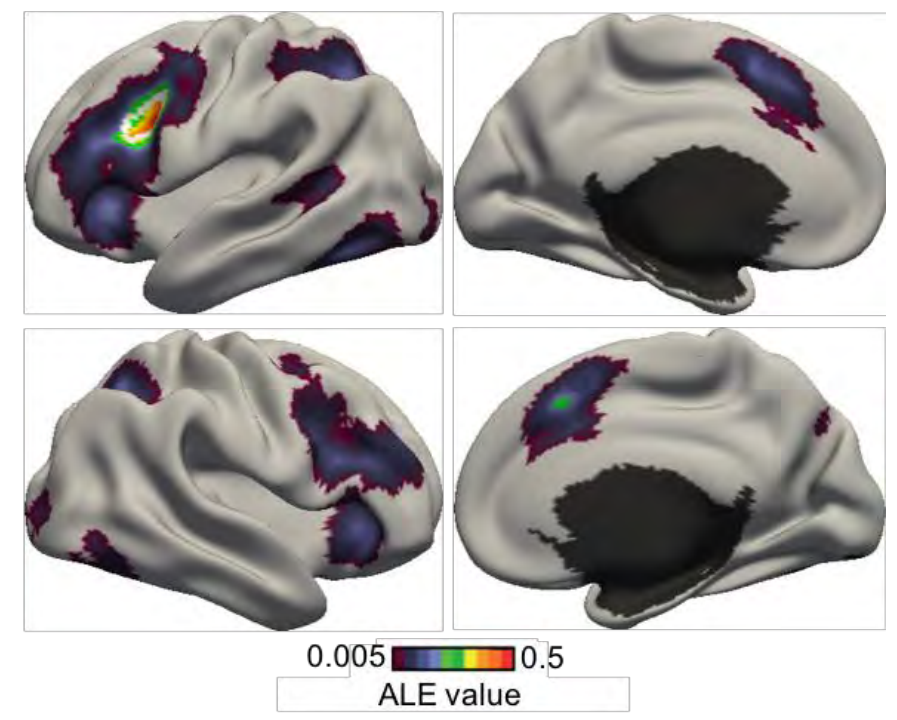

Figure 10. Co-activation pattern of the left inferior frontal junction (IFJ) estimated by the application of ALE to perform meta-analytic co-activation mapping. The IFJ seed region is delineated by a white boundary.

\subsubsection{Task-dependent co-activation patterns of the left IFJ}

Figure 11 shows the co-activation patterns of the left IFJ estimated by the author-topic model. Figure 11A shows the BIC score as a function of the number of estimated co-activation patterns. There were two peaks corresponding to the 3-pattern and 5-pattern estimates. Figure $\mathrm{S} 3$ shows the 5-pattern estimate. Although the 5-pattern estimate had a higher BIC score than the 3-pattern estimate, the co-activation patterns appeared to fractionate the IFJ into smaller territories. While this fractionation was intriguing, our goal was to examine if the IFJ exhibited task-dependent co-activation patterns and not whether it can be further fractionated. Thus, the 5-pattern estimate represented a degenerate solution from this perspective ${ }^{4}$.

Figure 11B shows the co-activation patterns from the 3-pattern estimate. Unlike the 5pattern estimate, the 3 co-activation patterns appeared to overlap completely within the IFJ. Therefore, subsequent results will focus on the 3-pattern estimate. Overall the 3 co-activation patterns appeared to decompose the consensus co-activation pattern revealed by ALE (Figure $10)$.

\footnotetext{
${ }^{4}$ Given that the "undesirable" 5-pattern estimate had the highest BIC, these results emphasized the fact that the BIC should only be treated as a guide to the number of cognitive components or co-activation patterns, rather than providing a definitive answer.
} 
Co-activation pattern $\mathrm{C} 1$ was left lateralized and might be recruited in tasks involving language processing. Co-activation pattern $\mathrm{C} 2$ involved bilateral superior parietal and posterior medial frontal cortices, and might be recruited in tasks involving attentional control. Coactivation pattern C3 involved bilateral frontal cortex, anterior insula and posterior medial frontal cortex, and might be recruited in tasks involving inhibition or response conflicts.

\section{A. Bayesian Information Criterion (BIC)}

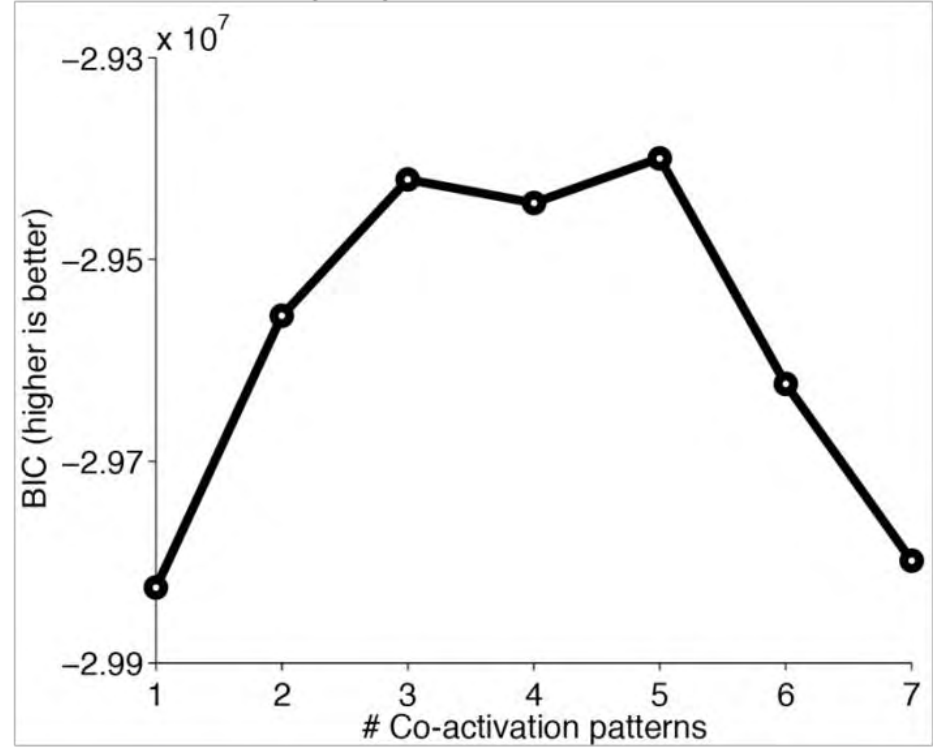

\section{B. Co-activation patterns involving the inferior frontal junction (IFJ)}

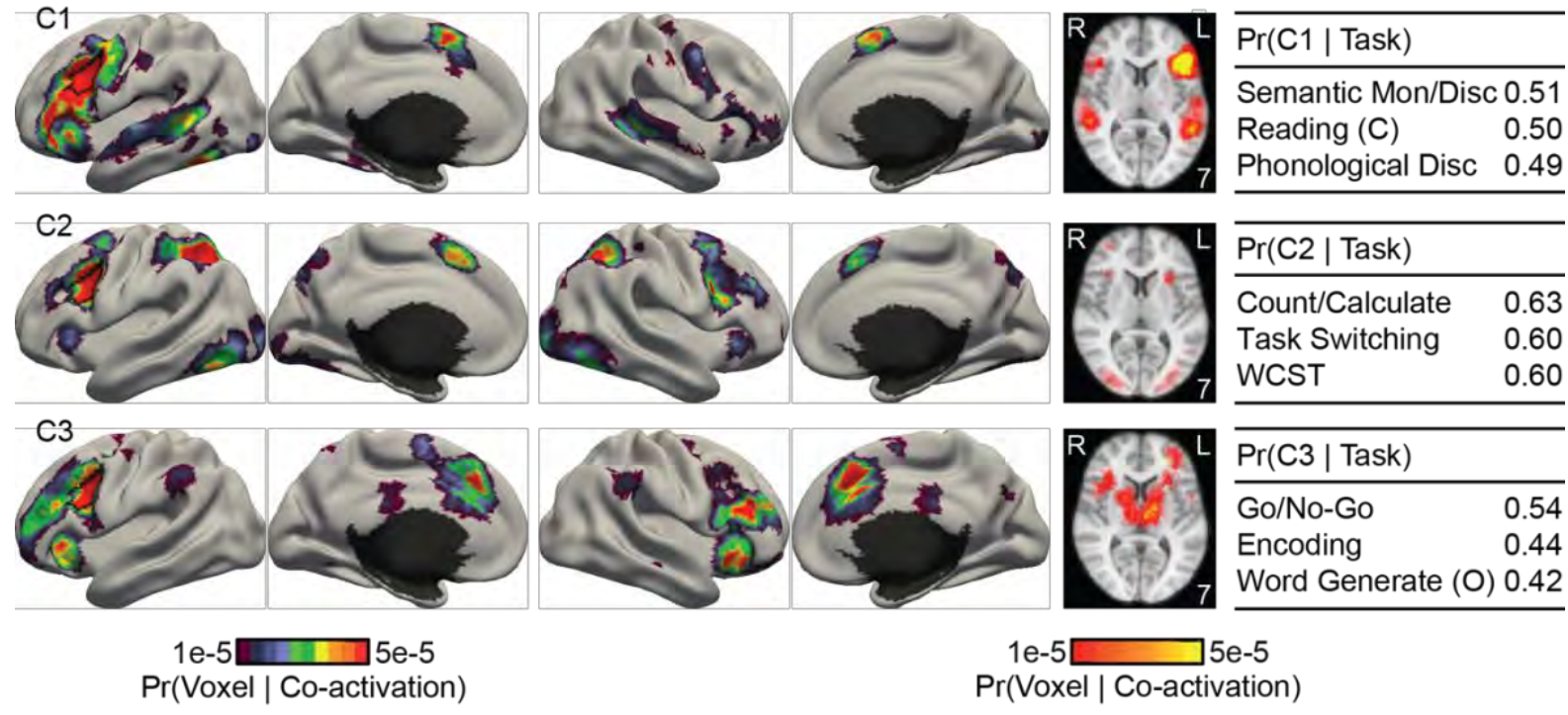

Figure 11. Co-activation patterns involving the inferior frontal junction (IFJ). (A) Bayesian Information Criterion (BIC) plotted as a function of the number of estimated co-activation patterns. BIC peaks at 3 co-activation patterns. (B) 3-coactivation-pattern model estimates for the IFJ. Format follows Figure 7. "(C)" and "(O)" indicate "covert" and "overt" respectively. "Mon", and "Disc" are short for "monitor" and "discrimination" respectively. "Count/ 
Calculate" is short for "Counting/Calculation". "WCST" is short for "Wisconsin Card Sorting Test". The left IFJ is delineated by the black boundary in the left hemisphere.

We now discuss in detail spatial differences among the co-activation patterns. Coactivation pattern $\mathrm{C} 3$ strongly engaged bilateral anterior insula, while co-activation pattern $\mathrm{C} 1$ only engaged left anterior insula. The activation of the anterior insula was much weaker in coactivation pattern $\mathrm{C} 2$.

In the frontal cortex, co-activation pattern $\mathrm{C} 1$ had high probability of activating the left inferior frontal gyrus, while co-activation pattern $\mathrm{C} 3 \mathrm{had}$ high probability of activating bilateral dorsal lateral prefrontal cortex. Although all three co-activation patterns also had high probability of activating the posterior medial frontal cortex (PMFC), the activation shifted anteriorly from co-activation patterns $\mathrm{C} 1$ to $\mathrm{C} 2$ to $\mathrm{C} 3$.

In the parietal cortex, co-activation pattern $\mathrm{C} 2$ included the superior parietal lobule and the intraparietal sulcus in both hemispheres. $\mathrm{C} 1$ and $\mathrm{C} 3$ did not activate the superior parietal cortex. Finally, co-activation pattern $\mathrm{C} 1$ engaged bilateral superior temporal cortex, which might overlap with early auditory regions. Both co-activation patterns $\mathrm{C} 1$ and $\mathrm{C} 2$ also had high probability of activating ventral visual regions, especially in the fusiform gyrus.

The top three tasks recruiting each co-activation pattern is shown in Figure 11B. For completeness, the top five tasks recruiting each co-activation pattern are shown in Table S2. The top tasks with the highest probability of recruiting co-activation pattern $\mathrm{C} 1$ were "Semantic Monitoring/Discrimination", "Covert Reading", and "Phonological Discrimination". The top tasks recruiting co-activation pattern C2 were "Counting/Calculation", "Task Switching", and "Wisconsin Card Sorting Test". The top tasks recruiting co-activation pattern C3 were "Go/ No-Go", "Encoding", and "Overt Word Generation".

At first glance, the top three tasks for co-activation pattern C3 ("Go/ No-Go", "Encoding”, and "Overt Word Generation") might not seem to be similar tasks. The reason for this incongruence was previously explained in Section 2.5.3 and was due to the fact that the experiments activating IFJ might not be representative of their task categories. Indeed, of the 123 BrainMap experiments labeled as the "Overt Word Generation" task, only 6 experiments reported activation in the IFJ. Thus, the 6 experiments were not simply "Overt Word Generation" task, but "Overt Word Generation" experiments that happened to activate the IFJ. This motivated further examination of the original publications associated with the top experiments activating IFJ in order to interpret the co-activation patterns (see Section 4.2 for discussion). 
Table S3A-S3C list the top three experiments with the highest $\operatorname{Pr}$ (co-activation pattern | experiment) for each of the top three tasks associated with each co-activation pattern. For example, Table S3-A lists the top three experiments employing "Semantic Monitoring/Discrimination", "Covert Reading" or "Phonological Discrimination" with the highest $\operatorname{Pr}($ co-activation pattern $\mathrm{C} 1 \mid$ experiment).

\begin{tabular}{lcccc}
\multicolumn{1}{c}{} & $\mathrm{x} / \mathrm{mm}$ & $\mathrm{y} / \mathrm{mm}$ & $\mathrm{z} / \mathrm{mm}$ \\
\hline Co-activation pattern C1 & $-40.33(1.80)$ & $3.89(3.55)$ & $30.67(4.21)$ \\
Co-activation pattern C2 & $-39.33(3.16)$ & $5.33(4.92)$ & $31.78(5.45)$ \\
Co-activation pattern C3 & $-39.40(4.60)$ & $6.40(5.68)$ & $29.70(4.64)$
\end{tabular}

Table 1. Spatial locations of activation foci within the IFJ. Each row of the table shows the mean (standard deviation) of the coordinates of the activation foci (within IFJ) reported by the top 3 experiments with the highest $\operatorname{Pr}$ (co-activation pattern $\mid$ experiment) for each of the top three tasks associated with each co-activation pattern falling inside the IFJ. See Figure S4 for volumetric slices illustrating the locations of the activation foci within the IFJ. Across the 3 co-activation patterns, the mean coordinates of the top experiments do not differ by more than $2.5 \mathrm{~mm}$ in any dimension, suggesting that the co-activation patterns were not fractionating the IFJ.

To further ensure that the 3 co-activation patterns were not fractionating IFJ (like the 5-pattern estimate), Figure S4 illustrates the activation foci of the top three experiments with the highest $\operatorname{Pr}$ (co-activation pattern | experiment) for each of the top three tasks associated with each co-activation pattern falling inside the IFJ. Table 1 shows the mean and standard deviation of the coordinates of these activation foci (within IFJ) for each co-activation pattern. The mean locations of the IFJ activations across co-activation patterns did not differ by more than $2.5 \mathrm{~mm}$ along any dimension, suggesting that the co-activation patterns were probably not simply subdividing the IFJ.

\subsubsection{Goodness offit}

Figure 12 shows the correlation matrix between IFJ's co-activation patterns (columns) and the average activation maps of the top three tasks associated with each co-activation pattern (rows). The diagonal entries of the correlation matrix were significantly higher than the offdiagonal entries: average diagonal entry was 0.75 , while the average off-diagonal entry was 0.31. Overall, this suggests a good model fit. 


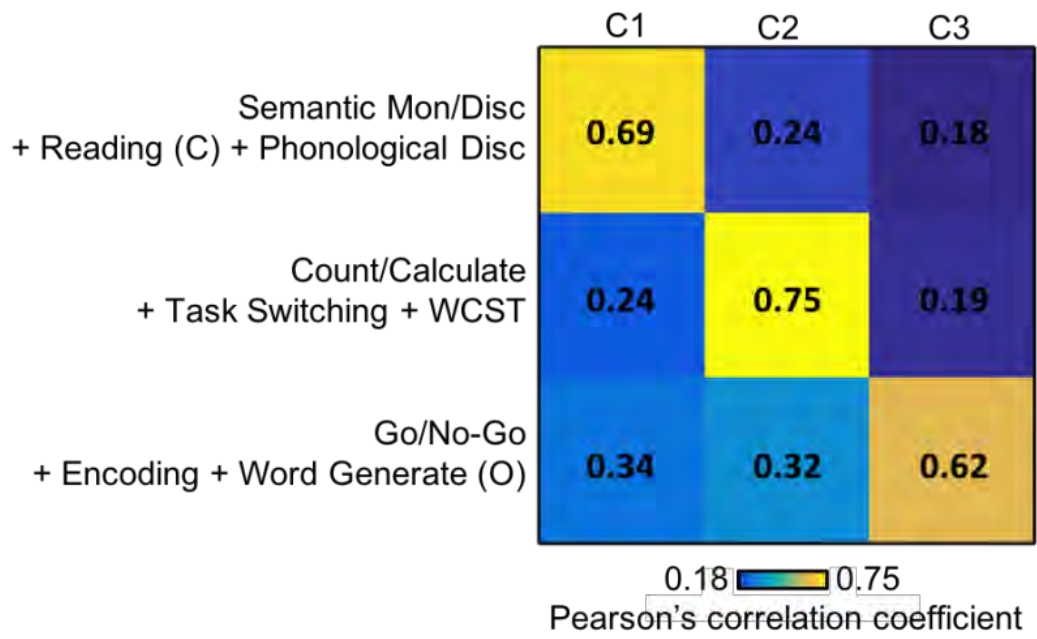

Figure 12. Goodness of fit of the author-topic model for IFJ. The matrix represents the correlations between IFJ's co-activation patterns (columns) and the average activation maps of the top three tasks associated with each co-activation pattern (rows). The top tasks of each coactivation patterns are shown in Figure 11. The diagonal values (average $r=0.75$ ) were larger than off-diagonal values (average $r=0.31$ ), suggesting a good model fit.

\subsection{Control analyses}

\subsubsection{Smoothing}

To create the input data for the author-topic model, the activation foci were smoothed with a $10 \mathrm{~mm}$ binary smoothing kernel (see Section 2.2.3), consistent with previous work (Wager et al. 2003; Yarkoni et al. 2011; Yeo et al. 2015). Using different smoothing radii yielded similar cognitive components of self-generated thought (Figure S5) and co-activation patterns of the IFJ (Figure S6).

\subsubsection{Independent component analysis}

For comparison, Figure S7 shows the ICA (ICA1-nilearn) estimate of 2 components of self-generated thought. The estimates were quite similar to the author-topic estimate. However, the spatial maps contained negative values, which was inappropriate in the context of coordinate-based meta-analysis. 
Figure S8 shows the ICA (ICA1-nilearn) estimate of 3 co-activation patterns of left IFJ. However, the 3 independent components appeared to fractionate the left IFJ into smaller territories (Figure S8), suggesting a degenerate solution to our problem, similar to the situation with the 5-pattern author-topic estimate (Figure S3). Furthermore, both the mixture weights and spatial maps contained negative values, which were not interpretable in the context of coordinate-based meta-analysis (Section 2.3.1). 


\section{Discussion}

The author-topic model encodes the intuitive notion that behavioral tasks recruited multiple cognitive components, supported by multiple brain regions (Mesulam 1990; Poldrack 2006; Barrett \& Satpute, 2013). We have previously utilized the author-topic model for largescale meta-analysis across functional domains (Yeo et al., 2015; Bertolero et al., 2015). By exploiting a recently developed CVB algorithm for the author-topic model (Ngo et al., 2016), we show that the model can also be utilized for small-scale meta-analyses focusing on discovering functional sub-domains or task-dependent co-activation patterns.

A dominant approach for small-scale meta-analyses is ALE, which seeks to find consistent activations across neuroimaging experiments within a functional domain or mental disorder or seed region (also known as MACM). ALE treats heterogeneity across experiments as noise. By contrast, the author-topic model evaluates whether the heterogeneity might be indicative of robust latent patterns within the data. We applied the author-topic model to two applications: one on fractionating a functional sub-domain and one on discovering multiple task-dependent co-activation patterns.

In the first application, the author-topic model encoded the notion that tasks involving self-generated thought might recruit one or more spatially overlapping. cognitive components. The model revealed two cognitive components that appeared to delineate two overlapping default sub-networks, consistent with the hypothesized functional organization of the default network (Andres-Hanna et al., 2014). In the second application, the author-topic model encoded the notion that experiments activating a brain region might recruit one or more coactivation patterns dependent on task contexts (McIntosh, 2000). In the current application, the model revealed that the IFJ participated in three co-activation patterns, suggesting that IFJ flexibly co-activate with different brain regions depending on the cognitive demands of different tasks. Overall, our work suggests that the author-topic model is a versatile tool suitable for both small-scale and large-scale meta-analyses.

\subsection{Cognitive components of self-generated thought}

Self-generated thought is a heterogeneous set of cognitive processes that includes inferring other people's mental states, dealing with challenging moral scenarios, understanding narratives, retrieving autobiographical memories, internalizing semantic information, and mind-wandering. These processes are characterized by an absence of external stimuli, selfrelated, and often involve simulation or inferential reasoning (Buckner et al., 2008; Spreng et al. 2009; Smallwood et al., 2011; Baird et al., 2011; Prebble at al. 2013; Smallwood, 2013). 
Studies of tasks involving self-generated thought have consistently found the activation of the default network, suggesting its functional importance (Buckner et al. 2008; Spreng et al. 2009; Andrews-Hanna et al., 2010; Andrews-Hanna, 2012; Gorgolewski et al., 2014; Callard and Margulies, 2014). Additionally, the default network has been fractionated into sub-networks supporting different aspects of these stimulus independent cognitive processes (Buckner et al., 2008; Uddin et al. 2009; Sestieri et al., 2011; Andrews-Hanna et al., 2010; Kim, 2012; Seghier and Price, 2012; Salomon et al., 2013; Bzdok et al., 2013).

The author-topic model revealed two cognitive components of self-generated thought that appeared to fractionate the default network (Figure 7). The default network has been defined as the set of brain regions that are more active during passive task conditions relative to active task conditions (Shulman et al., 1997; Buckner et al., 2008). While there have been multiple studies fractionating the default network (Andrews-Hanna et al., 2010; Mayer et al. 2010; Kim, 2012; Yeo et al. 2014; Humphreys et al., 2015), the specific patterns of fractionation have differed across studies. The spatial topography of components $\mathrm{C} 1$ and $\mathrm{C} 2$ in this paper corresponded well to the previously proposed "medial temporal subsystem" and "dorsal medial subsystem" respectively (Figure 3A of Andrews-Hanna et al. 2014; AndrewsHanna et al., 2010).

The first cognitive component $\mathrm{C} 1$ was strongly recruited by navigation and autobiographical memory tasks, suggesting its involvement in constructive mental simulation based upon mnemonic content (Andrews-Hanna et al., 2014). Constructive mental simulation is the process of combining information from the past in order to create a novel mental representation, such as imagining the future (Buckner and Carroll, 2007; Hassabis and Maguire, 2007; Schacter et al., 2007). "Navigation" tasks require constructive mental simulation to create a mental visualization ("scene construction") for planning new routes and finding ways in unfamiliar contexts (Burgess et al., 2002; Byrne et al. 2007). On the other hand, "Autobiographical Memory" tasks require constructive mental simulation to project past experience ("constructive episodic simulation"; Atance and O’Neil, 2001; Schacter et al. 2007) or previously acquired knowledge ("semantic memory"; Irish et al., 2012; Brown et al. 2014) across spatiotemporal scale to enact novel perspectives. Overall, cognitive component $\mathrm{C} 1$ seems to support the projection of self, events, experiences, images and knowledge to a new temporal or spatial context based upon an associative constructive process, likely mediated by the hippocampus and connected brain structures (Moscovitch et al., 2016, Christoff et al., 2016). 
The second cognitive component $\mathrm{C} 2$ was strongly recruited by narrative comprehension and theory of mind, suggesting its involvement in mentalizing, inferential, and conceptual processing (Andrews-Hanna et al., 2014). Mentalizing is the process of monitoring one's own mental states or predicting others' mental states (Frith and Frith, 2003), while conceptual processing involves internalizing and retrieving semantic or social knowledge (Binder and Desai, 2011; Overwalle, 2009). "Narrative Comprehension" engages conceptual processing to understand the contextual settings of the story, and requires mentalizing to follow and infer the characters' thoughts and emotions (Gernsbacher et al., 1998; Mason et al. 2008). "Theory of Mind" tasks require the recall of learned knowledge, social norms and attitudes to form a meta-representation of the perspectives of other people (Leslie, 1987; Frith and Frith, 2005; Binder and Desai, 2011). The grouping of Narrative Comprehension and Theory of Mind tasks echoes the link between the ability to comprehend narratives and the ability to understanding others' thoughts in developmental studies of children (Guajardo and Watson, 2001; Slaughter et al. 2007; Mason et al. 2008).

The two cognitive components had high probability of activating common and distinct brain regions. Both components engaged the posterior cingulate cortex and precuneus, which are considered part of the "core" sub-network that subserves personally relevant information necessary for both constructive mental simulation and mentalizing (Andrews-Hanna et al. 2014). The distinct brain regions supporting each cognitive component also corroborated the distinct functional role of each component. For instance, component $\mathrm{C} 1$, but not $\mathrm{C} 2$, had high probability of activating the medial temporal lobe and hippocampus. This is consistent with neuropsychological literaure showing that patients with impairment of the medial temporal lobe and hippocampus retain theory of mind and narrative construction capabilities, while suffering deficits in episodic memories and imagining the future (Hassabis et al., 2007; Rosenbaum et al., 2007; Rosenbaum et al., 2009; Race et al., 2011;).

The cognitive components of self-generated thought estimated by the author-topic model overlapped with default sub-networks A, B and C, as well as the temporal parietal network from a previously published resting-state parcellation (Yeo et al., 2011; Kong et al., 2018). The components loaded differentially on the resting-state networks, thus providing insights into the functions of distinct resting-state networks. Although resting-state fMRI is a powerful tool for extracting brain networks, participants do not actively perform a task during resting-state fMRI. Thus, coordinate-based meta-analysis can be used in conjunction with resting-state fMRI to discover new insights into brain networks and their functions (Seeley et al., 2007; Smith et al., 2009; Laird et al., 2011). 


\subsection{Co-activation patterns of the left IFJ}

The inferior frontal junction (IFJ) is located in the prefrontal cortex at the intersection between the inferior frontal sulcus and the inferior precentral sulcus (Brass et al., 2005; Derrfuss et al., 2005). The IFJ has been suggested to be involved in a wide range of cognitive functions, including task switching (Brass and Cramon, 2002; Derrfuss et al., 2004, 2005), attentional control (Asplund et al. 2010; Baldauf and Desimone, 2014), detection of conflicting responses (Chikazoe et al. 2009; Levy and Wagner, 2011), short-term memory (Zanto et al. 2010; Sneve et al. 2013), construction of attentional episodes (Duncan, 2013) and so on. Using the author-topic model, we found that the IFJ participated in three task-dependent co-activation patterns.

Co-activation pattern Cl might be involved in some aspects of language processing, such as phonological processing for lexical understanding. Phonological processing is an important linguistic function, concerning the use of speech sounds in handling written or oral languages (Wagner and Torgesen 1987; Poldrack et al. 1999; Friederici 2002). The top tasks associated with C1 were "Semantic Monitoring/ Discrimination", "Covert Reading", and "Phonological Discrimination" (Figure 11B). Inspecting the top three experiments recruiting these three tasks (Table S3-A) offered more insights into the functional characteristics of coactivation pattern C1. The top "Semantic Monitor/Discrimination" experiments with the highest probability of recruiting co-activation pattern $\mathrm{C} 1$ examined retrieval of semantic meaning (Thompson-Schill et al. 1999; Wagner et al. 2001) and an experiment requiring lexical perception and not just perception of elementary sounds (Poeppel et al., 2004). The top "Covert Reading” experiments most strongly associated with $\mathrm{C} 1$ identified a common brain network activated by both reading and listening (Jobard et al., 2007), as well as language comprehension across different media (Small et al., 2009), suggesting the involvement of $\mathrm{C} 1$ in generic language comprehension. Among "Phonological Discrimination" experiments, C1 was most highly associated with experiments engaging transcoding of phonological representation for semantic perception (Xu et al. 2001; Démonet et al. 1994). The language and phonological processing interpretation was supported by C1's strong left lateralization with high probability of activating classical auditory and language brain regions, including the left (but not right) inferior frontal gyrus and bilateral superior temporal cortex.

Co-activation pattern C2 might be engaged in attentional control, especially aspects of task maintenance and shifting of attentional set. Attentional set-shifting is the ability to switch between mental states associated with different reactionary tendencies (Omori et al. 1999, Konishi et al. 1998). The top three tasks most highly associated with C2 were 
“Counting/Calculation”, "Task Switching”, and "Wisconsin Card Sorting Test” (Figure 11B). Inspecting the top three experiments under the top task paradigms provided further insights into the functional characteristics of co-activation pattern C2 (Table S3-B). The top "Counting/ Calculation" experiments most strongly recruiting co-activation pattern $\mathrm{C} 2$ involved switching of resolution strategies in executive function. For example, one experimental contrast seeks to isolate demanding mental calculation but not retrieval of numerical facts (Zago et al. 2001; Rivera et al. 2002), suggesting C2's involvement in the selection and application of strategies to solve arithmetic problems. The top "Task Switching" experiments most strongly associated with C2 involved the switching of mental states to learn new stimulus-response or stimulusoutcome associations (Omori et al. 1999; Nahagama et al. 2001; Sylvester et al. 2003). C2 was also strongly expressed by "Wisconsin Card Sorting Test" (WCST) experiments, which required attentional set-shifting to change behavioral patterns in reaction to changes of perceptual dimension (color, shape, or number) upon which the target and reference stimuli were matched (Berman 1995; Konishi 2002; Konishi 2003). Overall, the attentional control interpretation of co-activation pattern C2 is supported by C2's high probability of activating classical attentional control regions, such as the superior parietal lobule and the intra-parietal sulcus, although there is a clear lack of DLPFC activation.

Co-activation pattern C3 might be engaged in inhibition or response conflict resolution. Conflict-response resolution is a central aspect of cognitive control, which involves monitoring and mediating incongruous response tendencies (Pardo et al. 1990; Braver et al. 2001; Barch et al. 2001). Co-activation pattern $\mathrm{C} 3$ is most strongly recruited by experiments utilizing "Go/No-Go", "Encoding" and "Overt Word Generation" tasks (Figure 11B). Closer examination of the top three experiments under each task paradigm provided further insights into the functional characteristics of C3 (Table S3-C). The top experiments utilizing "Go/NoGo" required the monitoring, preparing and reconciling of conflicting tendencies to either giving a "go" or "stop" (no-go) response (Chikazoe et al. 2009, Simoes-Franklin et al. 2010; Kawashima et al. 1996). It might be surprising at first glance that the "Go/No-Go" task was grouped together with "Encoding" and "Overt Word Generation" tasks. However, the top experiments utilizing the "Encoding" and "Overt Word Generation" task all required subjects to make competing decisions (Table S3-C). The top "Encoding" experiments most strongly associated with $\mathrm{C} 3$ required selective association of to-be-learnt items with existing memory or knowledge organization for effective enduring retention of new information (Kapur et al. 1996; Callan et al. 2010; Mickley et al. 2009). The top experiments utilizing the "Overt Word Generation" task required subjects to make competing decision, such as inhibiting 
verbalization of wrong words in verbal fluency task (Baker et al. 1997; Ravnkilde et al. 2002) or inhibiting a predominant pattern (regular past-tense verbs) in favor of generating less conventional forms (irregular past-tense verbs) (Desai et al. 2006). Overall, the inhibition or response conflict interpretation of co-activation pattern C3 is supported by C3's high probability of activating classical executive function regions, including the bilateral dorsal lateral prefrontal cortex.

The intriguing location of the left IFJ and its functional heterogeneity suggests the role of IFJ as an integrative hub for different cognitive functions. For example, the IFJ has been suggested to consolidate information streams for cognitive control from its bordering brain regions (Brass et al., 2005). The involvement of the IFJ in three task-dependent co-activation patterns supported the view that the IFJ orchestrates different cognitive mechanisms to allow their operations in harmony.

\section{Conclusion}

Heterogeneities across neuroimaging experiments are often treated as noise in coordinate-based meta-analyses. Here we demonstrate that the author-topic model can be utilized to determine if the heterogeneities can be explained by a small number of latent patterns. In the first application, the author-topic model revealed two overlapping cognitive components subserving self-generated thought. In the second application, the author-topic revealed the participation of the left IFJ in three task-dependent co-activation patterns. These applications exhibited the broad utility of the author-topic model, ranging from discovering functional subdomains or task-dependent co-activation patterns.

\section{Acknowledgements}

This work was supported by Singapore MOE Tier 2 (MOE2014-T2-2-016), NUS Strategic Research (DPRT/944/09/14), NUS SOM Aspiration Fund (R185000271720), Singapore NMRC (CBRG/0088/2015), NUS YIA and the Singapore National Research Foundation (NRF) Fellowship (Class of 2017). Simon Eickhoff is supported by the National Institute of Mental Health (R01-MH074457), the Helmholtz Portfolio Theme "Supercomputing and Modeling for the Human Brain" and the European Union's Horizon 2020 Research and Innovation Programme under Grant Agreement No. 7202070 (HBP SGA1). R. Nathan Spreng is supported by the National Science and Engineering Research Council of Canada, the Canadian Institutes of Health Research, and received salary support from the Fonds de la Recherche du Quebec - Santé (FRQS). Comprehensive access to the BrainMap database was 
authorized by a collaborative-use license agreement (http://www.brainmap.org/collaborations.html). BrainMap database development is supported by NIH/NIMH R01 MH074457. Our computational work was partially performed on resources of the National Supercomputing Centre, Singapore (https://www.nscc.sg). 


\section{References}

Abraham A, Pedregosa F, Eickenberg M, Gervais P, Mueller A, Kossaifi J, Gramfort A, Thirion B, Varoquaux G. 2014. Machine learning for neuroimaging with scikit-learn. Frontiers in Neuroinformatics. 8:14.

Andrews-Hanna JR, Reidler JS, Huang C, Buckner RL. 2010. Evidence for the default networks role in spontaneous cognition. Journal of Neurophysiology. 104:322-335.

Andrews-Hanna JR, Reidler JS, Sepulcre J, Poulin R, Buckner RL. 2010. Functionalanatomic fractionation of the brains default network. Neuron. 65:550-562.

Andrews-Hanna JR, Smallwood J, Spreng, RN. 2014. The default network and self-generated thought: component processes, dynamic control, and clinical relevance. Annals of the New York Academy of Sciences. 1316:29-52.

Andrews-Hanna JR. 2012. The brain's default network and its adaptive role in internal mentation. The Neuroscientist. 18:251-270.

Asplund CL, Todd JJ, Snyder AP, Marois R. 2010. A central role for the lateral prefrontal cortex in goal-directed and stimulus-driven attention. Nature Neuroscience. 13:507-512.

Atance CM, O’Neill DK. 2001. Episodic future thinking. Trends in Cognitive Sciences. 5:533-539.

Baird B, Smallwood J, Schooler JW. 2011. Back to the future: autobiographical planning and the functionality of mind-wandering. Consciousness and Cognition. 20:1604-1611.

Baker SC, Frith CD, Dolan RJ. 1997. The interaction between mood and cognitive function studied with PET. Psychological Medicine. 27:565-78.

Baldauf D, Desimone R. 2014. Neural mechanisms of object-based attention. Science. 344:424-427.

Barch DM, Braver TS, Akbudak E, Conturo T, Ollinger J, Snyder A. 2001. Anterior cingulate cortex and response conflict: effects of response modality and processing domain. Cerebral Cortex. 11:837-848.

Barrett LF, Satpute AB. 2013. Large-scale brain networks in affective and social neuroscience: Towards an integrative functional architecture of the brain. Current Opinion in Neurobiology. 23:361-372.

Beal MJ. 2003. Variational algorithms for approximate Bayesian inference, University of London United Kingdom.

Beckmann CF, Smith SM. 2004. Probabilistic independent component analysis for functional magnetic resonance imaging. IEEE Transactions on Medical Imaging. 23:137-52.

Beissner F, Meissner K, Bär KJ, Napadow V. 2013. The autonomic brain: an activation likelihood estimation meta-analysis for central processing of autonomic function. Journal of Neuroscience. 33:10503-10511.

Berman KF, Ostrem JL, Randolph C, Gold J, Goldberg TE, Coppola R, Carson RE, Herscovitch P, Weinberger DR. 1995. Physiological activation of a cortical network during performance of the Wisconsin Card Sorting Test: a positron emission tomography study. Neuropsychologia. 33:1027-1046.

Bertolero MA, Yeo BTT, D'Esposito M. 2015. The modular and integrative functional architecture of the human brain. Proceedings of the National Academy of Sciences. 112:E6798-E6807.

Bertolero MA, Yeo BT, D’Esposito M. 2017. The diverse club. Nature communications. 8:1277.

Bertolero MA, Yeo BTT, Bassett SD, D'Esposito M. 2018. A mechanistic model of connector hubs, modularity, and cognition. Nature Human Behaviour. 112: E6798.

Binder JR, Desai RH, Graves WW, Conant LL. 2009. Where is the semantic system? A critical review and meta-analysis of 120 functional neuroimaging studies. Cerebral 
Cortex. 19:2767-2796.

Binder JR, Desai RH. 2011. The neurobiology of semantic memory. Trends in Cognitive Sciences. 15:527-536.

Braga RM, Buckner RL. 2017. Parallel interdigitated distributed networks within the individual estimated by intrinsic functional connectivity. Neuron. 9:457-71.

Brass M, Derrfuss J, Forstmann B, von Cramon DY. 2005. The role of the inferior frontal junction area in cognitive control. Trends in Cognitive Sciences. 9:314-316.

Brass M, von Cramon DY. 2002. The role of the frontal cortex in task preparation. Cerebral Cortex. 12:908-914.

Braver TS, Barch DM, Gray JR, Molfese DL, Snyder A. 2001. Anterior cingulate cortex and response conflict: effects of frequency, inhibition and errors. Cerebral Cortex. 11:825836.

Brown AD, Addis DR, Romano TA, Marmar CR, Bryant RA, Hirst W, Schacter DL. 2014. Episodic and semantic components of autobiographical memories and imagined future events in post-traumatic stress disorder. Memory. 22:595-604.

Buckner RL, Andrews-Hanna JR, Schacter DL. 2008. The brains default network. Annals of the New York Academy of Sciences. 1124:1-38.

Buckner RL, Carroll DC. 2007. Self-projection and the brain. Trends in Cognitive Sciences. 11:49-57.

Buckner RL, Krienen FM, Castellanos A, Diaz JC, Yeo BTT. 2011. The organization of the human cerebellum estimated by intrinsic functional connectivity. Journal of Neurophysiology. 106:2322-2345.

Burgess N, Maguire EA, OKeefe J. 2002. The human hippocampus and spatial and episodic memory. Neuron. 35:625-641.

Button KS, Ioannidis JP, Mokrysz C, Nosek BA, Flint J, Robinson ES, Munafò MR. 2013. Power failure: why small sample size undermines the reliability of neuroscience. Nature Reviews. Neuroscience. 14:365.

Byrne P, Becker S, Burgess N. 2007. Remembering the past and imagining the future: a neural model of spatial memory and imagery. Psychological Review. 114:340.

Bzdok D, Langner R, Schilbach L, Engemann DA, Laird AR, Fox PT, Eickhoff S. 2013. Segregation of the human medial prefrontal cortex in social cognition. Frontiers in Human Neuroscience. 7:232.

Calhoun VD, Adali T, Pearlson GD, Pekar JJ. 2001. A method for making group inferences from functional MRI data using independent component analysis. Human Brain Mmapping. 14:140-51.

Callan DE, Schweighofer N. 2010. Neural correlates of the spacing effect in explicit verbal semantic encoding support the deficient - processing theory. Human Brain Mapping. 31:645-59.

Callard F, Margulies DS. 2014. What we talk about when we talk about the default mode network. Frontiers in Human Neuroscience. 8:619.

Carp J. 2012. The secret lives of experiments: methods reporting in the fMRI literature. Neuroimage. 63:289-300.

Chein JM, Fissell K, Jacobs S, Fiez JA. 2002. Functional heterogeneity within Brocas area during verbal working memory. Physiology, Behavior. 77:635-639.

Chikazoe J, Jimura K, Asari T, Yamashita K, Morimoto H, Hirose S, Miyashita Y, Konishi S. 2009. Functional dissociation in right inferior frontal cortex during performance of go/no-go task. Cerebral Cortex. 19:146-152.

Chikazoe J, Jimura K, Hirose S, Yamashita K, Miyashita Y, Konishi S. 2009. Preparation to inhibit a response complements response inhibition during performance of a stop-signal task. Journal of Neuroscience. 29:15870-15877. 
Christoff K, Irving ZC, Fox KCR, Spreng RN, Andrews-Hanna JR. 2016. Mind-wandering as spontaneous thought: A dynamic framework. Nature Reviews Neuroscience. 17:718731.

Cole MW, Reynolds JR, Power JD, Repovs G, Anticevic A, Braver TS. 2013. Multi-task connectivity reveals flexible hubs for adaptive task control. Nature Neuroscience. $16: 1348$.

Cortese S, Kelly C, Chabernaud C, Proal E, Di Martino A, Milham MP, Castellanos FX. 2012. Toward systems neuroscience of ADHD: a meta-analysis of $55 \mathrm{fMRI}$ studies. American Journal of Psychiatry. 169:1038-1055.

Costafreda SG, Brammer MJ, David AS, Fu CHY. 2008. Predictors of amygdala activation during the processing of emotional stimuli: a meta-analysis of 385 PET and fMRI studies. Brain Research Reviews. 58:57-70.

Cox RW. 1996. AFNI: software for analysis and visualization of functional magnetic resonance neuroimages. Computers and Biomedical research. 29:162-173.

Crossley NA, Mechelli A, Scott J, Carletti F, Fox PT, McGuire P, Bullmore ET. 2014. The hubs of the human connectome are generally implicated in the anatomy of brain disorders. Brain. 137:2382-2395.

Delaveau P, Jabourian M, Lemogne C, Guionnet S, Bergouignan L, Fossati P. 2011. Brain effects of antidepressants in major depression: a meta-analysis of emotional processing studies. Journal of Affective Disorders. 130:66-74.

Démonet JF, Price C, Wise R, Frackowiak RS. 1994. A PET study of cognitive strategies in normal subjects during language tasks: Influence of phonetic ambiguity and sequence processing on phoneme monitoring. Brain. 117:671-82.

Derrfuss J, Brass M, Neumann J, von Cramon DY. 2005. Involvement of the inferior frontal junction in cognitive control: Meta-analyses of switching and Stroop studies. Human Brain Mapping. 25:22-34.

Derrfuss J, Brass M, Von Cramon DY. 2004. Cognitive control in the posterior frontolateral cortex: evidence from common activations in task coordination, interference control, and working memory. Neuroimage. 23:604-612.

Desai R, Conant LL, Waldron E, Binder JR. 2006. FMRI of past tense processing: the effects of phonological complexity and task difficulty. Journal of Cognitive Neuroscience. 18:278-97.

Di Martino A, Ross K, Uddin LQ, Sklar AB, Castellanos FX, Milham MP. 2009. Functional brain correlates of social and nonsocial processes in autism spectrum disorders: an activation likelihood estimation meta-analysis. Biological Psychiatry. 65:63-74.

Duncan J. 2010. The multiple-demand (MD) system of the primate brain: mental programs for intelligent behaviour. Trends in cognitive sciences. 14:172-179.

Duncan J. 2013. The structure of cognition: attentional episodes in mind and brain. Neuron. 80:35-50.

Eickhoff SB, Bzdok D, Laird AR, Kurth F, Fox PT. 2012. Activation likelihood estimation meta-analysis revisited. Neuroimage. 59:2349-2361.

Eickhoff SB, Jbabdi S, Caspers S, Laird AR, Fox PT, Zilles K, Behrens TEJ. 2010. Anatomical and functional connectivity of cytoarchitectonic areas within the human parietal operculum. Journal of Neuroscience. 30:6409-6421.

Eickhoff SB, Laird AR, Grefkes C, Wang LE, Zilles K, Fox PT. 2009. Coordinate-based activation likelihood estimation meta-analysis of neuroimaging data: A random-effects approach based on empirical estimates of spatial uncertainty. Human Brain Mapping. 30:2907-2926.

Eickhoff SB, Yeo BT, Genon S. 2018. Imaging-based parcellations of the human brain. Nature Reviews Neuroscience. 1. 
Fedorenko E, Duncan J, Kanwisher N. 2013. Broad domain generality in focal regions of frontal and parietal cortex. Proceedings of the National Academy of Sciences.

110:16616-16621.

Fischl B. 2012. FreeSurfer. Neuroimage. 62:774-781.

Fitzgerald PB, Laird AR, Maller J, Daskalakis ZJ. 2008. A meta-analytic study of changes in brain activation in depression. Human Brain Mapping. 29:683-695.

Fox PT, Lancaster JL, Laird AR, Eickhoff SB. 2014. Meta-analysis in human neuroimaging: computational modeling of large-scale databases. Annual Review of Neuroscience. 37:409-434.

Fox PT, Lancaster JL. 2002. Mapping context and content: the BrainMap model. Nature Reviews Neuroscience. 3:319-321.

Friederici AD. 2002. Towards a neural basis of auditory sentence processing. Trends in Cognitive Sciences. 6:78-84.

Frith C, Frith U. 2005. Theory of mind. Current Biology. 15:R644-R645.

Frith U, Frith CD. 2003. Development and neurophysiology of mentalizing. Philosophical Transactions of the Royal Society B: Biological Sciences. 358:459-473.

Gernsbacher MA, Hallada BM, Robertson RRW. 1998. How automatically do readers infer fictional characters emotional states?. Scientific Studies of Reading. 2:271-300.

Gorgolewski KJ, Lurie D, Urchs S, Kipping JA, Craddock RC, Milham MP, Margulies DS, Smallwood J. 2014. A correspondence between individual differences in the brain's intrinsic functional architecture and the content and form of self-generated thoughts. PloS one. 9:e97176.

Gorgolewski KJ, Varoquaux G, Rivera G, Schwartz Y, Ghosh SS, Maumet C, Sochat VV, Nichols TE, Poldrack RA, Poline J-B, Yarkoni T and Margulies DS. 2015.

NeuroVault.org: a web-based repository for collecting and sharing unthresholded statistical maps of the brain. Frontiers in Neuroinformatics. 9:8.

Guajardo NR, Watson AC. 2002. Narrative discourse and theory of mind development. The Journal of Genetic Psychology. 163:305-325.

Hassabis D, Kumaran D, Vann SD, Maguire EA. 2007. Patients with hippocampal amnesia cannot imagine new experiences. Proceedings of the National Academy of Sciences. 104:1726-1731.

Hassabis D, Maguire EA. 2007. Deconstructing episodic memory with construction. Trends in Cognitive Sciences. 11:299-306.

Humphreys GF, Hoffman P, Visser M, Binney RJ, Ralph MAL. 2015. Establishing task-and modality-dependent dissociations between the semantic and default mode networks. Proceedings of the National Academy of Sciences. 112:7857-7862.

Irish M, Addis DR, Hodges JR, Piguet O. 2012. Considering the role of semantic memory in episodic future thinking: evidence from semantic dementia. Brain. 135:2178-2191.

Jobard G, Vigneau M, Mazoyer B, Tzourio-Mazoyer N. 2007. Impact of modality and linguistic complexity during reading and listening tasks. Neuroimage. 34:784-800.

Kapur S, Tulving E, Cabeza R, McIntosh AR, Houle S, Craik FI. 1996. The neural correlates of intentional learning of verbal materials: a PET study in humans. Cognitive Brain Research. 4:243-9.

Kawashima R, Satoh K, Itoh H, Ono S, Furumoto S, Gotoh R, Koyama M, Yoshioka S, Takahashi T, Takahashi K, Yanagisawa T. 1996. Functional anatomy of GO/NO-GO discrimination and response selection — a PET study in man. Brain research. 728:79-89

Kernbach JM, Yeo BTT, Smallwood J, Margulies DS, Thiebaut de Schotten M. Walter H, Sabuncu MR, Holmes AJ, Gramfort A, Varoquaux G, Thirion B, Bzdok D. 2018. Subspecialization within default mode nodes characterized in 10,000 UK Biobank participants. Proceedings of the National Academy of Sciences. 201804876 
Kim C, Johnson NF, Cilles SE, Gold BT. 2011. Common and distinct mechanisms of cognitive flexibility in prefrontal cortex. Journal of Neuroscience. 31:4771-4779.

Kim H. 2012. A dual-subsystem model of the brains default network: self-referential processing, memory retrieval processes, and autobiographical memory retrieval. Neuroimage. 61:966-977.

Kiviniemi V, Kantola JH, Jauhiainen J, Hyvärinen A, Tervonen O. 2003. Independent component analysis of nondeterministic fMRI signal sources. Neuroimage. 19:253-60.

Knutson B, Wimmer GE, Rick S, Hollon NG, Prelec D, Loewenstein G. 2008. Neural antecedents of the endowment effect. Neuron. 58:814-822.

Kong R, Li J, Orban C, Sabuncu MR, Liu H, Schaefer A, Sun N, Zuo XN, Holmes A, Eickhoff SB, Yeo BTT. (in press). Spatial topography of individual-specific cortical networks predicts human cognition, personality and emotion. Cerebral Cortex.

Konishi S, Hayashi T, Uchida I, Kikyo H, Takahashi E, Miyashita Y. 2002. Hemispheric asymmetry in human lateral prefrontal cortex during cognitive set shifting. Proceedings of the National Academy of Sciences. 99:7803-7808.

Konishi S, Jimura K, Asari T, Miyashita Y. 2003. Transient activation of superior prefrontal cortex during inhibition of cognitive set. Journal of Neuroscience. 23:7776-7782.

Konishi S, Nakajima K, Uchida I, Kameyama M, Nakahara K, Sekihara K, Miyashita Y. 1998. Transient activation of inferior prefrontal cortex during cognitive set shifting. Nature Neuroscience. 1:80-84.

Koski L, Paus T. 2000. Functional connectivity of the anterior cingulate cortex within the human frontal lobe: a brain-mapping meta-analysis. Executive Control and the Frontal Lobe: Current Issues. 55-65.

Kühn S, Gallinat J. 2013. Resting-state brain activity in schizophrenia and major depression: a quantitative meta-analysis. Schizophrenia Bulletin. 39:358-365.

Laird AR, Eickhoff SB, Li K, Robin DA, Glahn DC, Fox PT. 2009. Investigating the functional heterogeneity of the default mode network using coordinate-based metaanalytic modeling. Journal of Neuroscience. 29:14496-14505.

Laird AR, Eickhoff SB, Kurth F, Fox PM, Uecker AM, Turner JA, Robinson JL, Lancaster JL, Fox PT. 2009. ALE meta-analysis workflows via the Brainmap database: progress towards a probabilistic functional brain atlas. Frontiers in neuroinformatics. 3:23.

Laird AR, Fox PM, Eickhoff SB, Turner JA, Ray KL, McKay DR, Glahn DC, Beckmann CF, Smith SM, Fox PT. 2011. Behavioral interpretations of intrinsic connectivity networks. Journal of Cognitive Neuroscience. 23:4022-4037.

Laird AR, Fox PM, Price CJ, Glahn DC, Uecker AM, Lancaster JL, Turkeltaub PE, Kochunov P, Fox PT. 2005. ALE meta-analysis: Controlling the false discovery rate and performing statistical contrasts. Human brain mapping. 25:155-164.

Lancaster JL, Tordesillas-Gutiérrez D, Martinez M, Salinas F, Evans A, Zilles K, Mazziotta JC, Fox PT. 2007. Bias between MNI and Talairach coordinates analyzed using the ICBM-152 brain template. Human Brain Mapping. 28:1194-1205.

Leech R, Braga R, Sharp DJ. 2012. Echoes of the brain within the posterior cingulate cortex. Journal of Neuroscience. 32:215-222.

Leslie AM. 1987. Pretense and representation: The origins of "theory of mind". Psychological Review. 94:412.

Levy BJ, Wagner AD. 2011. Cognitive control and right ventrolateral prefrontal cortex: reflexive reorienting, motor inhibition, and action updating. Annals of the New York Academy of Sciences. 1224:40-62.

Li J, Kong R, Liegeois R, Orban C, Sun N, Holmes AJ, Sabuncu MR, Ge T, Yeo BTT. Under Review. Global Signal Regression Strengthens Association between Resting-State Functional Connectivity and Behavior. 
Mar RA. 2011. The neural bases of social cognition and story comprehension. Annual Review of Psychology. 62:103-134.

Mason RA, Williams DL, Kana RK, Minshew N, Just MA. 2008. Theory of mind disruption and recruitment of the right hemisphere during narrative comprehension in autism. Neuropsychologia. 46:269-280.

Mason RA, Williams DL, Kana RK, Minshew N, Just MA. 2008. Theory of mind disruption and recruitment of the right hemisphere during narrative comprehension in autism. Neuropsychologia. 46:269-280.

Mayer JS, Roebroeck A, Maurer K, Linden DEJ. 2010. Specialization in the default mode: Task-induced brain deactivations dissociate between visual working memory and attention. Human Brain Mapping. 31:126-139.

McKeown MJ, Sejnowski TJ. 1998. Independent component analysis of fMRI data: examining the assumptions. Human Brain Mapping. 6:368-72.

Mesulam M. 1990. Large-scale neurocognitive networks and distributed processing for attention, language, and memory. Annals of Neurology. 28:597-613.

Mickley Steinmetz KR, Kensinger EA. 2009. The effects of valence and arousal on the neural activity leading to subsequent memory. Psychophysiology. 46:1190-9.

Minzenberg MJ, Laird AR, Thelen S, Carter CS, Glahn DC. 2009. Meta-analysis of 41 functional neuroimaging studies of executive function in schizophrenia. Archives of General Psychiatry. 66:811-822.

Moscovitch M, Cabeza R, Winocur G, Nadel L. 2016. Episodic Memory and Beyond: The Hippocampus and Neocortex in Transformation. Annual Review of Psychology. 67:105134.

Muhle-Karbe PS, Derrfuss J, Lynn MT, Neubert FX, Fox PT, Brass M, Eickhoff SB. 2015. Co-activation-based parcellation of the lateral prefrontal cortex delineates the inferior frontal junction area. Cerebral Cortex. 26:2225-2241.

Nagahama Y, Okada T, Katsumi Y, Hayashi T, Yamauchi H, Oyanagi C, Konishi J, Fukuyama H, Shibasaki H. 2001. Dissociable mechanisms of attentional control within the human prefrontal cortex. Cerebral Cortex. 11:85-92.

Ngo GH, Eickhoff SB, Fox PT, Yeo BTT. 2016. Collapsed variational Bayesian inference of the author-topic model: application to large-scale coordinate-based meta-analysis. Proceedings of the 2016 International Workshop in Pattern Recognition in Neuroimaging (PRNI).

Omori M, Yamada H, Murata T, Sadato N, Tanaka M, Ishii Y, Isaki K, Yonekura Y. 1999. Neuronal substrates participating in attentional set-shifting of rules for visually guided motor selection: a functional magnetic resonance imaging investigation. Neuroscience Research. 33:317-323.

Pardo JV, Pardo PJ, Janer KW, Raichle ME. 1990. The anterior cingulate cortex mediates processing selection in the Stroop attentional conflict paradigm. Proceedings of the National Academy of Sciences. 87:256-259.

Pesenti M, Thioux M, Seron X, De Volder A. 2000. Neuroanatomical substrates of Arabic number processing, numerical comparison, and simple addition: A PET study. Journal of Cognitive Neuroscience. 12:461-479.

Peters J, Büchel C. 2010. Episodic future thinking reduces reward delay discounting through an enhancement of prefrontal-mediotemporal interactions. Neuron. 66:138-148.

Poeppel D, Guillemin A, Thompson J, Fritz J, Bavelier D, Braun AR. 2004. Auditory lexical decision, categorical perception, and FM direction discrimination differentially engage left and right auditory cortex. Neuropsychologia. 42:183-200.

Poldrack RA, Mumford JA, Schonberg T, Kalar D, Barman B, Yarkoni T. 2012. Discovering relations between mind, brain, and mental disorders using topic mapping. PLoS Comput 
Biol. 8:e1002707.

Poldrack RA, Wagner AD, Prull MW, Desmond JE, Glover GH, Gabrieli JDE. 1999. Functional specialization for semantic and phonological processing in the left inferior prefrontal cortex. Neuroimage. 10:15-35.

Poldrack RA, Yarkoni T. 2016. From brain maps to cognitive ontologies: informatics and the search for mental structure. Annual Review of Psychology. 67:587-612.

Poldrack RA. 2006. Can cognitive processes be inferred from neuroimaging data?. Trends in Cognitive Sciences. 10:59-63.

Poline J, Breeze JL, Ghosh SS, Gorgolewski K, Halchenko YO, Hanke M, Helmer KG, Marcus DS, Poldrack RA, Schwartz Y, others. 2012. Data sharing in neuroimaging research. Frontiers in Neuroinformatics. 6:9.

Pompei F, Jogia J, Tatarelli R, Girardi P, Rubia K, Kumari V, Frangou S. 2011. Familial and disease specific abnormalities in the neural correlates of the Stroop Task in Bipolar Disorder. Neuroimage. 56:1677-1684.

Prebble SC, Addis DR, Tippett LJ. 2013. Autobiographical memory and sense of self.. Psychological bulletin. 139:815.

Race E, Keane MM, Verfaellie M. 2011. Medial temporal lobe damage causes deficits in episodic memory and episodic future thinking not attributable to deficits in narrative construction. Journal of Neuroscience. 31:10262-10269.

Raichle ME, MacLeod AM, Snyder AZ, Powers WJ, Gusnard DA, Shulman GL. 2001. A default mode of brain function. Proceedings of the National Academy of Sciences. 98:676-682.

Ravnkilde B, Videbech P, Rosenberg R, Gjedde A, Gade A. 2002. Putative tests of frontal lobe function: a PET-study of brain activation during Stroop's Test and verbal fluency. Journal of Clinical and Experimental Neuropsychology. 24:534-47.

Reid AT, Bzdok D, Genon S, Langner R, Müller VI, Eickhoff CR, Hoffstaedter F, Cieslik, E.-C.; Fox PT, Laird AR, others. 2016. ANIMA: A data-sharing initiative for neuroimaging meta-analyses. Neuroimage. 124:1245-1253.

Reid AT, Bzdok D, Langner R, Fox PT, Laird AR, Amunts K, Eickhoff SB, Eickhoff CR. 2016. Multimodal connectivity mapping of the human left anterior and posterior lateral prefrontal cortex. Brain Structure and Function. 221:2589-2605.

Rivera SM, Menon V, White CD, Glaser B, Reiss AL. 2002. Functional brain activation during arithmetic processing in females with fragile $\mathrm{X}$ Syndrome is related to FMR1 protein expression. Human Brain Mapping. 16:206-18.

Robinson JL, Laird AR, Glahn DC, Lovallo WR, Fox PT. 2010. Metaanalytic connectivity modeling: delineating the functional connectivity of the human amygdala. Human Brain Mapping. 31:173-184.

Rosen-Zvi M, Chemudugunta C, Griffiths T, Smyth P, Steyvers M. 2010. Learning authortopic models from text corpora. ACM Transactions on Information Systems (TOIS). 28:4.

Rosenbaum RS, Gilboa A, Levine B, Winocur G, Moscovitch M. 2009. Amnesia as an impairment of detail generation and binding: evidence from personal, fictional, and semantic narratives in KC. Neuropsychologia. 47:2181-2187.

Rosenbaum RS, Stuss DT, Levine B, Tulving E. 2007. Theory of mind is independent of episodic memory. Science. 318:1257-1257.

Rottschy C, Langner R, Dogan I, Reetz K, Laird AR, Schulz JB, Fox PT, Eickhoff SB. 2012. Modelling neural correlates of working memory: a coordinate-based meta-analysis. Neuroimage. 60:830-846.

Salimi-Khorshidi G, Smith SM, Keltner JR, Wager TD, Nichols TE. 2009. Meta-analysis of neuroimaging data: a comparison of image-based and coordinate-based pooling of 
studies. Neuroimage.45:810-823.

Salomon R, Levy DR, Malach R. 2014. Deconstructing the default: Cortical subdivision of the default mode/intrinsic system during self-related processing. Human Brain Mapping. 35:1491-1502.

Schacter DL, Addis DR, Buckner RL. 2007. Remembering the past to imagine the future: the prospective brain. Nature Reviews Neuroscience. 8:657-661.

Schwarz G, others. 1978. Estimating the dimension of a model. The Annals of Statistics. 6:461-464.

Seeley WW, Menon V, Schatzberg AF, Keller J, Glover GH, Kenna H, Reiss AL, Greicius MD. 2007. Dissociable intrinsic connectivity networks for salience processing and executive control. Journal of Neuroscience. 27:2349-2356.

Seghier ML, Price CJ. 2012. Functional heterogeneity within the default network during semantic processing and speech production. Frontiers in Psychology. 3:281.

Sestieri C, Corbetta M, Romani GL, Shulman GL. 2011. Episodic memory retrieval, parietal cortex, and the default mode network: functional and topographic analyses. Journal of Neuroscience. 31:4407-4420.

Sevinc G, Spreng RN. 2014. Contextual and perceptual brain processes underlying moral cognition: a quantitative meta-analysis of moral reasoning and moral emotions. PloS one. 9:e87427.

Shackman AJ, Salomons TV, Slagter HA, Fox AS, Winter JJ, Davidson RJ. 2011. The integration of negative affect, pain and cognitive control in the cingulate cortex. Nature Reviews Neuroscience. 12:154-167.

Shulman GL, Fiez JA, Corbetta M, Buckner RL, Miezin FM, Raichle ME, Petersen SE. 1997. Common blood flow changes across visual tasks: II. Decreases in cerebral cortex. Journal of Cognitive Neuroscience. 9:648-663.

Simões-Franklin C, Hester R, Shpaner M, Foxe JJ, Garavan H. 2010. Executive function and error detection: the effect of motivation on cingulate and ventral striatum activity. Human Brain Mapping. 31:458-469.

Slaughter V, Peterson CC, Mackintosh E. 2007. Mind what mother says: Narrative input and theory of mind in typical children and those on the autism spectrum. Child Development. 78:839-858.

Small GW, Moody TD, Siddarth P, Bookheimer SY. 2009. Your brain on Google: patterns of cerebral activation during internet searching. The American Journal of Geriatric Psychiatry. 17:116-126.

Smallwood J. 2013. Distinguishing how from why the mind wanders: a process-occurrence framework for self-generated mental activity. Psychological Bulletin. 139:519

Smallwood J, Schooler JW, Turk DJ, Cunningham SJ, Burns P, Macrae CN. 2011. Selfreflection and the temporal focus of the wandering mind. Consciousness and Cognition. 20:1120-1126.

Smith SM, Fox PT, Miller KL, Glahn DC, Fox PM, Mackay CE, Filippini N, Watkins KE, Toro R, Laird AR, others. 2009. Correspondence of the brains functional architecture during activation and rest. Proceedings of the National Academy of Sciences. 106:13040-13045.

Sneve MH, Magnussen S, Alnæs D, Endestad T, D’Esposito M. 2013. Top-down modulation from inferior frontal junction to FEFs and intraparietal sulcus during short-term memory for visual features. Journal of Cognitive Neuroscience. 25:1944-1956.

Spaniol J, Davidson PSR, Kim ASN, Han H, Moscovitch M, Grady CL. 2009. Event-related fMRI studies of episodic encoding and retrieval: meta-analyses using activation likelihood estimation. Neuropsychologia. 47:1765-1779.

Spreng RN, Mar RA, Kim ASN. 2009. The common neural basis of autobiographical 
memory, prospection, navigation, theory of mind, and the default mode: a quantitative meta-analysis. Journal of Cognitive Neuroscience. 21:489-510.

Spreng RN, Andrews-Hanna JR. 2015. The default network and social cognition. Brain Mapping: An Encyclopedic Reference. 165-169

Sylvester CC, Wager TD, Lacey SC, Hernandez L, Nichols TE, Smith EE, Jonides J. 2003. Switching attention and resolving interference: fMRI measures of executive functions. Neuropsychologia. 41:357-370.

Teh YW, Newman D, Welling M. 2006. A collapsed variational Bayesian inference algorithm for latent Dirichlet allocation. NIPS. 6:1378-1385.

Thompson-Schill SL, Aguirre GK, Desposito M, Farah MJ. 1999. A neural basis for category and modality specificity of semantic knowledge. Neuropsychologia. 37:671-6.

Toro R, Fox PT, Paus T. 2008. Functional coactivation map of the human brain. Cerebral Cortex. 18:2553-2559.

Turkeltaub PE, Eden GF, Jones KM, Zeffiro TA. 2002. Meta-analysis of the functional neuroanatomy of single-word reading: method and validation. Neuroimage. 16:765-780.

Turkeltaub PE, Eickhoff SB, Laird AR, Fox M, Wiener M, Fox P. 2012. Minimizing withinexperiment and within-group effects in activation likelihood estimation meta-analyses. Human Brain Mapping. 33:1-13.

Tyler LK, Stamatakis EA, Post B, Randall B, Marslen-Wilson W. 2005. Temporal and frontal systems in speech comprehension: an fMRI study of past tense processing. Neuropsychologia. 43:1963-1974.

Uddin LQ, Clare Kelly AM, Biswal BB, Xavier Castellanos F, Milham MP. 2009. Functional connectivity of default mode network components: correlation, anticorrelation, and causality. Human Brain Mapping. 30:625-637.

Uddin LQ. 2015. Salience processing and insular cortical function and dysfunction. Nature Reviews Neuroscience. 16:55.

Van Essen DC, Smith SM, Barch DM, Behrens TEJ, Yacoub E, Ugurbil K, Consortium, WU-Minn HCP Consortium, others. 2013. The WU-Minn human connectome project: an overview. Neuroimage. 80:62-79.

Van Overwalle F. 2009. Social cognition and the brain: a meta-analysis. Human Brain Mapping. 30:829-858.

Wager TD, Lindquist MA, Nichols TE, Kober H, Van Snellenberg JX. 2009. Evaluating the consistency and specificity of neuroimaging data using meta-analysis. Neuroimage. 45:S210-S221.

Wager TD, Phan KL, Liberzon I, Taylor SF. 2003. Valence, gender, and lateralization of functional brain anatomy in emotion: a meta-analysis of findings from neuroimaging. Neuroimage. 19:513-531.

Wagner AD, Paré-Blagoev EJ, Clark J, Poldrack RA. 2001. Recovering meaning: left prefrontal cortex guides controlled semantic retrieval. Neuron. 31:329-338.

Wagner RK, Torgesen JK. 1987. The nature of phonological processing and its causal role in the acquisition of reading skills. Psychological Bulletin. 101:192.

Weiss EM, Golaszewski S, Mottaghy FM, Hofer A, Hausmann A, Kemmler G, Kremser C, Brinkhoff C, Felber SR, Fleischhacker WW. 2003. Brain activation patterns during a selective attention test - a functional MRI study in healthy volunteers and patients with schizophrenia. Psychiatry Research: Neuroimaging. 123:1-15.

Worsley KJ, Marrett S, Neelin P, Vandal AC, Friston KJ, Evans AC, others. 1996. A unified statistical approach for determining significant signals in images of cerebral activation. Human Brain Mapping. 4:58-73.

Xu B, Grafman J, Gaillard WD, Ishii K, Vega-Bermudez F, Pietrini P, Reeves-Tyer P, DiCamillo P, Theodore W. 2001. Conjoint and extended neural networks for the 
computation of speech codes: the neural basis of selective impairment in reading words and pseudowords. Cerebral Cortex. 11:267-277.

Yarkoni T, Poldrack RA, Nichols TE, Van Essen DC, Wager TD. 2011. Large-scale automated synthesis of human functional neuroimaging data. Nature Methods. 8:665670.

Yeo BTT, Krienen FM, Chee MWL, Buckner RL. 2014. Estimates of segregation and overlap of functional connectivity networks in the human cerebral cortex. Neuroimage. 88:212-227.

Yeo BTT, Krienen FM, Eickhoff SB, Yaakub SN, Fox PT, Buckner RL, Asplund CL, Chee MWL. 2015. Functional specialization and flexibility in human association cortex. Cerebral Cortex. 25:3654-3672.

Zago L, Pesenti M, Mellet E, Crivello F, Mazoyer B, Tzourio-Mazoyer N. 2001. Neural correlates of simple and complex mental calculation. Neuroimage. 13.

Zandbelt BB, van Buuren M, Kahn RS, Vink M. 2011. Reduced proactive inhibition in schizophrenia is related to corticostriatal dysfunction and poor working memory. Biological Psychiatry. 70:1151-1158.

Zanto TP, Rubens MT, Bollinger J, Gazzaley A. 2010. Top-down modulation of visual feature processing: the role of the inferior frontal junction. Neuroimage. 53:736-745. 


\section{Appendix}

\section{Pseudo-code}

(1) Read the input activation foci and task labels

(2) Initialize the model's hyperparameters

(3) Repeat for $\mathrm{N}=1000$ re-initializations

(a) Initialize the variational parameters $\phi$

(b) Update the variational parameters $\phi$ (Eq. 14 in Supplemental S1)

- Approximate $E_{q}\left(N_{. . \cdot \cdot \cdot}^{-e f}\right)$ and $\operatorname{Var}_{q}\left(N_{. \cdot c^{\circ} \cdot}^{-e f}\right)$

- Approximate $E_{q}\left(N_{. \cdot c v_{e f}}^{-e f}\right)$ and $\operatorname{Var}_{q}\left(N_{. \cdot c v_{e f}}^{-e f}\right)$

- Approximate $E_{q}\left(N_{\cdot \cdot \cdot \cdot}^{-e f}\right)$ and $\operatorname{Var}_{q}\left(N_{\cdot \cdot \cdot *}^{-e f}\right)$

- Approximate $E_{q}\left(N_{\cdot t c^{\cdot}}^{-e f}\right)$ and $\operatorname{Var}_{q}\left(N_{\cdot t c^{\cdot}}^{-e f}\right)$

- Update the variational parameters $\phi$ (Eq. 14 in Supplemental S1)

- Recompute the variational log likelihood. If it converges, go to step (3c), otherwise repeat from $\operatorname{step}(3 b)$

(c) Update the model parameters $\theta_{t c}$ and $\beta_{c v}$ (Eq. 16 and 17 in Supplemental S1)

\section{Function}

CBIG_AuthorTopic_PreprocessIn put

CBIG_AuthorTopic_SetupParame ters

CBIG_AuthorTopic_InitializePara $\mathrm{ms}$

CBIG_AuthorTopic_ComputeVar iationalTerm_N_c

CBIG_AuthorTopic_ComputeVar iationalTerm_N_cv

CBIG_AuthorTopic_ComputeVar iationalTerm_N_t

CBIG_AuthorTopic_ComputeVar iationalTerm_N_tc

CBIG_AuthorTopic_EstimatePara ms

Pseudo-code of the Collapsed Variational Bayes (CVB) algorithm for estimating the authortopic model's parameters. The left column outlines the main steps of the algorithm. The right column denotes the functions in the source code that correspond to each step. The source code of the CVB algorithm and the input file of the self-generated thought dataset are available at https://github.com/ThomasYeoLab/CBIG/tree/master/stable projects/metaanalysis/Ngo2019_AuthorTopic. Note that steps (2) and (3) can be called by a single function CBIG_AuthorTopic_RunInference. 


\section{Beyond Consensus: Embracing Heterogeneity in Curated Neuroimaging Meta-Analysis}

\section{Supplemental Material}

This supplemental material is divided into Supplemental Results and Supplemental

Methods to complement Results and Methods section in the main text, respectively.

\section{Supplemental Results}

\subsection{Supplemental Result Tables}

1.1.1 Table S1. Definitions of 26 BrainMap task categories (paradigm classes) tagged to experiments activating the IFJ. Only paradigm classes tagged to at least 5 experiments were included. The definitions were extracted from BrainMap lexicon, available at http://www.brainmap.org/scribe/BrainMapLex.xls

Paradigm Class
Counting/Calculation
Cued Explicit
Recognition/Recall
Delayed Match to Sample
Emotion Induction
Encoding
Face Monitor/Discrimination
Film Viewing
Finger Tapping/Button Press
Go/No-Go
n-back

Orthographic Discrimination

Passive Listening

Passive Viewing

\section{Definition}

Count, add, subtract, multiply, or divide various stimuli (numbers, bars, dots, etc.) or solve numerical word problems.

A list of items (words, objects, textures, patterns, pictures, sounds) is presented and the subject is subsequently tested with cues to recall previously presented material.

A stimulus that is followed by a probe item after a brief delay; subject is then asked to recall if the probe item was presented before the delay.

Stimuli with emotional valence (i.e. statements, films, music, pictures) to induce effect on mood.

Memorize stimuli such as words, pictures, letters, etc.

Incidental Encoding: A task in which the subject is creating new memories without purposely knowing that memorization is the task at hand. Their memories are created thorough working in their environment and picking up information in the process.

View face passively or discriminate human faces according to their order, gender, location, emotion, or appearance, etc.

View movies or film clips.

Tap fingers or press a button in a cued/non-cued manner.

Perform a binary decision (go or no-go) on a continuous stream of stimuli.

Indicate when the current stimulus matches the one from $n$ steps earlier in the sequence. Load factor $\mathrm{n}$ can be adjusted to make the task more or less difficult.

View letters and discriminate according to some written/printed feature (i.e. uppercase/lowercase, alphabetic order, same/different spelling of words, vowel/consonant, font type/size).

Listen to auditory stimuli (speech, noise, tones, etc.) and make no response.

View visual stimuli (objects, faces, letter strings, etc.) and make no response. If the presented stimuli are faces, the experiments are co-coded with Face 
Monitor/Discrimation. If presented stimuli are words, the experiments are not coded as passive viewing but rather as Reading (Covert).

Phonological Discrimination View or listen to phonemes, syllables, or words and discriminate according to some feature of their sounds (rhyming, number of syllables, homophones, pronounceable nonwords, etc.).

Reading (Covert)

Reading (Overt)

Silently read words, pseudo-words, characters, phrases, or sentences.

Reward

Read aloud words, pseudo-words, logograms, phrases, or sentences.

Semantic

Monitor/Discrimination

Stroop-Color Word

Task Switching

A stimulus that serves the role of reinforcing a desired response.

Discriminate between the meanings of individual lexical items or to indicate if target word is semantically related to the probe word.

Name the color of the ink for a list of words (color names) printed in congruent/incongruent colors, or determine if ink color and color name are congruent/incongruent. Color-congruent stimuli: ink color and color name are the same (e.g. the word "GREEN" printed in green ink). Colorincongruent stimuli: ink color and color name differ (e.g. the word "GREEN" printed in red ink).

Tone Monitor/Discrimination Listen to tones passively or discriminate according to a sound property (i.e. order, timing, pitch, frequency, amplitude), and/or detect presence/absence of a tone.

Visual Object Identification Identify an object based on its visual attributes (e.g. shape, color, viewing angle), or detect/discriminate changes on the object's visual properties (e.g. size, illumination, position, relation between parts).

Visuospatial Attention

Make cued/noncued shifts of visual attention to a particular spatial location in the visual field. Responses can be overt (with eye movement to target location) or covert (fixating on a central target while paying attention to spatial location changes of peripheral target).

Wisconsin Card Sorting Test Sort cards into groups based on some dimension (i.e.: color, form, or number) that is changed intermittently, and requires subject to identify a new correct group dimension.

Word Generation (Covert) Semantic: Listen to or view nouns and silently generate an associated verb, or view a category and silently generate as many exemplars as possible.

Orthographic: Listen to or view a letter and silently generate as many words as possible that start with that letter.

Phonologic: Listen to or view a word and silently generate words that rhyme.

Word Generation (Overt)

Semantic: Listen to or view nouns and overtly generate an associated verb, or view a category and overtly generate as many exemplars as possible.

Orthographic: Listen to or view a letter and overtly generate as many words as possible that start with that letter.

Phonologic: Listen to or view a word and overtly generate words that rhyme. 
1.1.2 Table S2. Top 5 tasks with the highest probabilities of recruiting a co-activation pattern involving the IFJ

\section{Co-activation Pattern C1}

\begin{tabular}{lc}
\hline Task & Pr (C1 | Task) \\
Semantic Monitor/Discrimination & 0.51 \\
Reading (Covert) & 0.50 \\
Phonological Discrimination & 0.49 \\
Film Viewing & 0.45 \\
Word Generation (Overt) & 0.42
\end{tabular}

\section{Co-activation Pattern C2}

Task

Counting/Calculation

Task Switching

Wisconsin Card Sorting Task

Stroop-Color Word

Delayed Match to Sample
$\operatorname{Pr}$ (C2 | Task)

0.63

0.60

0.60

0.49

0.46

Co-activation Pattern C3

Task

Go/ No-Go

Encoding

Word Generation (Overt)

Reward

Reading (Overt)
$\operatorname{Pr}(\mathrm{C} 3$ | Task $)$

0.54

0.44

0.42

0.41

0.36 
1.1.3 Table S3-A. Experiments with the highest probabilities of recruiting co-activation pattern $\mathrm{C} 1$ of IFJ

Semantic Monitor/Discrimination

\begin{tabular}{|c|c|c|c|c|c|}
\hline PMID & Author & Title & $\operatorname{Exp}$ & Contrast & Pr \\
\hline 10390028 & $\begin{array}{l}\text { Thompson } \\
\text {-Schill } \\
1999\end{array}$ & $\begin{array}{l}\text { A neural basis for category and } \\
\text { modality specificity of semantic } \\
\text { knowledge }\end{array}$ & 3 & $\begin{array}{l}\text { Retrieval of visual } \\
\text { knowledge about nonliving } \\
\text { things - baseline }\end{array}$ & 0.81 \\
\hline 14644105 & $\begin{array}{l}\text { Poeppel } \\
2004\end{array}$ & $\begin{array}{l}\text { Auditory lexical decision, } \\
\text { categorical perception, and FM } \\
\text { direction discrimination } \\
\text { differentially engage left and right } \\
\text { auditory cortex }\end{array}$ & 5 & $\begin{array}{l}\text { Lexical decision } \\
\text { of phonologically permissibl } \\
\text { e targets vs. frequency- } \\
\text { modulated signals }\end{array}$ & 0.80 \\
\hline 11502262 & $\begin{array}{l}\text { Wagner } \\
2001\end{array}$ & $\begin{array}{l}\text { Recovering meaning: Left prefrontal } \\
\text { cortex guides controlled semantic } \\
\text { retrieval }\end{array}$ & 4 & $\begin{array}{l}4 \text { target items }>2 \text { target } \\
\text { items (weak cue-correct } \\
\text { response association) }\end{array}$ & 0.79 \\
\hline \multicolumn{6}{|c|}{ Reading (Covert) } \\
\hline 17110132 & $\begin{array}{l}\text { Jobard } \\
2007\end{array}$ & $\begin{array}{l}\text { Impact of modality and linguistic } \\
\text { complexity during reading and } \\
\text { listening tasks }\end{array}$ & 3 & $\begin{array}{l}\text { Conjunction of reading and } \\
\text { listening irrespective of the } \\
\text { linguistic complexity }\end{array}$ & 0.87 \\
\hline 19155745 & Small 2009 & $\begin{array}{l}\text { Your brain on Google: patterns of } \\
\text { cerebral activation during internet } \\
\text { searching }\end{array}$ & 3 & $\begin{array}{l}\text { Internet task in Internet- } \\
\text { savvy group }\end{array}$ & 0.72 \\
\hline 19155745 & Small 2009 & $\begin{array}{l}\text { Your brain on Google: patterns of } \\
\text { cerebral activation during internet } \\
\text { searching }\end{array}$ & 2 & $\begin{array}{l}\text { Reading task in Internet- } \\
\text { naïve group }\end{array}$ & 0.64 \\
\hline
\end{tabular}

Phonological Discrimination

\begin{tabular}{|c|c|c|c|c|c|}
\hline 11230098 & Xu 2001 & $\begin{array}{l}\text { Conjoint and extended neural } \\
\text { networks for the computation of } \\
\text { speech codes: The neural basis of } \\
\text { selective impairment in reading } \\
\text { words and pseudowords }\end{array}$ & 4 & $\begin{array}{l}\text { Real-word rhyming - color- } \\
\text { matching with letters } \\
\text { (Group) }\end{array}$ & 0.86 \\
\hline 7922456 & $\begin{array}{l}\text { Démonet } \\
1994\end{array}$ & $\begin{array}{l}\text { A PET study of cognitive strategies } \\
\text { in normal subjects during language } \\
\text { tasks. Influence of phonetic } \\
\text { ambiguity and sequence processing } \\
\text { on phoneme monitoring }\end{array}$ & 4 & $\begin{array}{l}\text { Phoneme monitoring of } \\
\text { perceptually ambiguous } \\
\text { sequence of non-words - } \\
\text { monitoring pure tones }\end{array}$ & 0.85 \\
\hline 11230098 & Xu 2001 & $\begin{array}{l}\text { Conjoint and extended neural } \\
\text { networks for the computation of } \\
\text { speech codes: The neural basis of }\end{array}$ & 7 & $\begin{array}{l}\text { Alternate-case rhyming - } \\
\text { color-matching with letters } \\
\text { (Group) }\end{array}$ & 0.83 \\
\hline
\end{tabular}
selective impairment in reading words and pseudowords

Each row shows one of the top 3 experiments with the highest probabilities of recruiting co-activation pattern $\mathrm{C} 1$ of IFJ and employing one of the top 3 tasks with the highest probabilities of recruiting C1, namely "Semantic Monitoring/Discrimination", "Covert Reading", and "Phonological Discrimination". The "PMID" and "Title" columns list the PubMed ID and title of each study respectively. The "Author" column lists the last name of the first author and the year of publication of each study. The "Exp" column lists the experiment's order in the respective study as reported in BrainMap. The "Contrast" column lists the experimental contrast of each experiment. The "Pr" column shows the probability that each experiment would recruit co-activation pattern $\mathrm{C} 1$. 
1.1.4 Table S3-B. Experiments with the highest probabilities of recruiting co-activation pattern $\mathrm{C} 2$ of IFJ

Counting/ Calculation

\begin{tabular}{|c|c|c|c|c|c|}
\hline PMID & Author & Title & Exp & Contrast & Pr \\
\hline 11162272 & Zago 2001 & $\begin{array}{l}\text { Neural Correlates of Simple and Complex } \\
\text { Mental Calculation }\end{array}$ & 4 & $\begin{array}{l}\text { Compute vs. retrieve } \\
\text { masked by compute vs. } \\
\text { read }\end{array}$ & 0.97 \\
\hline 11162272 & Zago 2001 & $\begin{array}{l}\text { Neural Correlates of Simple and Complex } \\
\text { Mental Calculation }\end{array}$ & 2 & Compute vs. read & 0.95 \\
\hline 12112763 & Rivera 2002 & $\begin{array}{l}\text { Functional brain activation during } \\
\text { arithmetic processing in females with } \\
\text { fragile X Syndrome is related to FMR } 1 \\
\text { protein expression }\end{array}$ & 3 & $\begin{array}{l}\text { Judge correctness of } \\
\text { arithmetic equation } \\
\text { with } 3 \text { operands } \\
\text { (unaffected) - Control }\end{array}$ & 0.81 \\
\hline \multicolumn{6}{|c|}{ Task Switching } \\
\hline 10401985 & Omori 1999 & $\begin{array}{l}\text { Neuronal substrates participating in } \\
\text { attentional set-shifting of rules for visually } \\
\text { guided motor selection: a functional } \\
\text { magnetic resonance imaging investigation }\end{array}$ & 2 & $\begin{array}{l}\text { Conjunction among } \\
\text { alternate vs. rest, tie vs. } \\
\text { rest, win vs. rest, lose } \\
\text { vs. rest }\end{array}$ & 0.83 \\
\hline 11113037 & $\begin{array}{l}\text { Nagahama } \\
2001\end{array}$ & $\begin{array}{l}\text { Dissociable mechanisms of attentional } \\
\text { control within the human prefrontal cortex }\end{array}$ & 2 & Reversal task & 0.81 \\
\hline 12457760 & $\begin{array}{l}\text { Sylvester } \\
2003\end{array}$ & $\begin{array}{l}\text { Switching attention and resolving } \\
\text { interference: fMRI measures of executive }\end{array}$ & 3 & $\begin{array}{l}\text { Counter-switching task } \\
\text { (blocked design) }\end{array}$ & 0.79 \\
\hline
\end{tabular}

Wisconsin Card Sorting Test

\begin{tabular}{llllll}
\hline 8524452 & $\begin{array}{l}\text { Berman } \\
1995\end{array}$ & $\begin{array}{l}\text { Physiological activation of a cortical } \\
\text { network during performance of the } \\
\text { Wisconsin Card Sorting Test: A positron } \\
\text { emission tomography study }\end{array}$ & 1 & $\begin{array}{l}\text { Wisconsin Card } \\
\text { Sorting Task > Control }\end{array}$ & 0.76 \\
12944506 & $\begin{array}{l}\text { Konishi } \\
2003\end{array}$ & $\begin{array}{l}\text { Transient Activation of Superior Prefrontal } \\
\text { Cortex during Inhibition of Cognitive Set }\end{array}$ & 2 & $\begin{array}{l}\text { Inhibition - Control } \\
\text { (Subjects aware of the } \\
\text { dimensional changes) }\end{array}$ & 0.66 \\
12032364 & $\begin{array}{l}\text { Konishi } \\
2002\end{array}$ & $\begin{array}{l}\text { Hemispheric asymmetry in human lateral } \\
\text { prefrontal cortex during cognitive set } \\
\text { shifting }\end{array}$ & 2 & $\begin{array}{l}\text { Event B (omitting the } \\
\text { negative feedback } \\
\text { simulus) - Event C } \\
\text { ("null" change } \\
\text { instruction) }\end{array}$ & 0.57 \\
& & & &
\end{tabular}

Each row shows one of the top 3 experiments with the highest probabilities of recruiting co-activation pattern $\mathrm{C} 2$ of IFJ and employing one of the top 3 tasks with the highest probabilities of recruiting C2, namely "Counting/Calculation", "Task Switching", and "Wisconsin Card Sorting Test". The "PMID" and "Title" columns list the PubMed ID and title of each study respectively. The "Author" column lists the last name of the first author and the year of publication of each study. The "Exp" column lists the experiment's order in the respective study as reported in BrainMap. The "Contrast" column lists the experimental contrast of each experiment. The "Pr" column shows the probability that each experiment would recruit co-activation pattern $\mathrm{C} 2$. 
1.1.5 Table S3-C. Experiments with the highest probabilities of recruiting co-activation pattern $\mathrm{C} 3$ of IFJ

\begin{tabular}{|c|c|c|c|c|c|}
\hline \multicolumn{6}{|l|}{ Go/No-Go } \\
\hline PMID & Author & Title & Exp & Contrast & Pr \\
\hline 20016103 & $\begin{array}{l}\text { Chikazoe } \\
2009\end{array}$ & $\begin{array}{l}\text { Preparation to Inhibit a Response } \\
\text { Complements Response Inhibition during } \\
\text { Performance of a Stop-Signal Task }\end{array}$ & 4 & $\begin{array}{l}\text { Disjunction analysis: } \\
\text { Stop vs. Uncertain-go } \\
\text { but not Uncertain-go vs. } \\
\text { Certain-go }\end{array}$ & 0.80 \\
\hline 19718655 & $\begin{array}{l}\text { Simoes- } \\
\text { Franklin } \\
2010\end{array}$ & $\begin{array}{l}\text { Executive function and error detection: } \\
\text { The effect of motivation on cingulate and } \\
\text { ventral striatum activity }\end{array}$ & 1 & $\begin{array}{l}\text { Go - No-go with } \\
\text { punishment }>\text { Go- No- } \\
\text { go without punishment }\end{array}$ & 0.76 \\
\hline 8864300 & $\begin{array}{l}\text { Kawashima } \\
1996\end{array}$ & $\begin{array}{l}\text { Functional anatomy of GO/NO-GO } \\
\text { discrimination and response selection-a } \\
\text { PET study in man }\end{array}$ & 14 & $\begin{array}{l}\text { Go }- \text { No-go }>\text { Control } \\
\text { Task }\end{array}$ & 0.76 \\
\hline \multicolumn{6}{|l|}{ Encoding } \\
\hline 8957565 & Kapur1996 & $\begin{array}{l}\text { The neural correlates of intentional } \\
\text { learning of verbal materials: A PET study } \\
\text { in humans }\end{array}$ & 1 & Encoding vs. Reading & 0.64 \\
\hline 19882649 & Callan 2010 & $\begin{array}{l}\text { Neural correlates of the spacing effect in } \\
\text { explicit verbal semantic encoding support } \\
\text { the deficient-processing theory }\end{array}$ & 9 & $\begin{array}{l}\text { Decreasing activity trend } \\
\text { relative to verbal } \\
\text { working memory } \\
\text { conditions in massed vs. } \\
\text { spaced presentation } \\
(\mathrm{p}<0.05)\end{array}$ & 0.61 \\
\hline 19674398 & $\begin{array}{l}\text { Mickley } \\
2009\end{array}$ & $\begin{array}{l}\text { The effects of valence and arousal on the } \\
\text { neural activity leading to subsequent } \\
\text { memory }\end{array}$ & 5 & $\begin{array}{l}\text { Remembered }- \text { forgotten } \\
\text { in positive }>\text { negative } \\
\text { arousal info }\end{array}$ & 0.57 \\
\hline \multicolumn{6}{|c|}{ Word Generation (Overt) } \\
\hline 9153677 & Baker 1997 & $\begin{array}{l}\text { The interaction between mood and } \\
\text { cognitive function studied with PET }\end{array}$ & 1 & $\begin{array}{l}\text { Increase in rCBF in } \\
\text { verbal fluency - } \\
\text { repetition task }\end{array}$ & 0.69 \\
\hline 12187466 & $\begin{array}{l}\text { Ravnkilde } \\
2002\end{array}$ & $\begin{array}{l}\text { Putative tests of frontal lobe function: A } \\
\text { PET-study of brain activation during } \\
\text { Stroop's test and verbal fluency }\end{array}$ & 2 & Verbal fluency - reading & 0.67 \\
\hline 16494687 & Desai 2006 & $\begin{array}{l}\text { FMRI of past tense processing: The } \\
\text { effects of phonological complexity and } \\
\text { task difficulty }\end{array}$ & 10 & $\begin{array}{l}\text { Generate iIrregular verbs } \\
\text { - generate regular verbs } \\
\text { (phonologically } \\
\text { matched) }\end{array}$ & 0.42 \\
\hline
\end{tabular}

Each row shows one of the top 3 experiments with the highest probabilities of recruiting co-activation pattern $\mathrm{C} 3$ of IFJ and employing one of the top 3 tasks with the highest probabilities of recruiting C3, namely "Go/No-Go", "Encoding", and "Overt Word Generation". The "PMID" and "Title" columns list the PubMed ID and title of each study respectively. The "Author" column lists the last name of the first author and the year of publication of each study. The "Exp" column lists the experiment's order in the respective study as reported in BrainMap. The "Contrast" column lists the experimental contrast of each experiment. The "Pr" column shows the probability that each experiment would recruit coactivation pattern $\mathrm{C} 3$. 


\subsection{Supplemental Result Figures}

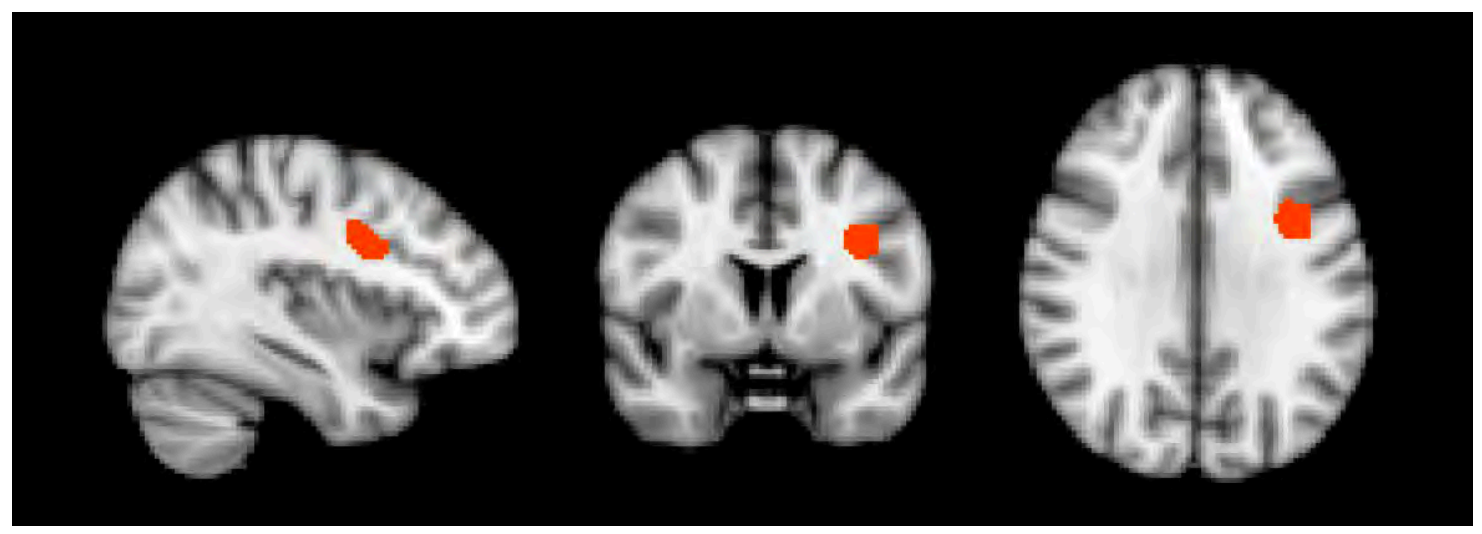

Figure S1. Illustration of the left IFJ seed region (Muhle-Karbe et al., 2015) in the sagittal, coronal and axial planes. 


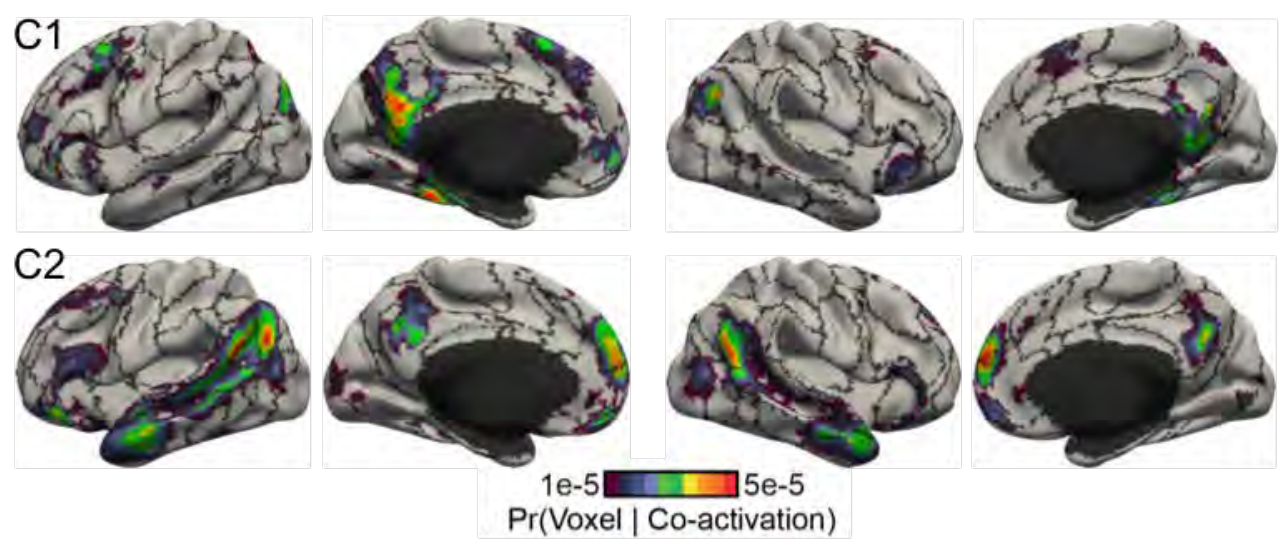

Average $\operatorname{Pr}($ voxel|component) within each of Yeo et al. 2011 17 networks

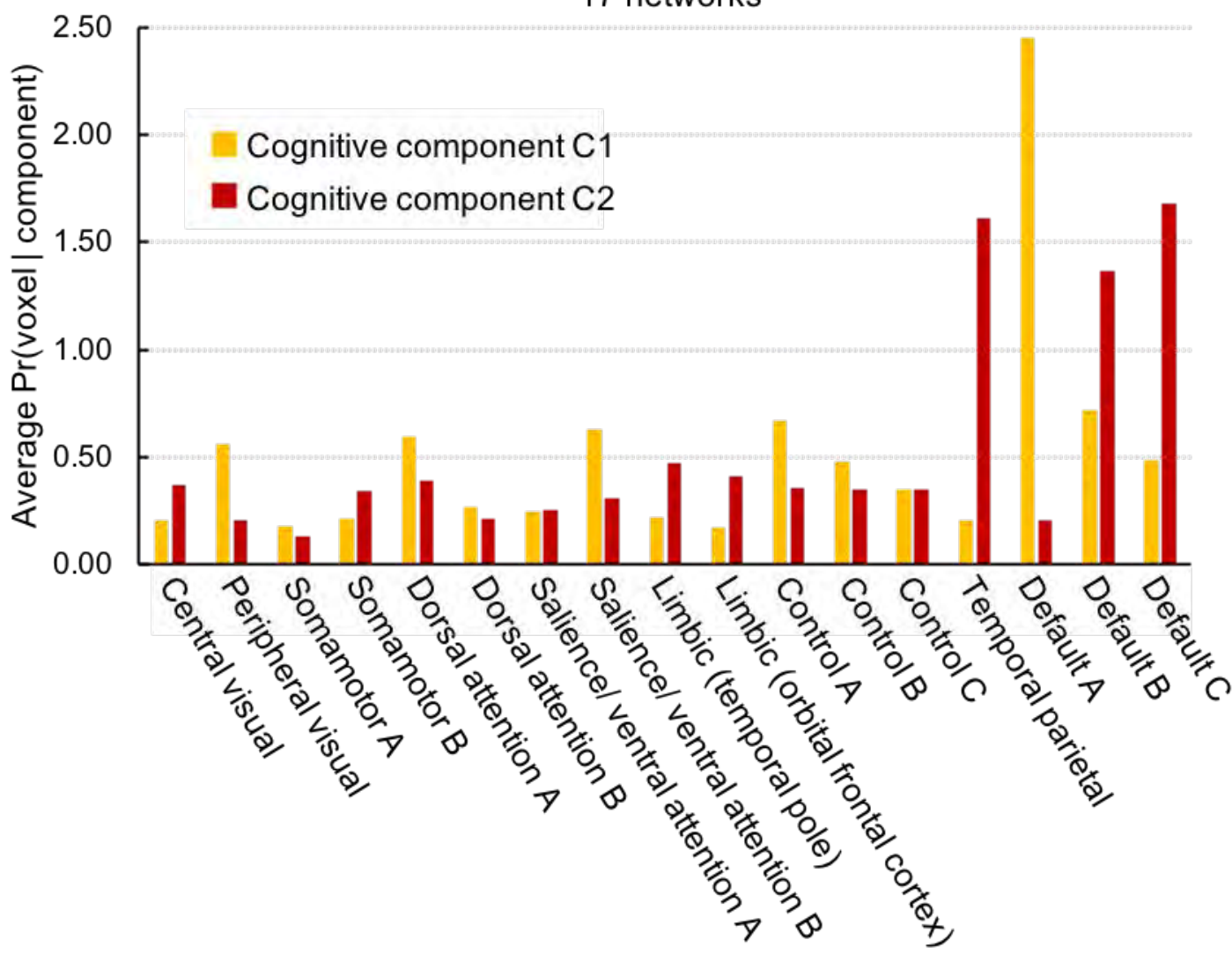

Figure S2. Comparison of the cognitive components of self-generated thought with Yeo et al. (2011) 17-network parcellation. The top figure shows the surface-based visualization of the probability of cognitive components of self-generated thought activating different brain voxels (i.e., $\operatorname{Pr}($ voxel $\mid$ component)) overlaid on top of the 17-network boundaries (black lines) from Yeo et al. (2011). The bottom bar chart shows the average $\operatorname{Pr}($ voxel $\mid$ component) of each network. The yellow and red columns correspond to cognitive components $\mathrm{C} 1$ and $\mathrm{C} 2$ respectively. 

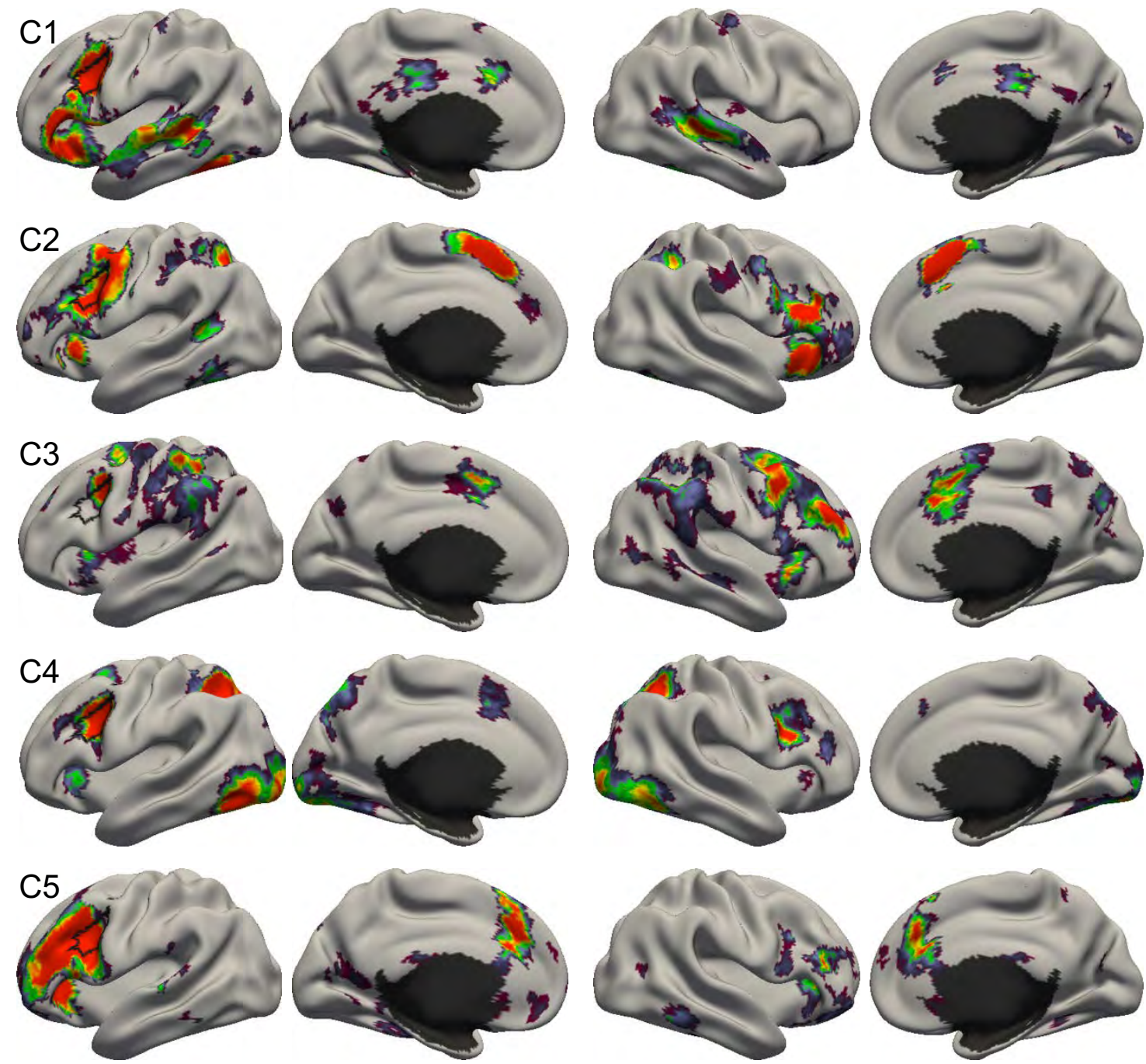

$1 \mathrm{e}-5 \square[\mathrm{e}-5$

$\operatorname{Pr}$ (Voxel | Co-activation)

Figure S3. Model estimates of 5 co-activation patterns involving the left inferior frontal junction (IFJ). Each row shows the surface-based visualization for the probability of a coactivation pattern recruiting different brain voxels (i.e., $\operatorname{Pr}($ voxel $\mid$ co-activation pattern)). Coactivation pattern $\mathrm{C} 3$ only involves a part of the left IFJ, suggesting that the 5-pattern model estimates might fractionate the seed region. 

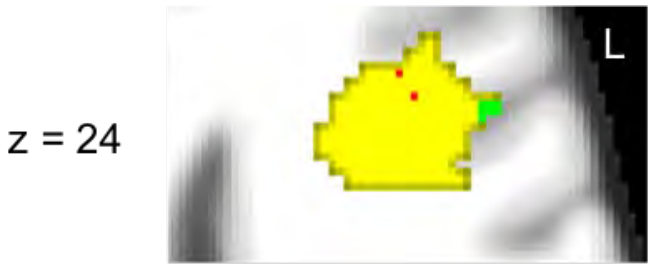

$z=26$
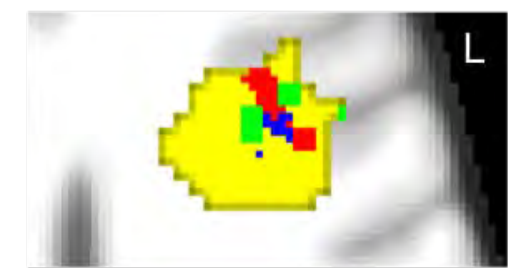

$z=28$

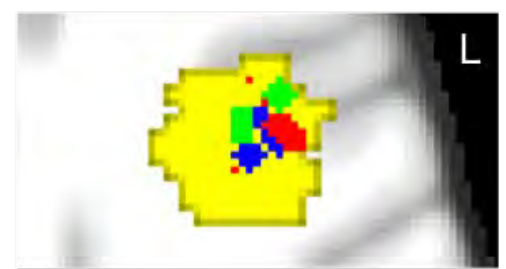

$z=30$

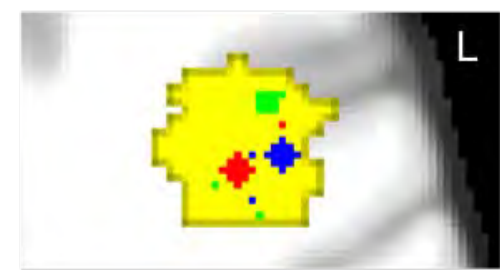

$z=32$

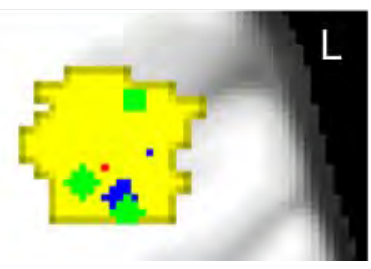

$z=34$

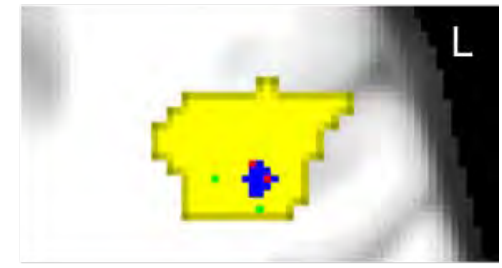

$z=36$

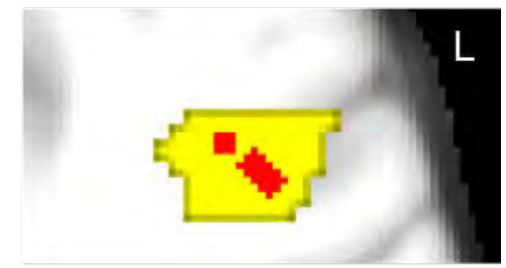

$z=38$

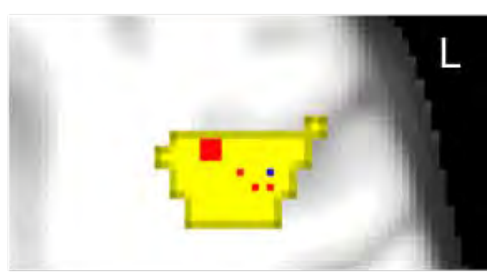

$z=40$

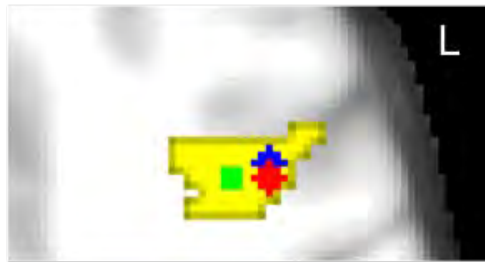

$z=42$

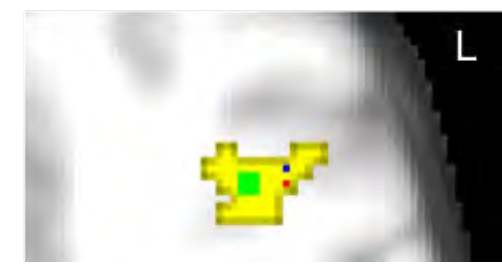

Figure S4. Volumetric slices suggesting the foci of co-activation patterns overlap within the left IFJ. Yellow indicates the left IFJ seed region. The colored dots correspond 2-mm-radius spheres centered about the activation foci reported by the top 3 experiments with the highest probabilities of recruiting IFJ co-activation patterns and employs one of the top 3 tasks with the highest probabilities of recruiting the given co-activation pattern. Blue, red and green dots correspond to the activation foci associated with co-activation patterns $\mathrm{C} 1, \mathrm{C} 2$, and $\mathrm{C} 3$, respectively. 
C1

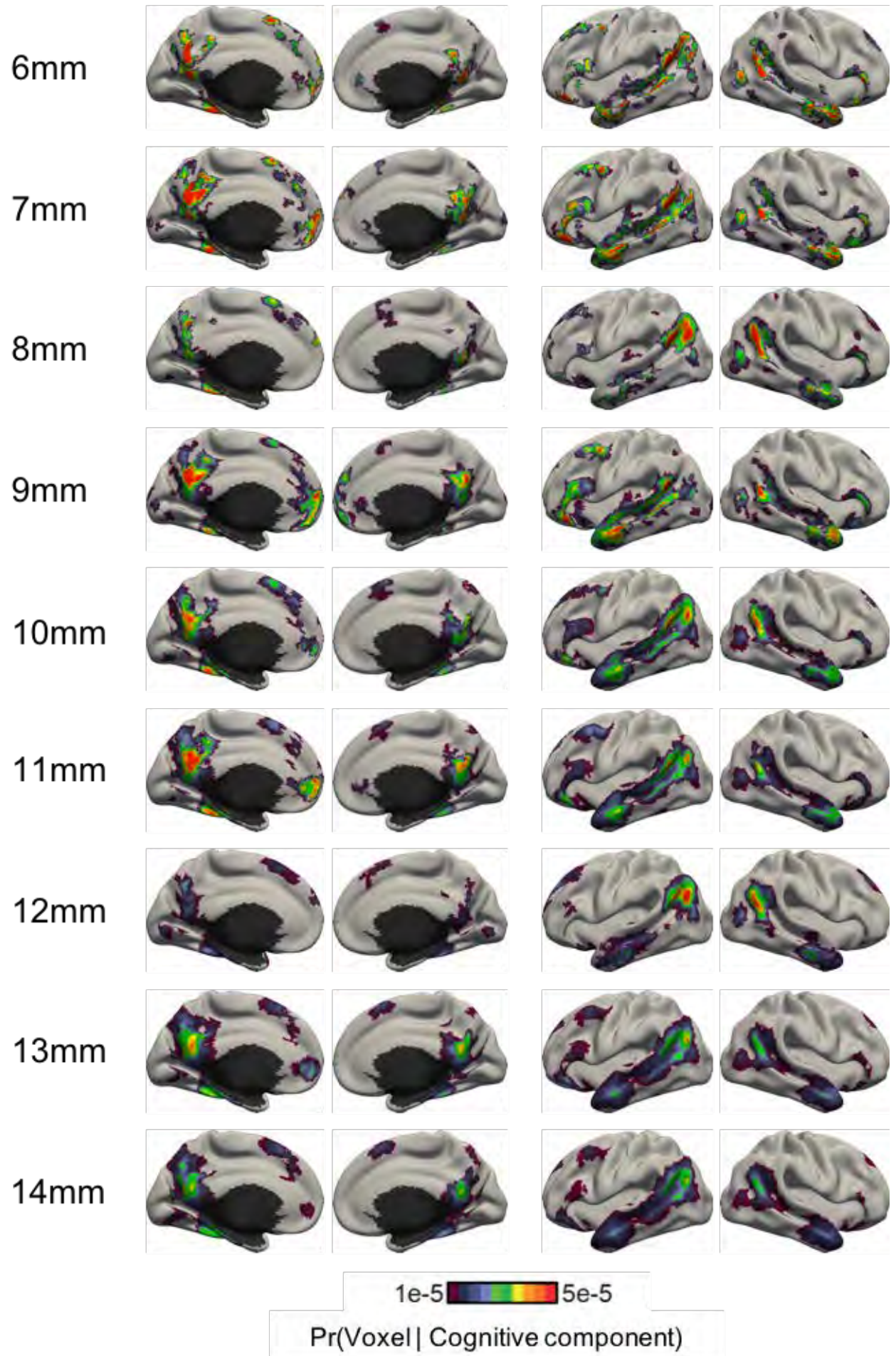

Figure S5. Cognitive components of self-generated thought estimated by the author-topic model using different radii of smoothing kernel applied to the activation foci. The smoothing radii range from $6 \mathrm{~mm}$ to $14 \mathrm{~mm}$. Cognitive components estimated with different smoothing kernel radii are similar to the components estimated with a $10 \mathrm{~mm}$-radius smoothing kernel. The average Pearsons' correlation coefficient of the cognitive components estimated with different smoothing kernel radii against those estimated with a $10 \mathrm{~mm}$-radius ranges from $0.60(6 \mathrm{~mm})$ to $0.79(14 \mathrm{~mm})$. The cognitive components estimated with a $10 \mathrm{~mm}$-radius smoothing kernel were shown in all results and analyses. 
C1

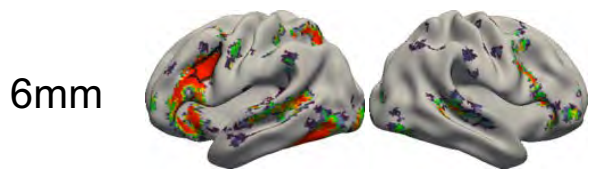

$7 \mathrm{~mm}$
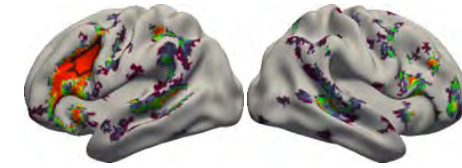

$8 \mathrm{~mm}$

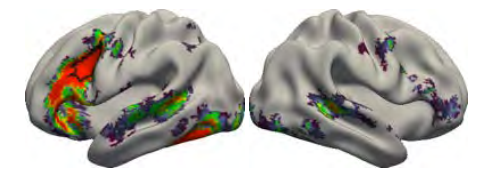

$9 \mathrm{~mm}$
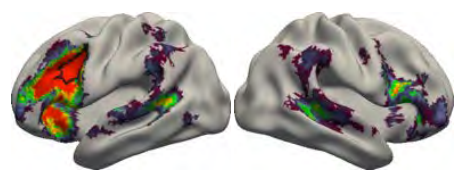

$10 \mathrm{~mm}$
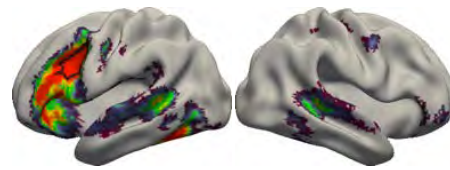

$11 \mathrm{~mm}$
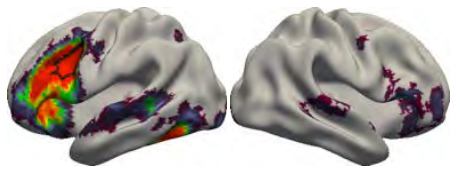

$12 \mathrm{~mm}$

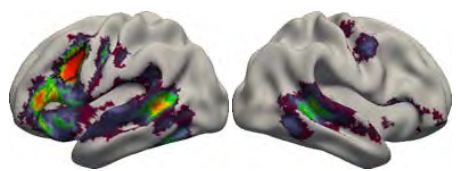

$13 \mathrm{~mm}$
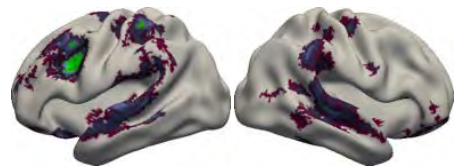

$14 \mathrm{~mm}$
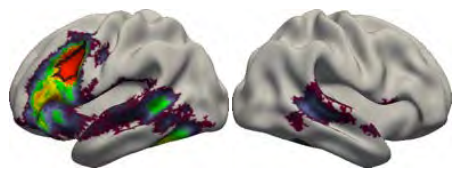

C2
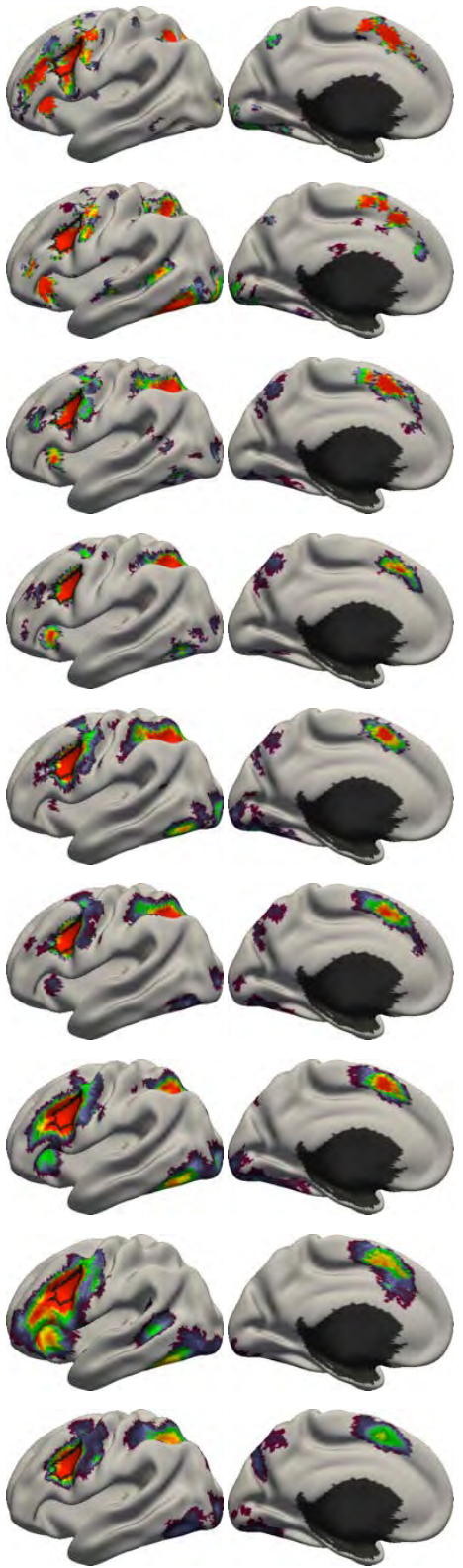

$1 \mathrm{e}-5 \square 5 \mathrm{e}-5$

$\operatorname{Pr}$ (Voxel | Co-activation)
C3
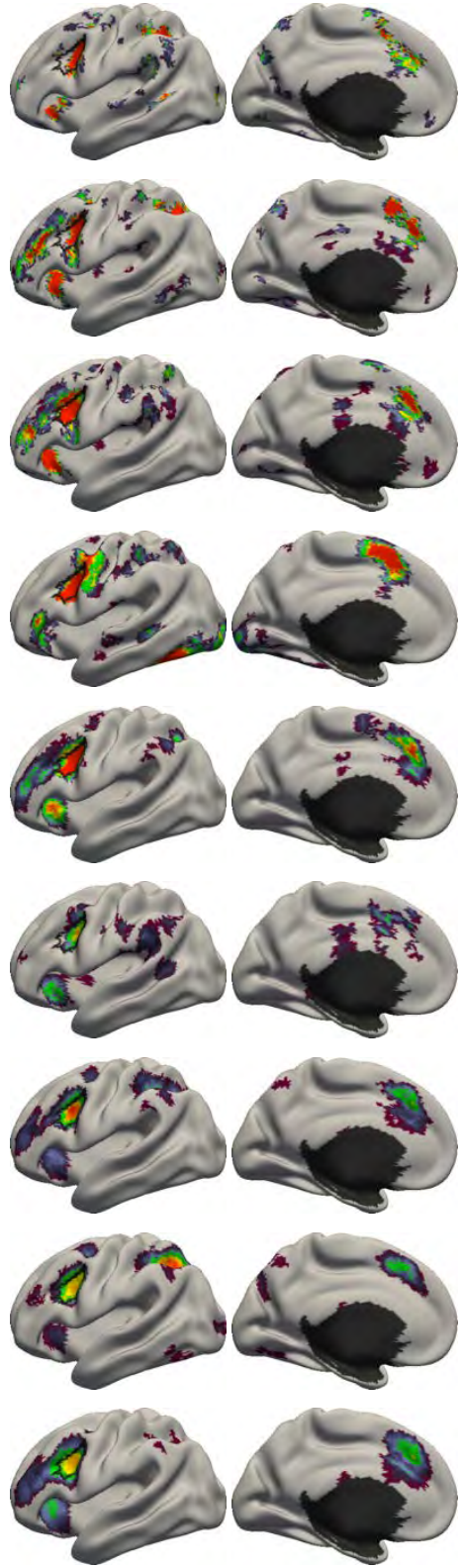

Figure S6. Co-activation patterns of the left inferior frontal junction estimated by the authortopic model across different radii of smoothing kernel applied to the input data. The smoothing radii ranged from $6 \mathrm{~mm}$ to $14 \mathrm{~mm}$. Co-activation patterns estimated with different smoothing kernel radii are similar to the co-activation patterns estimated with a $10 \mathrm{~mm}$-radius smoothing kernel. The average Pearsons' correlation coefficient of the co-activation patterns estimated with different smoothing kernel radii against those estimated with a $10 \mathrm{~mm}$-radius ranges from $0.54(6 \mathrm{~mm})$ to $0.85(14 \mathrm{~mm})$. The co-activation patterns estimated with a $10 \mathrm{~mm}$ radius smoothing kernel were shown in all results and analyses. 

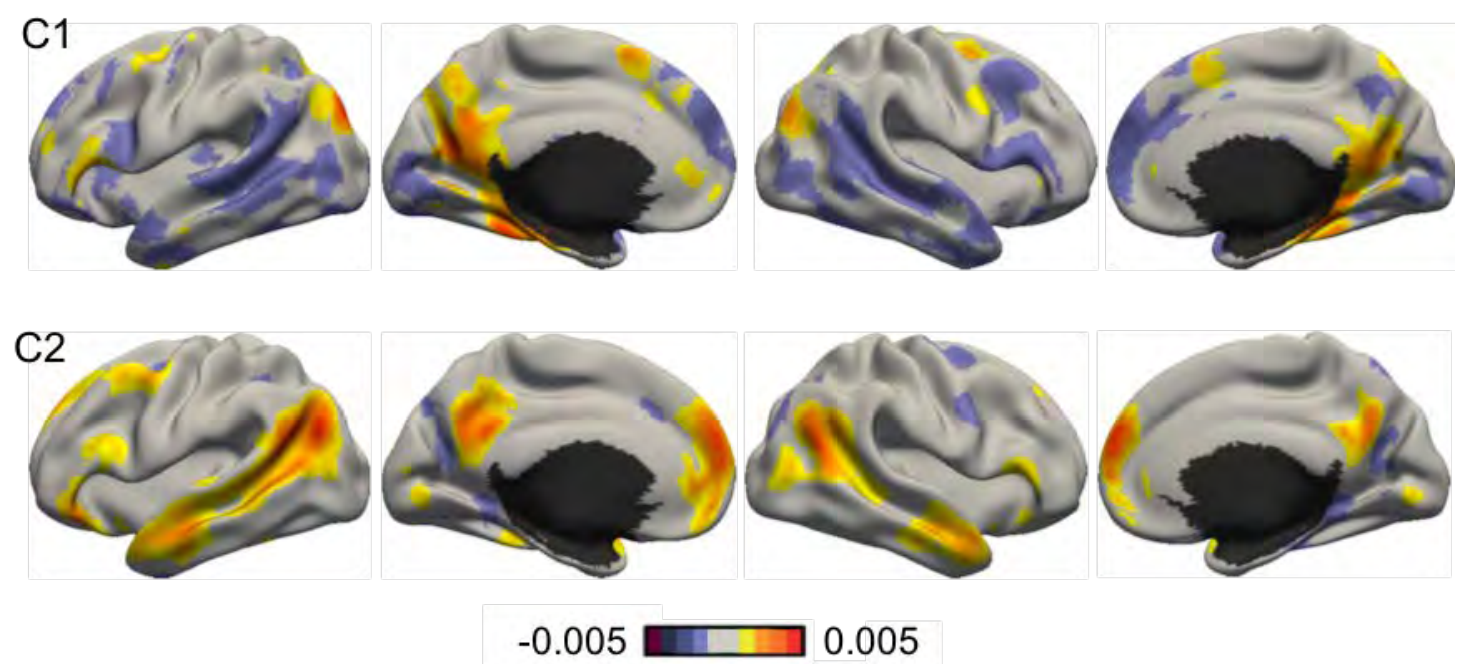

Figure S7. 2-component ICA decomposition of self-generated thought dataset. Each independent component has both positive (yellow and red) and negative values (purple and maroon). The negative values in the independent components are not directly interpretable since the input data consisted of only "activation", so it does not make sense to have "deactivation" in the component estimates. 

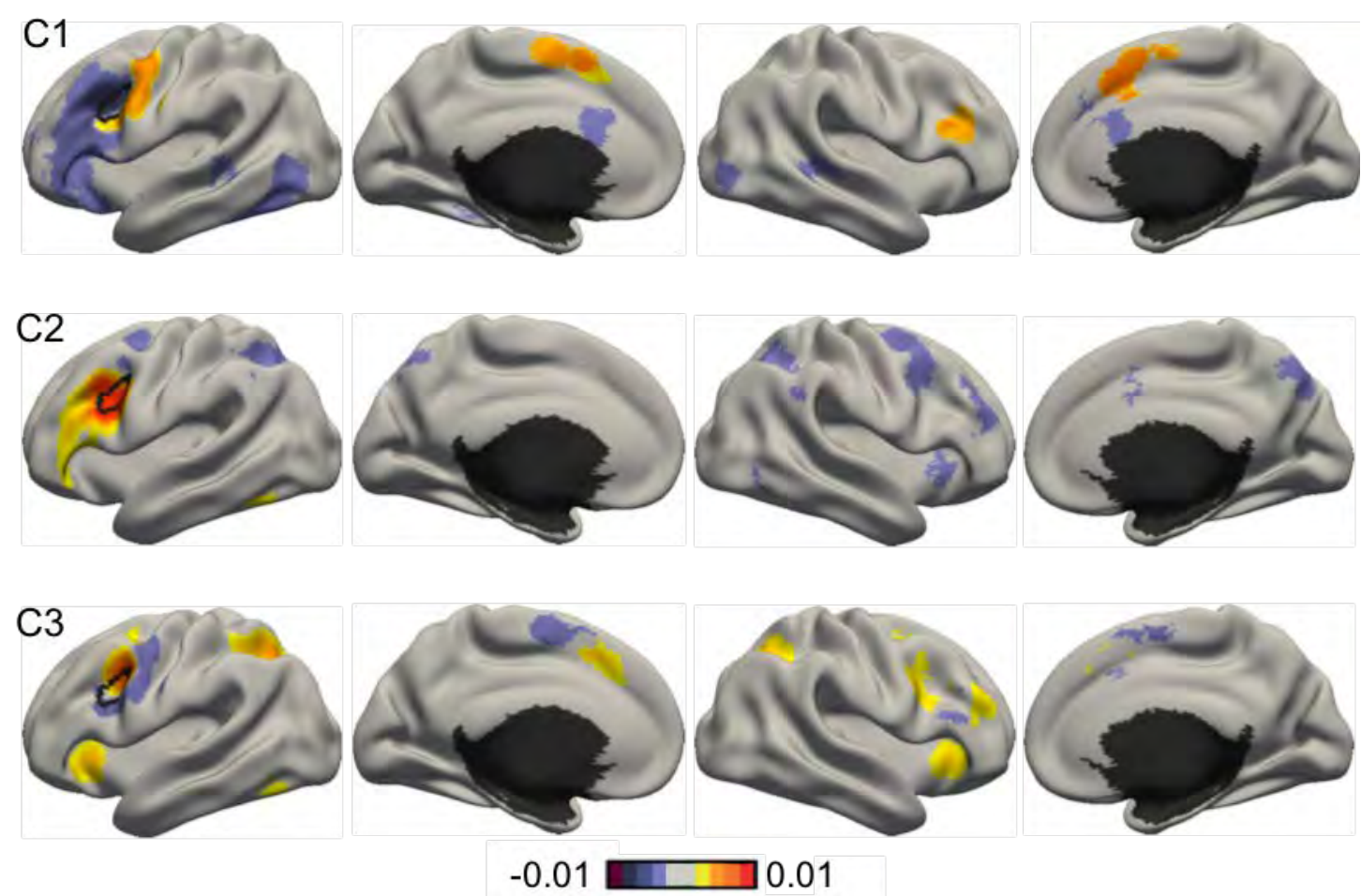

Figure S8. 3-component ICA decomposition of the left inferior frontal junction (IFJ) activation data. Each independent component has both positive (yellow and red) and negative values (purple and maroon). The negative values in independent components are not directly interpretable, since the input data consisted of only "activation", so it does not make sense to have "de-activation" in the component estimates. Component $\mathrm{C} 1$ does not engage most of the left IFJ seed region while component $\mathrm{C} 3$ has both positive and negative values within the seed region, suggesting that the ICA components might be fractionating the seed region, rather than discovering multiple co-activation patterns. 


\section{Supplemental Methods}

\section{1 (S1) Mathematical Model}

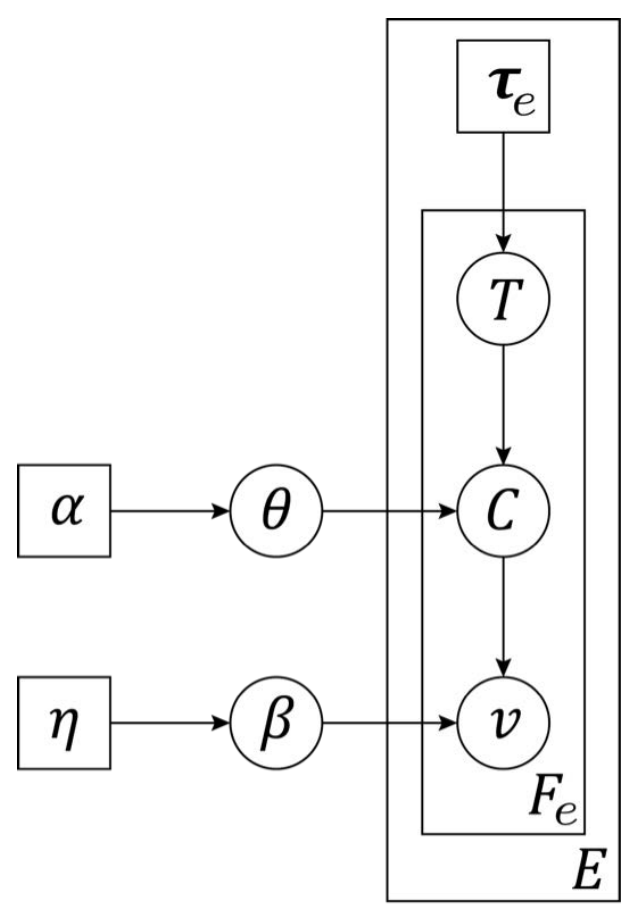

Figure S9. Formal graphical representation of the author-topic model for coordinate-based meta-analysis (Yeo et al. 2015). The circles represent random variables, while the squares represent non-random parameters. The edges represent statistical dependencies. There are $E$ experiments. The $e$-th experiment utilizes a set of behavioral tasks $\boldsymbol{\tau}_{e}$ and reports $F_{e}$ number of activated voxels. The $f$-th activated voxel has an observed location $v$, and associated with a latent (unobserved) component $C$ and a latent (unobserved) task $T \in \boldsymbol{\tau}_{e}$. The variables at the corners of the rectangles (plates) indicate the number of times the variables within the rectangles were replicated. Therefore $\boldsymbol{\tau}_{e}$ was replicated $E$ times, once for each experiment. For the $e$-th experiment, the variables $v, C$ and $T$ were replicated $F_{e}$ times, once for each activated voxel. $\theta$ denotes $\operatorname{Pr}$ (component $\mid$ task) and $\beta$ denotes $\operatorname{Pr}$ (voxel $\mid$ component). Thus $\theta$ and $\beta$ are matrices, where each row is a categorical distribution summing to one. $\alpha$ and $\eta$ are hyperparameters parameterizing the Dirichlet priors on $\theta$ and $\beta$ respectively.

Figure S9 shows the formal graphical representation of the author-topic model for coordinate-based meta-analysis. The model assumes that there are $E$ experiments. The $e$-th experiment is associated with a set of tasks $\boldsymbol{\tau}_{e}$ and an unordered set $\boldsymbol{v}_{e}$ of $F_{e}$ activated voxels. The location of the $f$-th activated voxel is denoted as $v_{e f}$, corresponding to one of $V=$ 284100 possible locations in MNI152 2mm space (Lancaster et al., 2007). The collection of activated voxels across all $E$ experiments is denoted as $\boldsymbol{v}=\left\{\boldsymbol{v}_{e}\right\}$. The collection of tasks 
across all $E$ experiments is denoted as $\boldsymbol{\tau}=\left\{\boldsymbol{\tau}_{e}\right\}$. Thus, $\{\boldsymbol{v}, \boldsymbol{\tau}\}$ are the input data for the metaanalysis.

We assume that there are $K$ cognitive components and $M$ unique tasks in the dataset. For example, $M=83$ in Yeo et al. (2015). Each task has a certain (unknown) probability of recruiting a component $\operatorname{Pr}($ component $\mid$ task). The collection of all probabilities $\operatorname{Pr}$ (component | task) is denoted by a $M \times K$ matrix $\theta$. The $t$-th row and $k$-th column of $\theta$ corresponds to the probability of the $t$-th task recruiting the $k$-th component. Each component has a certain (unknown) probability of activating a voxel pr(voxel $\mid$ component). The collection of all probabilities $\operatorname{Pr}($ voxel $\mid$ component) is denoted by a $K \times V$ matrix $\beta$. The $k$-th row and $v$-th column of $\beta$ corresponds to the probability of the $k$-th component activating the $v$-th MNI152 voxel. Symmetric Dirichlet priors with hyperparameter $\alpha$ are assumed on $\theta$, and hyperparameter $\eta$ on $\beta$.

We assume that the activated voxels of an experiment are independent and identically distributed (conditioned on knowing $\theta$ and $\beta$ ). To generate the $f$-th activated voxel $v_{e f}$ in the $e$-th experiment, a task $T_{e f}$ is sampled uniformly from the set of tasks $\boldsymbol{\tau}_{e}$ utilized by the experiment. Given task $T_{e f}$, a component $C_{e f}$ is sampled based on the probability that the task would recruit a component (corresponding to the $T_{e f}$-th row of the $\theta$ matrix). Given component $C_{e f}$, the activation location $v_{e f}$ is sampled based on the probability that the component would activate a voxel (corresponding to the $C_{e f}$-th row of the $\beta$ matrix). $T_{e f}$ and $C_{e f}$ are known as latent variables because they are not directly observed in the input data. We denote $\boldsymbol{T}=\left\{T_{e f}\right\}, \boldsymbol{C}=\left\{C_{e f}\right\}$ as the collection of latent tasks and components across all experiments and activated voxels.

Given the number of cognitive components $K$, the fixed hyperparameters $\alpha$ and $\eta$, and the activated voxels and behavioral task categories $\{\boldsymbol{v}, \boldsymbol{\tau}\}$ of all experiments, the parameters $\operatorname{Pr}($ component $\mid$ task) $\theta$, and $\operatorname{Pr}($ voxel $\mid$ component) $\beta$ can be estimated using different algorithms. Gibbs sampling was proposed in the original author-topic paper (RosenZvi et al. 2010). We also proposed a faster expectation maximization (EM) algorithm that was highly efficient on large amount of data (Yeo et al. 2015). In the present work, we used collapsed variational Bayes (CVB) inference (Ngo et al. 2016), which is less sensitive to choice of hyperparameters compared to the EM algorithm. 


\section{2 (S2) Collapsed Variational Bayes (CVB) Inference}

The CVB algorithm for the latent dirichlet allocation model (Blei et al., 2006) was introduced by Teh et al. (2006). We subsequently extended the CVB algorithm to the authortopic model (Ngo et al. 2016). Here we provide the derivation of the algorithm in detail.

We start by following the standard variational Bayesian inference procedure (Beal, 2003) of constructing the lower bound of the log data likelihood:

$$
\begin{aligned}
& \log p(\boldsymbol{v} \mid \alpha, \eta, \boldsymbol{\tau}) \\
& =\log \sum_{\boldsymbol{C}, \boldsymbol{T}} p(\boldsymbol{v}, \boldsymbol{C}, \boldsymbol{T} \mid \alpha, \eta, \boldsymbol{\tau}) \\
& =\log \sum_{\boldsymbol{C}, \boldsymbol{T}} q(\boldsymbol{C}, \boldsymbol{T}) \frac{p(\boldsymbol{v}, \boldsymbol{C}, \boldsymbol{T} \mid \alpha, \eta, \boldsymbol{\tau})}{q(\boldsymbol{C}, \boldsymbol{T})} \\
& =\log E_{q(\boldsymbol{C}, \boldsymbol{T})}\left(\frac{p(\boldsymbol{v}, \boldsymbol{C}, \boldsymbol{T} \mid \alpha, \eta, \boldsymbol{\tau})}{q(\boldsymbol{C}, \boldsymbol{T})}\right) \\
& \geq E_{q(\boldsymbol{C}, \boldsymbol{T})}(\log p(\boldsymbol{v}, \boldsymbol{C}, \boldsymbol{T} \mid \alpha, \eta, \boldsymbol{\tau}))-E_{q(\boldsymbol{C}, \boldsymbol{T})}(\log q(\boldsymbol{C}, \boldsymbol{T}))
\end{aligned}
$$

where $q(\boldsymbol{C}, \boldsymbol{T})$ can be any probability distribution and the inequality (Eq. (1)) utilizes the Jensen inequality (Beal, 2003). We can indirectly maximize the data likelihood $p(\boldsymbol{v} \mid \alpha, \eta, \boldsymbol{\tau})$ by finding the variational distribution $q(\boldsymbol{C}, \boldsymbol{T})$ that maximizes the lower bound (Eq. (1)). The equality in Eq. (1) occurs when $q(\boldsymbol{C}, \boldsymbol{T})=p(\boldsymbol{C}, \boldsymbol{T} \mid \boldsymbol{v}, \alpha, \eta, \boldsymbol{\tau})$, i.e., when the variational distribution is equal to the true posterior distribution. However, computing the true posterior distribution of the latent variables is intractable because of dependencies among the variables constituting $\boldsymbol{C}$ and $\boldsymbol{T}$. Instead, the posterior of the latent variables $(\boldsymbol{C}, \boldsymbol{T})$ is approximated to be factorizable (Teh et al., 2006):

$$
q(\boldsymbol{C}, \boldsymbol{T})=\prod_{e=1}^{E} \prod_{f=1}^{F_{e}} q\left(C_{e f}, T_{e f}\right),
$$

where $q(\boldsymbol{C}, \boldsymbol{T})$ is a categorical distribution with parameters $\phi$ :

$$
q\left(C_{e f}=c, T_{e f}=t\right)=\phi_{e f c t}
$$

By plugging Eq. (3) into Eq. (1), we get 


$$
\begin{aligned}
& \log p(\boldsymbol{v} \mid \alpha, \eta, \boldsymbol{\tau}) \\
& \geq E_{q(\boldsymbol{C}, \boldsymbol{T})}[\log p(\boldsymbol{v}, \boldsymbol{C}, \boldsymbol{T} \mid \alpha, \eta, \boldsymbol{\tau})]-E_{q(\boldsymbol{C}, \boldsymbol{T})}[\log q(\boldsymbol{C}, \boldsymbol{T})] \\
& =\sum_{c=1}^{K} \sum_{t \in \boldsymbol{\tau}_{e}} \phi_{e f c t}\left(E _ { q ( \boldsymbol { C } _ { - e f } , \boldsymbol { T } _ { - e f } ) } \left(\operatorname { l o g } p \left(\boldsymbol{v}, \boldsymbol{C}_{-e f}, \boldsymbol{T}_{-e f}, C_{e f}=c, T_{e f}=\right.\right.\right. \\
& \qquad t \mid \alpha, \eta, \boldsymbol{\tau})))-\sum_{e=1}^{E} \sum_{f=1}^{F_{e}} \sum_{c=1}^{K} \sum_{t \in \boldsymbol{\tau}_{e}} \phi_{e f c t} \log \phi_{e f c t},
\end{aligned}
$$

where the subscript "-ef" indicates the exclusion of corresponding variables $C_{e f}$ and $T_{e f}$. Maximizing the lower bound (Eq. (4)) by differentiating with respect to $\phi$ and using the constraint that $\sum_{c=1}^{K} \sum_{t \in \tau_{e}} \phi_{e f c t}=1$, we get the update equation

$\phi_{\text {efct }}$

$$
=\frac{\exp \left(E_{q\left(\boldsymbol{C}_{-e f}, \boldsymbol{T}_{-e f}\right)}\left(\log p\left(\boldsymbol{v}, \boldsymbol{C}_{-e f}, \boldsymbol{T}_{-e f}, C_{e f}=c, T_{e f}=t \mid \alpha, \eta, \boldsymbol{\tau}\right)\right)\right)}{\sum_{c^{\prime}} \sum_{t \prime \in \boldsymbol{\tau}_{e}} \exp \left(E_{q\left(\boldsymbol{C}_{-e f}, \boldsymbol{T}_{-e f}\right)}\left(\log p\left(\boldsymbol{v}, \boldsymbol{C}_{-e f}, \boldsymbol{T}_{-e f}, C_{e f}=c^{\prime}, T_{e f}=t^{\prime} \mid \alpha, \eta, \boldsymbol{\tau}\right)\right)\right)}
$$

The CVB algorithm involves iterating Eq. (5) till convergence. The remaining derivations concern the evaluation of Eq. (5). We first apply the conditional independence assumptions of the author-topic model to simplify the joint probability distribution in Eq. (5):

$$
\log p(\boldsymbol{v}, \boldsymbol{C}, \boldsymbol{T} \mid \alpha, \eta, \boldsymbol{\tau})=\log p(\boldsymbol{v} \mid \boldsymbol{C}, \eta)+\log p(\boldsymbol{C} \mid \boldsymbol{T}, \alpha)+\log p(\boldsymbol{T} \mid \boldsymbol{\tau})
$$

By exploiting the properties of Dirichlet-multinomial compound distribution (Teh et al., 2006), the first term on the right hand side of Eq. (6) is given by

$$
\begin{aligned}
& \log p(\boldsymbol{v} \mid \boldsymbol{C}, \eta) \\
& \stackrel{\text { (a) }}{=} \log \prod_{c=1}^{K}\left[\frac{\Gamma(V \eta)}{\Gamma\left(V \eta+N_{. . c .}\right)} \prod_{v=1}^{V} \frac{\Gamma\left(\eta+N_{. . c v}\right)}{\Gamma(\eta)}\right] \\
& \stackrel{(b)}{=} \log \prod_{c=1}^{K}\left[\frac{1}{V \eta(V \eta+1) \ldots\left(V \eta+N_{. . c \cdot}-1\right)} \prod_{v=1}^{V} \eta(\eta+1) \ldots\left(\eta+N_{. \cdot c v}-1\right)\right] \\
& =-\sum_{c=1}^{K} \sum_{m=0}^{N_{. . c^{-}-1}} \log (V \eta+m)+\sum_{c=1}^{K} \sum_{v=1}^{V} \sum_{m=0}^{N_{. \cdot c v^{-1}}} \log (\eta+m) \text {, }
\end{aligned}
$$


where equality (7a) arises from the definition of the Dirichlet-multinomial compound distribution and $\Gamma(\cdot)$ is the Gamma function. $N_{\text {etcv }}$ is the number of activation foci in experiment $e$ generated by task $t$, cognitive component $c$, and located at brain location $v$. The dot '.' indicates that the corresponding variable is summed out. For example, $N_{. . c \text {. }}$ is the number of activation foci generated by component $c$ across all experiments. Equation (7b) arises from the identity $\Gamma(z+1)=z \Gamma(z)$ for $z>0$. Using the same procedure, the second term on the right hand side of Eq. (6) can be written as

$$
\log p(\boldsymbol{C} \mid \boldsymbol{T}, \alpha)=-\sum_{t \in \boldsymbol{\tau}} \sum_{m=0}^{N_{t \cdot .-1}} \log (K \alpha+m)+\sum_{t \in \boldsymbol{\tau}} \sum_{c=1}^{K} \sum_{m=0}^{N_{t c c^{-}-1}} \log (\alpha+m)
$$

Substituting Eq. (7) and Eq. (8) back into Eq. (6), we get

$$
\begin{aligned}
& \log p(\boldsymbol{v}, \boldsymbol{C}, \boldsymbol{T} \mid \alpha, \eta, \boldsymbol{\tau}) \\
& =-\sum_{c=1}^{K} \sum_{m=0}^{N_{. . c}-1} \log (V \eta+m)+\sum_{c=1}^{K} \sum_{v=1}^{V} \sum_{m=0}^{N_{. \cdot c v^{-1}}} \log (\eta+m) \\
& -\sum_{t \in \tau} \sum_{m=0}^{N_{t \cdot \cdot}-1} \log (K \alpha+m)+\sum_{t \in \tau} \sum_{c=1}^{K} \sum_{m=0}^{N_{t c \cdot}-1} \log (\alpha+m) \\
& +\log p(\boldsymbol{T} \mid \boldsymbol{\tau}) \text {. }
\end{aligned}
$$

We are now ready to substitute Eq. (9) back into the update Eq. (5). The last term $(\log p(\boldsymbol{T} \mid \boldsymbol{\tau}))$ of Eq. (9) exists in both the numerator and denominator of Eq. (5) and thus cancels out. The remaining terms in Eq. (9) can be similarly simplified as follows. For example, the first term of Eq. (9) can be written as

$$
-\sum_{c=1}^{K} \sum_{m=0}^{N . . c^{-1}} \log (V \eta+m)=\left[-\sum_{c^{\prime}=1}^{K} \sum_{m=0}^{N_{. c^{\prime} \cdot}^{-e f}-1} \log (V \eta+m)\right]-\log \left(V \eta+N_{. . c^{\cdot}}^{-e f}\right)
$$

Therefore when Eq. (9) is substituted back into Eq. (5), the first term of Eq. (10) would be present in both the numerator and denominator of Eq. (5) and cancel out. Using the similar evaluation for the remaining terms, update Eq. (5) becomes 


$$
\begin{aligned}
& \phi_{e f c t} \propto \exp \left(E _ { q ( c _ { - e f } , \boldsymbol { T } _ { - e f } ) } \left(-\log \left(V \eta+N_{. . c^{\cdot}}^{-e f}\right)+\log \left(\eta+N_{. . c v_{e f}}^{-e f}\right)\right.\right. \\
& \left.\left.-\log \left(K \alpha+N_{\cdot t^{*}}^{-e f}\right)+\log \left(\alpha+N_{\cdot t c^{\cdot}}^{-e f}\right)\right)\right)
\end{aligned}
$$

for $t \in \boldsymbol{\tau}_{e}$ (otherwise $\phi_{e f c t}$ is zero), where the first $\log (\cdot)$ term in Eq. (11) comes from the first term in Eq. (9), the second $\log (\cdot)$ term in Eq. (11) comes from the second term in Eq. (9), and so on.

The $\log (\cdot)$ terms in Eq. (10) can be approximated by a second-order Taylor's series expansion about their means (Teh at al. 2006). Consider the Taylor's series expansion of the $\log (b+x)$ function about a particular constant $a$ :

$$
\log (b+x) \approx \log (b+a)+\frac{(x-a)}{b+a}-\frac{(x-a)^{2}}{2(b+a)^{2}}
$$

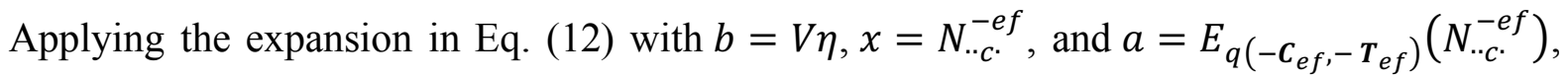
the first term in Eq. (11) can be approximated as

$$
\begin{aligned}
& E_{q\left(\boldsymbol{C}_{-e f}, \boldsymbol{T}_{-e f}\right)}\left(-\log \left(V \eta+N_{. \cdot c^{\cdot}}^{-e f}\right)\right)
\end{aligned}
$$

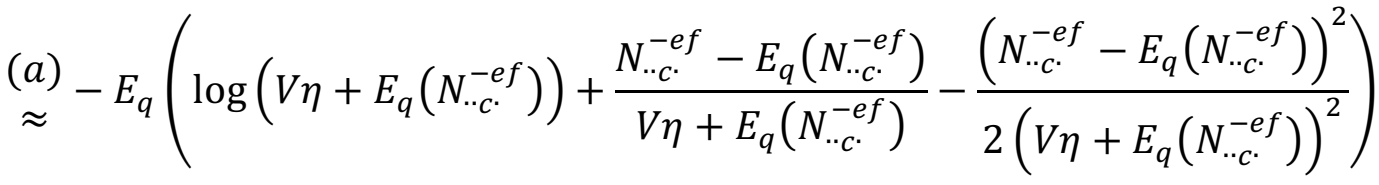

$$
\begin{aligned}
& \stackrel{(b)}{=}-\log \left(V \eta+E_{q}\left(N_{. . c^{\circ}}^{-e f}\right)\right)+\frac{\operatorname{Var}_{q}\left(N_{. . c \cdot}^{-e f}\right)}{2\left(V \eta+E_{q}\left(N_{. . . c}^{-e f}\right)\right)^{2}}
\end{aligned}
$$

where the subscript $\left(\boldsymbol{C}_{-e f}, \boldsymbol{T}_{-e f}\right)$ in $E_{q\left(\boldsymbol{C}_{-e f}, \boldsymbol{T}_{-e f}\right)}(\cdot)$ was omitted in Eq. (13a) to reduce clutter. Eq. (13b) was obtained because the expectation of a constant is itself. Therefore the first term corresponds to $E_{q}\left(\log \left(V \eta+E_{q}\left(N_{. . c^{*}}^{-e f}\right)\right)\right)=\log \left(V \eta+E_{q}\left(N_{. . c^{*}}^{-e f}\right)\right)$. The second term evaluates to zero because $E_{q}\left(E_{q}\left(N_{. \cdot c^{*}}^{-e f}\right)\right)=E_{q}\left(N_{* c^{*} \cdot}^{-e f}\right)$.

Applying the same approximation for all $\log (\cdot)$ terms in Eq. (11) and rearranging, the update equation for $\phi$ becomes 


$$
\begin{aligned}
& \phi_{e f c t} \propto \frac{\left(\eta+E_{q}\left(N_{\cdot \cdot c v_{e f}^{-e f}}^{-e f}\right)\right)\left(\alpha+E_{q}\left(N_{\cdot t c^{\cdot}}^{-e f}\right)\right)}{\left(V \eta+E_{q}\left(N_{\cdot . c^{*}}^{-e f}\right)\right)\left(K \alpha+E_{q}\left(N_{\cdot t^{*}}^{-e f}\right)\right)} \\
& \times \exp \left[\frac{\operatorname{Var}_{q}\left(N_{. \cdot c^{\circ}}^{-e f}\right)}{2\left(V \eta+E_{q}\left(N_{. \cdot c^{\circ}}^{-e f}\right)\right)^{2}}-\frac{\operatorname{Var}_{q}\left(N_{.^{\prime} c v_{e f}}^{-e f}\right)}{2\left(\eta+E_{q}\left(N_{. \cdot c v_{e f}}^{-e f}\right)\right)^{2}}\right] \\
& \times \exp \left[\frac{\operatorname{Var}_{q}\left(N_{\cdot t^{*} \cdot}^{-e f}\right)}{2\left(K \alpha+E_{q}\left(N_{\cdot t^{*}}^{-e f}\right)\right)^{2}}-\frac{\operatorname{Var}_{q}\left(N_{\cdot t c^{\cdot}}^{-e f}\right)}{2\left(\alpha+E_{q}\left(N_{\cdot t c^{*}}^{-e f}\right)\right)^{2}}\right]
\end{aligned}
$$

The mean and variance of the counts in Eq. (14) can be evaluated using the current estimate of $\phi$. For example, $N_{. . c^{*}}^{-e f}$ can be thought of as the number of heads obtained from tossing a coin independently for each focus of each experiment in the entire dataset (excluding the $f$-th focus of the $e$-th experiment), where the probability of getting a head for the $f^{\prime}$-th activated voxel of the $e^{\prime}$-th experiment is equal to $\sum_{t \in \tau_{e}} \phi_{e \prime f \prime c t}$. Thus, the expectation and variance of $N_{. . c}^{-e f}$ is given by

$$
\begin{aligned}
& E_{q}\left(N_{. . c^{\cdot}}^{-e f}\right)=\sum_{e^{\prime} \neq e, f^{\prime} \neq f} \sum_{t \in \tau_{e \prime}} \phi_{e^{\prime} f \prime^{\prime} c t}, \\
& \operatorname{Var}_{q}\left(N_{. . c^{\cdot}}^{-e f}\right)=\sum_{e^{\prime} \neq e, f \prime \neq f}\left(\sum_{t \in \tau_{e \prime}} \phi_{e f^{\prime} f^{\prime c t}}\right)\left(1-\sum_{t \in \boldsymbol{\tau}_{e^{\prime}}} \phi_{e^{\prime} f^{\prime} c t}\right) .
\end{aligned}
$$

By using the same argument for the remaining terms of Eq. (14), we can evaluate the update equation for $\phi_{\text {efct }}$ given the current estimate of $\phi$.

To summarize, the CVB algorithm proceeds by iterating Eq. (14) until convergence. Notice that under CVB inference, the posterior $\phi$ is estimated without using the point estimates of the model parameters $\theta$ and $\beta$ (unlike the EM algorithm; see Supplemental Method S3). Given the final estimate of posterior distribution $\phi$, the parameters $\theta$ and $\beta$ can be estimated by the posterior means (Teh et al. 2006):

$$
\theta_{t c} \propto \alpha+\sum_{e=1}^{E} \sum_{f=1}^{F_{e}} \phi_{e f c t}
$$




$$
\beta_{c v} \propto \eta+\sum_{e=1}^{E} \sum_{f=1}^{F_{e}} \sum_{t \in \boldsymbol{\tau}_{e}} \phi_{e f c t} \mathbb{\mathbb { }}\left(v_{e f}=v\right),
$$

where $\mathbb{I}\left(v_{e f}=v\right)$ equals to one if the activation focus $v_{e f}$ corresponds to location $v$ in MNI152 space, and zero otherwise.

\section{3 (S3) Theoretical differences between CVB with Standard Variational Bayes (SVB) and EM algorithm}

The CVB algorithm is theoretically better than standard variational Bayes (SVB) inference (Teh et al., 2006). As explained in the previous supplemental, CVB algorithm constructs a lower bound to the data log likelihood with respect to the latent variables $(\boldsymbol{C}, \boldsymbol{T})$. On the other hand, the SVB algorithm constructs a lower bound with respect to both latent variables $(\boldsymbol{C}, \boldsymbol{T})$ and model parameters $(\theta, \beta)$. Consequently, CVB provides a tighter lower bound to the data log likelihood:

$$
\begin{aligned}
& \log p(\boldsymbol{v} \mid \alpha, \eta, \boldsymbol{\tau}) \\
& \geq E_{q(\boldsymbol{C}, \boldsymbol{T})}(\log p(\boldsymbol{v}, \boldsymbol{C}, \boldsymbol{T} \mid \alpha, \eta, \boldsymbol{\tau}))-E_{q(\boldsymbol{C}, \boldsymbol{T})}(\log q(\boldsymbol{C}, \boldsymbol{T})) \\
& \geq E_{q(\boldsymbol{C}, \boldsymbol{T}) q(\theta, \beta)}(\log p(\boldsymbol{v}, \boldsymbol{C}, \boldsymbol{T}, \theta, \beta \mid \alpha, \eta, \boldsymbol{\tau}))-E_{q(\theta, \beta)}(\log q(\theta, \beta)) \\
& \quad-E_{q(\boldsymbol{C}, \boldsymbol{T})}(\log q(\boldsymbol{C}, \boldsymbol{T}))
\end{aligned}
$$

where the inequality (Eq. (18)) is the same as CVB Eq. (1) and the second inequality (Eq. (19)) corresponds to SVB.

One can also draw parallels between the CVB (Supplemental Method S2) and EM (Yeo et al., 2015) algorithms for the author-topic model. Both algorithms iterate between estimating the posterior distribution of the latent variables $(\boldsymbol{C}, \boldsymbol{T})$ and using the posterior distribution to update the model parameters estimates $(\theta, \beta)$. However, the EM algorithm uses point estimates of the model parameters to update the posterior distribution of $(\boldsymbol{C}, \boldsymbol{T})$. In contrast, the CVB algorithm avoids doing so (Eq. (14)) and might therefore produce better estimates of the parameters (Ngo et al. 2016).

In practice, we find the CVB algorithm to be less sensitive than the EM algorithm to the initialization of the hyperparameters $\alpha$ and $\eta$. This is not an issue for a big dataset (e.g., BrainMap; Yeo et al., 2015) because the data will overwhelm the priors. However, this issue is important for small datasets like those utilized in this work. For the CVB algorithm, the 
hyperparameters $\alpha$ and $\eta$ were set to 100 and 0.01 respectively across all of our experiments. Perturbing $\alpha$ and $\eta$ by two orders of magnitude did not significantly change the model parameters estimated by CVB algorithm, suggesting its robustness. This was not the case for the EM algorithm.

\section{4 (S4) Estimating Number of Components using Bayesian Information Criterion (BIC)}

Bayesian Information Criterion (BIC) is commonly used for model selection in machine learning (Schwarz, 1978). BIC favors models that best fit the data, while also penalizing models with more parameters. The BIC for the author-topic model is given by:

$$
\begin{aligned}
B I C & =\log p(\boldsymbol{v} \mid \theta, \beta, \boldsymbol{\tau})-\frac{1}{2}\left(k_{\theta}+k_{\beta}\right) \log |\boldsymbol{v}| \\
& =\sum_{e=1}^{E} \log \frac{1}{\left|\boldsymbol{\tau}_{e}\right|} \sum_{f=1}^{F_{e}} \sum_{c=1}^{K} \sum_{t \in \boldsymbol{\tau}_{e}} \beta_{c v_{e f}} \theta_{t c}-\frac{1}{2}\left(k_{\theta}+k_{\beta}\right) \log \sum_{e=1}^{E}\left|\boldsymbol{v}_{e}\right|
\end{aligned}
$$

where the first term is the $\log$ likelihood of the activation foci $v$ given the model parameters estimates $\theta$ and $\beta$, and the second term is the penalty based on the number of model parameters. $\left|\boldsymbol{v}_{\boldsymbol{e}}\right|$ and $\left|\boldsymbol{\tau}_{\boldsymbol{e}}\right|$ are the number of foci and tasks employed in the $e$-th experiment. $k_{\theta}$ and $k_{\beta}$ are the number of free model parameters. $k_{\theta}$ is the number of free parameters in the $M \times K$ matrix $\theta$, which is equal to $M \times(K-1)$ since each row sums to one. $k_{\beta}$ is the approximated number of independent elements in the $K \times V$ matrix $\beta$. Each row of $\beta$ can be interpreted as a spatially smoothed brain image (see Supplemental Method S5). Therefore we approximated the number of independent elements in each row of $\beta$ by the number of resolution elements (resels) in the corresponding brain image (Worsley et al. 1992) using AFNI (Cox 1996).

Models with a higher number of components $K$ fit the data better, resulting in a higher data log likelihood (first term of Eq. (20)). On the other hand, a higher $K$ also increases the number of free parameters $\left(k_{\theta}+k_{\beta}\right)$, which results in a higher penalty (second term of Eq. (20)). A higher BIC values indicates a better model. 


\section{5 (S5) Implementation Details}

The model's hyperparameters were set to be $\alpha=100$ and $\eta=0.01$ across all experiments. Perturbing $\alpha$ and $\eta$ by two orders of magnitude did not significantly change the results. The posterior distribution $\phi$ was randomly initialized. The CVB algorithm then updated the posterior distribution $\phi$ (Eq. (14)) until convergence. Given the estimate of $\phi$, CVB algorithm then computed the model parameters $\theta$ and $\beta$ (Eq. (16) and Eq. (17)). For a given number of components $K$, the procedure was repeated with 1000 random initializations resulting in 1000 estimates. The estimate resulting in the maximum lower bound of the data $\log$ likelihood (Eq. (1)) was taken as the final estimate.

We repeated the procedure with for different number of cognitive components $K$. BIC was computed for each value of $K$ (Supplemental Method S4). Higher BIC implied better model parameters estimates. The model parameters with the highest BIC were presented in the Results and Discussion sections.

\section{6 (S6) Approximation of $\operatorname{Pr}($ co-activation pattern | task)}

For the co-activation analysis of IFJ, each experiment was treated as its own unique task. To help interpret the co-activation pattern in terms of BrainMap task categories (also known as paradigm classes), we estimated the probability of the $c$-th co-activation pattern being utilized by the $t$-th task posthoc:

$$
\operatorname{Pr}(\text { co-activation } c \mid \text { task } t) \propto \sum_{e=1}^{E} \frac{\theta_{e c} \mathbb{\mathbb { }}\left(t \in \boldsymbol{\tau}_{e}\right)}{\left|\boldsymbol{\tau}_{e}\right|}
$$

where $\theta_{e c}$ was the estimated probability that the $e$-th experiment would recruit the $c$-th coactivation pattern, $\boldsymbol{\tau}_{e}$ was the set of tasks utilized in the $e$-th experiment, and $\mathbb{1}\left(t \in \boldsymbol{\tau}_{e}\right)$ is an indicator function that was equal to 1 if the $t$-th task was one of the collection of tasks $\boldsymbol{\tau}_{e}$ utilized by the $e$-th experiment and 0 otherwise. Eq. (22) can be interpreted as weighted average of $\theta_{e c}$ across all experiments utilizing task $t$ with the weight being smaller if an experiment utilizes many tasks. For example, if the 3rd experiment utilized "n-back" and "Stroop" tasks, the probability contributed by this experiment to the computation of the probability of "n-back" recruiting co-activation pattern $\mathrm{C} 1(\operatorname{Pr}($ co - activation $C 1$ | "n back")) would be the probability of the experiment recruiting co-activation pattern $\mathrm{C} 1$ (i.e., $\left.\theta_{31}\right)$, divided by the number of tasks, which is two. 


\section{7 (S7) Self-generated thought studies}

\subsubsection{Summary tables of self-generated thought studies}

\section{Autobiographical memory}

Author N

Addis, Moscovitch, Crawley, \& McAndrews (2004) 14

Andreasen et al. (1995) 13

Denkova, Botzung, \& Manning (2006) 12

Denkova, Botzung, Scheiber, \& Manning (2006) 10

Fink et al. (1996) 7

Gilboa, Winocur, Grady, Hevenor, \& Moscovitch (2004) 9

Graham, Lee, Brett, \& Patterson (2003) 24

Greenberg et al. (2005) 11

Levine et al. (2004) 5

Maddock, Garrett, \& Buonocore (2001) 8

Maguire \& Frith (2003b) 12

ibid. $\quad 12$

Maguire \& Mummery (1999) 8

Maguire, Mummery, \& Buchel (2000) 6

Markowitsch et al. (2000) 8

Piefke, Weiss, Zilles, Markowitsch, \& Fink (2003) 20

Rekkas \& Constable (2005) 12

Tsukiura et al. (2003) 9

Vandekerckhove, Markowitsch, Mertens, \& Woermann (2005) 16

Viard et al. (2007) 12

(Reproduced from Spreng et al., 2009)

\section{Navigation}

\section{Author}

Avila et al. (2006)

Ghaem et al. (1997)

Hartley, Maguire, Spiers, \& Burgess (2003) 16

Iaria, Chen, Guariglia, Ptito, \& Petrides (2007) 9

Jordan, Schadow, Wuestenberg, Heinze, \& Jancke (2004) 8

Kumaran \& Maguire (2005) 18

Maguire, Frackowiak, \& Frith (1997) 11

Maguire et al. (1998) 10

Mayes, Montaldi, Spencer, \& Roberts (2004) 9

Mellet et al. (2002) 6

Pine et al. (2002) 20

Rosenbaum, Ziegler, Winocur, Grady, \& Moscovitch (2004) 10

$\begin{array}{ll}\text { Spiers \& Maguire (2006a) } & 20\end{array}$

(Reproduced from Spreng et al., 2009) 


\section{Task Deactivation}

Author

$\mathbf{N}$

Andreasen et al. (1995)

13

Binder et al. (1999)

Christoff, Ream, \& Gabrieli (2004)

30

12

D'Argembeau et al. (2005)

12

14

Fransson (2006)

24

14

14

13

14

19

63

5

6

McKiernan, Kaufman, Kucera-Thompson, \& Binder (2003) 30

Persson, Lustig, Nelson, \& Reuter-Lorenz (2007) $\quad 60$

Raichle et al. (2001) 19

ibid. $\quad 19$

Shulman et al. (1997) 132

Wicker, Ruby, Royet, \& Fonlupt (2003) 42

(Reproduced from Spreng et al., 2009)

\section{Story-based Theory of Mind}

\section{Author}

Aichorn et al. (2009)

Berthoz et al. (2002a)

Ferstl \& von Cramon (2002)

Fletcher et al. (1995)

9

Gallagher et al. (2000)

6

6

Gobbini et al. (2007)

Jenkins \& Mitchell (2010)

Kobayashi et al. (2006)

ibid.

Mitchell (2008b)

Nieminen-von Wendt et al. (2003)

Perner et al. (2006)

Saxe \& Kanwisher (2003)

Saxe \& Powell (2006)

Saxe et al. (2006b)

Saxe \& Wexler (2005)

Spengler et al. (2009)

Vogeley et al. (2001)

Young et al. (2007)

ibid.

(Reproduced from Mar et al., 2011) 
Nonstory-based Theory of Mind

Author N

Bahneman et al. (2010) 25

Baron-Cohen et al. (1999) 12

Bhatt \& Camerer (2005) 16

Blakemore et al. (2003) 10

$\begin{array}{ll}\text { Brune et al. (2008) } & 13\end{array}$

Brunet et al. (2000) 8

Brunet et al. (2003)

Castelli et al. (2000) 6

Ciaramidaro et al. (2007) 12

Decety et al. (2004) 12

Elliott et al. (2006) 14

Fukui et al. (2006) 16

Gallagher \& Frith (2004) 12

Gallagher et al. (2000) 6

Gallagher et al. (2002) 9

German et al. (2004) 16

Gilbert et al. (2007) 16

Grèzes et al. (2004a) 6

Grèzes et al. (2004b) 11

Hooker et al. (2008) 20

Kana et al. (2009) 12

Kircher et al. (2009) 12

Krach et al. (2009) 12

Lombardo et al. (2010) 33

Malhi et al. (2008) 20

Marjoram et al. (2006) 13

Mitchell et al. (2005a) 18

Ochsner et al. (2005) 16

Platek et al. (2004) 5

Rabin et al. (2010) 20

Rilling et al. (2004) 19

Rilling et al. (2008) 20

Russell et al. (2000)

Samson et al. (2008) 17

$\begin{array}{ll}\text { Sommer et al. (2007) } & 16\end{array}$

Spiers \& Maguire (2006) 20

Spreng \& Grady (2010) 16

Sripada et al. (2009) 26

Vanderwal et al. (2008) 17

Vollm et al. (2006) 13

Walter et al. (2004) 13

ibid. $\quad 12$

Wolf et al. (2010) 18

(Reproduced from Mar et al., 2011) 


\section{Narrative Comprehension}

Author $\quad$ N

Crinion \& Price (2005) 18

Crinion et al. (2003) 11

ibid. 6

Dick et al. (2009) 24

Ferstl \& von Cramon (2001) 12

Giraud et al. (2000) 6

Kansaku et al. (2000) 22

ibid. 25

Kuperberg et al. (2006) 15

Lindenberg \& Scheef (2007) 19

Maguire et al. (1999) 13

Miura et al. (2005) 30

Papathanassiou et al. (2000) 8

Perani et al. (1996) 9

Perani et al. (1998) 9

ibid. $\quad 12$

Robertson et al. (2000) 8

Siebörger et al. (2007) 14

Tzourio et al. (1998) 10

ibid.

(Reproduced from Mar et al., 2011)

\section{Moral Cognition}

Author

Avram et al. (2013) 16

Bahnemann et al. (2010) 25

Borg et al. (2006) 24

Borg et al. (2011) 26

Chiong et al. (2013) 16

FeldmannHall et al. (2012) 14

FeldmannHall et al. (2013) $\quad 35$

Harada et al. (2009) 18

Harenski et al. (2008) 28

Harrison et al. (2008) 22

Hayashi et al. (2010) 12

Heekeren et al. (2003) 8

Heekeren et al. (2005) 12

Moll et al. (2001) 10

Moll et al. (2002a)

Parkinson et al. (2012) 38

Prehn et al. (2008) 23

Reniers et al. (2012) 24

Schleim et al. (2010) 40

Sinke et al. (2010) 14

Sommer et al. (2010) 12 
Takahashi et al. (2008) 15

Akitsuki et al. (2009) 26

Basile et al. (2011) 22

Berhotz et al. (2002) 12

Borg et al. (2008) 15

Decety et al. (2011) 22

$\begin{array}{ll}\text { Finger et al. (2006) } & 16\end{array}$

Harenski et al. (2006) $\quad 30$

Harenski et al. (2010) 14

ImmordinoYang et al. (2009) 13

Kedia et al. (2008) 35

Luo et al. (2006) 20

Mercadillo et al. (2011) 24

Michl et al. (2012) 14

Moll et al. (2002b)

Moll et al. (2005) 13

$\begin{array}{ll}\text { Robertson et al. (2007) } & 16\end{array}$

$\begin{array}{ll}\text { Takahashi et al. (2004) } & 19\end{array}$

Wagner et al. (2011) 15

(Reproduced from Mar et al., 2011)

\subsubsection{References of self-generated thought studies}

\section{Autobiographical memory}

Addis DR, Moscovitch M, Crawley AP, McAndrews MP. 2004. Recollective qualities modulate hippocampal activation during autobiographical memory retrieval. Hippocampus. 14:752-762.

Andreasen NC, O'Leary DS, Cizadlo T, Arndt S, Rezai K, Watkins GL. 1995. Remembering the past: Two facets of episodic memory explored with positron emission tomography. American Journal of Psychiatry. 152:1576-1585.

Denkova E, Botzung A, Manning L. 2006. Neural correlates of remembering/knowing famous people: An event-related fMRI study. Neuropsychologia. 44:2783-2791.

Denkova E, Botzung A, Scheiber C, Manning L. 2006. Implicit emotion during recollection of past events: A nonverbal fMRI study. Brain Research. 1078:143-150.

Fink GR, Markowitsch HJ, Reinkemeier M, Bruckbauer T, Kessler J, Heiss WD. 1996. Cerebral representation of one's own past: Neural networks involved in autobiographical memory. Journal of Neuroscience. 16:4275-4282.

Gilboa A, Winocur G, Grady CL, Hevenor SJ, Moscovitch M. 2004. Remembering our past: Functional neuroanatomy of recollection of recent and very remote personal events. Cerebral Cortex. 14:1214-1225.

Graham KS, Lee ACH, Brett M, Patterson K. 2003. The neural basis of autobiographical and semantic memory: New evidence from three PET studies. Cognitive, Affective, Behavioral Neuroscience. 3:234-254.

Greenberg DL, Rice HJ, Cooper JJ, Cabeza R, Rubin DC, LaBar KS. 2005. Co-activation of the amygdala, hippocampus and inferior frontal gyrus during autobiographical memory retrieval. Neuropsychologia. 43:659-674.

Levine B, Turner GR, Tisserand D, Hevenor SJ, Graham SJ, McIntosh AR. 2004. The functional neuroanatomy of episodic and semantic autobiographical remembering: A prospective functional MRI study. Journal of Cognitive Neuroscience. 16:1633-1646. 
Maddock RF, Garrett AS, Buonocore MH. 2001. Remembering familiar people: The posterior cingulate cortex and autobiographical memory retrieval. Neuroscience. 104:667-676.

Maguire EA, Frith CD. 2003. Aging affects the engagement of the hippocampus during autobiographical memory retrieval. Brain. 126:1511-1523.

Maguire EA, Mummery CJ. 1999. Differential modulation of a common memory retrieval network revealed by positron emission tomography. Hippocampus. 9:54-61.

Maguire EA, Mummery CJ, Buchel C. 2000. Patterns of hippocampal-cortical interaction dissociate temporal lobe memory subsystems. Hippocampus. 10:475-482.

Markowitsch HJ, Thiel A, Reinkemeier M, Kessler J, Koyuncu A, Heiss WD. 2000. Right amygdalar and temporofrontal activation during autobiographic, but not during fictitious memory retrieval. Behavioural Neurology. 12:181-190.

Piefke M, Weiss PH, Zilles K, Markowitsch HJ, Fink GR. 2003. Differential remoteness and emotional tone modulate the neural correlates of autobiographical memory. Brain. 126:650-668.

Rekkas PV, Constable RT. 2005. Evidence that autobiographic memory retrieval does not become independent of the hippocampus: An fMRI study contrasting very recent with remote events. Journal of Cognitive Neuroscience. 17:1950-1961.

Tsukiura T, Fujii T, Okuda J, Ohtake H, Kawashima M, Itoh M. 2003. Time-dependent contribution of the hippocampal complex when remembering the past: A PET study. NeuroReport. 13:2319-2323.

Vandekerckhove MMP, Markowitsch HJ, Mertens M, Woermann FG. 2005. Bi-hemispheric engagement in the retrieval of autobiographical episodes. Behavioural Neurology. 16:203-210.

Viard, A., Piolino, P., Desgranges, B., Chetelat, G., Lebreton, K., Landeau, B. 2007. Hippocampal activation for autobiographical memories over the entire lifetime in healthy aged subjects: An fMRI study. Cerebral Cortex. 17:2453-2467.

\section{Navigation}

Avila C, Barros-Loscertales A, Forn C, Mallo R, Parcet MA, Belloch V. 2006. Memory lateralization with 2 functional MR imaging tasks in patients with lesions in the temporal lobe. American Journal of Neuroradiology. 27:498-503.

Ghaem O, Mellet E, Crivello F, Tzourio N, Mazoyer B, Berthoz A. 1997. Mental navigation along memorized routes activates the hippocampus, precuneus, and insula. NeuroReport. 8:739-744.

Hartley T, Maguire EA, Spiers HJ, Burgess N. 2003. The well-worn route and the path less traveled: Distinct neural bases of route following and wayfinding in humans. Neuron. 37:877-888.

Iaria G, Chen JK, Guariglia C, Ptito A, Petrides M. 2007. Retrosplenial and hippocampal brain regions in human navigation: Complementary functional contributions to the formation and use of cognitive maps. European Journal of Neuroscience. 25:890-899.

Jordan K, Schadow J, Wuestenberg T, Heinze HJ, Jancke L. 2004. Different cortical activations for subjects using allocentric or egocentric strategies in a virtual navigation task. NeuroReport. 15:135-140.

Kumaran D, Maguire EA. 2005. The human hippocampus: Cognitive maps or relational memory? Journal of Neuroscience. 25:7254-7259.

Maguire EA, Frackowiak RSJ, Frith CD. 1997. Recalling routes around London: Activation of the right hippocampus in taxi drivers. Journal of Neuroscience. 17:7103-7110.

Maguire EA, Burgess N, Donnett JG, Frackowiak RSJ, Frith CD, O'Keefe J. 1998. Knowing where and getting there: A human navigation network. Science. 280:921-924. 
Mayes AR, Montaldi D, Spencer TJ, Roberts N. 2004. Recalling spatial information as a component of recently and remotely acquired episodic or semantic memories: An fMRI study. Neuropsychology. 18:426-441.

Mellet E, Bricogne S, Crivello F, Mazoyer B, Denis M, Tzourio-Mazoyer N. 2002. Neural basis of mental scanning of a topographic representation built from a text. Cerebral Cortex. 12:1322-1330.

Pine DS, Grun J, Maguire EA, Burgess N, Zarahn E, Koda V. 2002. Neurodevelopmental aspects of spatial navigation: A virtual reality fMRI study. Neuroimage. 15:396-406.

Rosenbaum RS, Ziegler M, Winocur G, Grady CL, Moscovitch M. 2004. "I have often walked down this street before": fMRI studies on the hippocampus and other structures during mental navigation of an old environment. Hippocampus. 14:826-835.

Spiers HJ, Maguire EA. 2006. Thoughts, behaviour, and brain dynamics during navigation in the real world. Neuroimage. 31:1826-1840.

\section{Task deactivation}

Andreasen NC, O'Leary DS, Cizadlo T, Arndt S, Rezai K, Watkins GL. 1995. Remembering the past: Two facets of episodic memory explored with positron emission tomography. American Journal of Psychiatry. 152:1576-1585.

Binder JR, Frost JA, Hammeke TA, Bellgowan PSF, Rao SM, Cox RW. 1999. Conceptual processing during the conscious resting state: A functional MRI study. Journal of Cognitive Neuroscience. 11:80-93.

Christoff K, Ream JM, Gabrieli JDE. 2004. Neural basis of spontaneous thought processes. Cortex. 40:623-630.

D'Argembeau A, Collette F, Van der Linden M, Laureys S, Del Fiore G, Degueldre C. 2005. Self-referential reflective activity and its relationship with rest: A PET study. Neuroimage. 25:616-624.

Fransson P. 2006. How default is the default mode of brain function? Further evidence from intrinsic BOLD signal fluctuations. Neuropsychologia. 44:2836-2845.

Gould RL, Brown RG, Owen AM, Bullmore ET, Howard RJ. 2006. Task-induced deactivations during successful paired associates learning: An effect of age but not Alzheimer's disease. Neuroimage. 31:818-831.

Greicius MD, Srivastava G, Reiss AL, Menon V. 2004. Default-mode network activity distinguishes Alzheimer's disease from healthy aging: Evidence from functional MRI. Proceedings of the National Academy of Sciences, U.S.A., 101:4637-4642.

Kennedy DP, Redcay E, Courchesne E. 2006. Failing to deactivate: Resting functional abnormalities in autism. Proceedings of the National Academy of Sciences, U.S.A. 103:8275-8280.

Mason MF, Norton MI, Van Horn JD, Wegner DM, Grafton ST, Macrae CN. 2007. Wandering minds: The default network and stimulus-independent thought. Science. 315:393-395.

Mazoyer B, Zago L, Mellet E, Bricogne S, Etard O, Houdé O. 2001. Cortical networks for working memory and executive functions sustain the conscious resting state in man. Brain Research Bulletin. 54:287-298.

McGuire PK, Paulesu E, Frackowiak RSJ, Frith CD. 1996. Brain activity during stimulus independent thought. NeuroReport. 7:2095-2099.

McKiernan KA, Kaufman JN, Kucera-Thompson J, Binder JR. 2003. A parametric manipulation of factors affecting task-induced deactivation in functional neuroimaging. Journal of Cognitive Neuroscience. 15:394-408.

Persson J, Lustig C, Nelson JK, Reuter-Lorenz PA. 2007. Age differences in deactivation: A link to cognitive control? Journal of Cognitive Neuroscience. 19:1021-1032. 
Raichle ME, MacLeod AM, Snyder AZ, Powers WJ, Gusnard DA, Shulman GL. 2001. A default mode of brain function. Proceedings of the National Academy of Sciences, U.S.A. 98:676-682.

Shulman GL, Fiez JA, Corbetta M, Buckner RL, Miezin FM, Raichle ME. 1997. Common blood flow changes across visual tasks:II. Decreases in cerebral cortex. Journal of Cognitive Neuroscience. 9:648-663.

Wicker B, Ruby P, Royet JP, Fonlupt P. 2003. A relation between rest and the self in the brain? Brain Research Reviews. 43:224-230.

\section{Story-based Theory of Mind}

Aichorn M, Perner J, Weiss B, Kronbichler M, Staffen W, Ladurner G. 2009. Temoporoparietal junction activity in theory-of-mind tasks: falseness, beliefs, or attention. Journal of Cognitive Neuroscience. 21:1179-92.

Berthoz S, Armony JL, Blair RJR, Dolan RJ. 2002a. An fMRI study of intentional and unintentional (embarrassing) violations of social norms. Brain 125:1696-708.

Ferstl EC, von Cramon DY. 2002. What does the frontomedian cortex contribute to language processing: coherence or theory of mind? NeuroImage 17:1599-612.

Fletcher PC, Happe F, Frith U, Baker SC, Dolan RJ, Frackowiak RS, Frith CD. 1995. Other minds in the brain: a functional imaging study of "theory of mind" in story comprehension. Cognition. 57:109-28.

Gallagher HL, Happé F, Brunswick N, Fletcher PC, Frith U, Frith CD. 2000. Reading the mind in cartoons and stories: an fMRI study of "theory of mind" in verbal and nonverbal tasks. Neuropsychologia 38:11-21.

Gobbini MI, Koralek AC, Bryan RE, Montgomery KJ, Haxby JV. 2007. Two takes on the social brain: a comparison of theory of mind tasks. Journal of Cognitive Neuroscience. 19:1803- 14 .

Jenkins AC, Mitchell JP. 2010. Mentalizing under uncertainty: dissociated neural responses to ambiguous and unambiguous mental state inferences. Cerebral Cortex. 20:404- 10 .

Kobayashi C, Glover GH, Temple E. 2006. Cultural and linguistic influence on neural bases of "Theory of Mind": an fMRI study with Japanese bilinguals. Brain and Language. 98:210- 20.

Mitchell JP. 2008b. Activity in right temporo-parietal junction is not selective for theory-ofmind. Cerebral Cortex. 18:262- 71.

Nieminen-von Wendt T, Metsähonkala L, Kulomäki T, Aalto S, Autti T, Vanhala R, Von Wendt L. 2003. Changes in cerebral blood flow in Asperger syndrome during theory of mind tasks presented by the auditory route. European Child \& Adolescent Psychiatry. 12:178-89.

Perner J, Aichorn M, Kronbichler M, Staffen W, Ladurner G. 2006. Thinking of mental and other representations: the roles of left and right temporoparietal junction. Social Neuroscience. 1:245-58.

Saxe R, Kanwisher N. 2003. People thinking about people: the role of the temporo-parietal junction in "theory of mind." NeuroImage 19:1835-42.

Saxe R, Powell LJ. 2006. It's the thought that counts: specific brain regions for one component of Theory of Mind. Psychol. Sci. 17:692-99.

Saxe R, Schulz LE, Jiang YV. 2006b. Reading minds versus following rules: dissociating theory of mind and executive control in the brain. Social Neuroscience. 1:284-98.

Saxe R, Wexler A. 2005. Making sense of another mind: the role of the right temporoparietal junction. Neuropsychologia 43:1391- 99.

Spengler S, Yves von Cramon D, Brass M. 2009. Control of shared representations relies on key processes involved in mental state attribution. Human Brain Mapping. 30:3704- 18. 
Vogeley K, Bussfeld P, Newen A, Herrmann S, Happé F, Falkai P, Maier W, Shah NJ, Fink GR, Zilles K. 2001. Mind reading: neural mechanisms of theory of mind and selfperspective. Neuroimage. 14:170-81.

Young L, Cushman F, Hauser M, Saxe R. 2007. The neural basis of the interaction between theory of mind and moral judgment. Proceedings of the National Academy of Sciences USA. 104:8235- 40 .

\section{Nonstory-based Theory of Mind}

Bahnemann M, Dziobek I, Prehn K, Wolf I, Heekeren HR. 2010. Sociotropy in the temporoparietal cortex: common versus distinct processes. Social Cognitive and Affective Neuroscience.5: 48- 58.

Baron-Cohen S, Ring HA, Wheelwright S, Bullmore ET, Brammer MJ, Simmons A, Williams SC. 1999. Social intelligence in the normal and autistic brain: an fMRI study. European Journal of Neuroscience. 11:1891-8.

Bhatt M, Camerer CF. 2005. Self-referential thinking and equilibrium as states of mind in games: fMRI evidence. Games and economic Behavior. 52:424-59.

Blakemore SJ, Boyer P, Pachot-Clouard M, Meltzoff A, Segebarth C, Decety J. 2003. The detection of contingency and animacy from simple animations in the human brain. Cerebral Cortex 13:837- 44.

Brüne M, Lissek S, Fuchs N, Witthaus H, Peters S, Nicolas V, Juckel G, Tegenthoff M. 2008. An fMRI study of theory of mind in schizophrenic patients with "passivity" symptoms. Neuropsychologia. 46:1992-2001.

Brunet E, Sarfati Y, Hardy-Baylé MC, Decety J. 2000. A PET investigation of the attribution of intentions with a nonverbal task. Neuroimage. 11:157- 66.

Brunet E, Sarfati Y, Hardy-Baylé MC, Decety J. 2003. Abnormalities of brain function during a nonverbal theory of mind task in schizophrenia. Neuropsychologia. 41:157482.

Castelli F, Happé F, Frith U, Frith CD. 2000. Movement and mind: a functional imaging study of perception and interpretation of complex intentional patterns. Neuroimage. $12: 314-25$.

Ciaramidaro A, Adenzato M, Enrici I, Erk S, Pia L, Bara BG, Walter H. 2007. The intentional network: how the brain reads varieties of intentions. Neuropsychologia. 45:3105-13.

Decety J, Jackson PL, Sommerville JA, Chaminade T, Meltzoff AN. 2004. The neural bases of cooperation and competition: an fMRI investigation. Neuroimage. 23:744-51.

Elliott R, Völlm B, Drury A, McKie S, Richardson P, William Deakin JF. 2006. Co-operation with another player in a financially rewarded guessing game activates regions implicated in theory of mind. Social neuroscience. 1:385-95.

Fukui H, Murai T, Shinozaki J, Aso T, Fukuyama H, Hayashi T, Hanakawa T. 2006. The neural basis of social tactics: An fMRI study. Neuroimage. 32:913-20.

Gallagher HL, Frith CD. 2004. Dissociable neural pathways for the perception and recognition of expressive and instrumental gestures. Neuropsychologia. 42:1725-36.

Gallagher HL, Happé F, Brunswick N, Fletcher PC, Frith U, Frith CD. 2000. Reading the mind in cartoons and stories: an fMRI study of "theory of mind" in verbal and nonverbal tasks. Neuropsychologia. 38:11- 21.

Gallagher HL, Jack AI, Roepstorff A, Frith CD. 2002. Imaging the intentional stance in a competitive game. Neuroimage. 16:814-21.

German TP, Niehaus JL, Roarty MP, Giesbrecht B, Miller MB. 2004. Neural correlates of detecting pretense: automatic engagement of the intentional stance under covert conditions. Journal of Cognitive Neuroscience. 16:1805- 17. 
Gilbert SJ, Williamson IDM, Dumontheil I, Simons JS, Frith CD, Burgess PW. 2007. Distinct regions of medial rostral prefrontal cortex supporting social and nonsocial functions. Social Cogn. Affect. Neurosci. 2: 217- 26.

Grèzes J, Frith CD, Passingham RE. 2004a. Inferring false beliefs from the actions of oneself and others: an fMRI study. Neuroimage 21: 744- 50.

Grèzes J, Frith CD, Passingham RE. 2004b. Brain mechanisms for inferring deceit in the actions of others. J. Neurosci. 24: 5500- 5.

Hooker CI, Verosky SC, Germine LT, Knight RT, D'Esposito M. 2008. Mentalizing about emotion and its relationship to empathy. Social Cognitive and Affective Neuroscience. 3: $204-17$.

Kana RK, Keller TA, Cherkassky VL, Minshew NJ, Just MA. 2009. Atypical frontalposterior synchronization of Theory of Mind regions in autism during mental state attribution. Soc. Neurosci. 4: 135- 52.

Kircher T, Blumel I, Marjoram D, Lataster T, Karabbendam L, et al. 2009. Online mentalising investigated with functional MRI. Neurosci. Lett. 454: 176-81.

Krach S, Blumel I, Marjoram D, Lataster T, Krabbendam L, et al. 2009. Are women better mindreaders? Sex differences in neural correlates of mentalizing detected with functional MRI. BMC Neurosci. 10: 1- 11 .

Lombardo MV, Chakrabarti B, Bullmore ET, Wheelwright SJ, Sadek SA, et al. 2010. Shared neural circuits for mentalizing about the self and others. J. Cogn.Neuro. 22: 1623-35.

Malhi GS, Lagopoulos J, Das P, Moss K, Berk M, Coulston CM. 2008. A functional MRI study of Theory of Mind in euthymic bipolar disorder patients. Bipolar Disord. 10: 94356.

Marjoram D, Job DE, Whalley HC, Gountouna VE, McIntosh AM, et al. 2006. A visual joke fMRI investigation into Theory of Mind and enhanced risk of schizophrenia.

Neuroimage 31: 1850-58.

Mitchell JP, Banaji MR, Macrae CN. 2005a. The link between social cognition and selfreferential thought in the medial prefrontal cortex. Journal of Cognitive Neuroscience. 17: $1306-15$.

Ochsner KN, Beer JS, Robertson ER, Cooper JC, Gabrieli JD, Kihsltrom JF, D'Esposito M. 2005. The neural correlates of direct and reflected self-knowledge. Neuroimage. 28:797814.

Platek SM, Keenan JP, Gallup Jr GG, Mohamed FB. 2004. Where am I? The neurological correlates of self and other. Cognitive Brain Research. 19:114-22.

Rabin JS, Gilboa A, Stuss DT, Mar RA, Rosenbaum RS. 2010. Common and unique neural correlates of autobiographical memory and theory of mind. Journal of Cognitive Neuroscience. 22:1095-111.

Rilling JK, Sanfey AG, Aronson JA, Nystrom LE, Cohen JD. 2004. The neural correlates of theory of mind within interpersonal interactions. Neuroimage. 22:1694- 708.

Rilling JK, Dagenais JE, Goldsmith DR, Glenn AL, Pagnoni G. 2008. Social cognitive neural networks during in-group and out-group interactions. Neuroimage. 41:1447-61.

Russell TA, Rubia K, Bullmore ET, Soni W, Suckling J, Brammer MJ, Simmons A, Williams SC, Sharma T. 2000. Exploring the social brain in schizophrenia: left prefrontal underactivation during mental state attribution. American journal of psychiatry. 157:2040-2.

Samson AC, Zysset S, Huber O. 2008. Cognitive humor processing: different logical mechanisms in nonverbal cartoons - an fMRI study. Social neuroscience. 3:125-40.

Sommer M, Döhnel K, Sodian B, Meinhart J, Thoermer C, Hajak G. 2007. Neural correlates of true and false belief reasoning. Neuroimage. 35:1378-84.

Spiers HJ, Maguire EA. 2006. Spontaneous mentalizing during an interactive real world task: 
an fMRI study. Neuropsychologia. 44:1674- 82 .

Spreng RN, Grady C. 2010. Patterns of brain activity supporting autobiographical memory, prospection and theory-of-mind and their relationship to the default mode network. Journal of Cognitive Neuroscience. 22:1112- 23.

Sripada CS, Angstadt M, Banks S, Nathan PJ, Liberzon I, Phan KL. 2009. Functional neuroimaging of mentalizing during the trust game in social anxiety disorder. NeuroReport. 20:984-89.

Vanderwal T, Hunyadi E, Grupe DW, Connors CM, Schultz RT. 2008. Self, mother and abstract other: an fMRI study of reflective social processing. Neuroimage. 41:1437-46.

Völlm BA, Taylor AN, Richardson P, Corcoran R, Stirling J, McKie S, Deakin JF, Elliott R. 2006. Neuronal correlates of theory of mind and empathy: a functional magnetic resonance imaging study in a nonverbal task. Neuroimage. 29:90-8.

Walter H, Adenzato M, Ciaramidaro A, Enrici I, Pia L, Bara BG. 2004. Understanding intentions in social interaction: the role of the anterior paracingulate cortex. Journal of Cognitive Neuroscience. 16:1854-63.

Wolf I, Dziobek I, Heekeren HR. 2010. Neural correlates of social cognition in natural settings: a model-free analysis approach. Neuroimage. 49:894-904.

\section{Narrative Comprehension}

Crinion JT, Price CJ. 2005. Right anterior superior temporal activation predicts auditory sentence comprehension following aphasic stroke. Brain. 128:2858-71

Crinion JT, Lambon-Ralph MA, Warburton EA, Howard D, Wise RJS. 2003. Temporal lobe regions engaged during normal speech comprehension. Brain. 126:1193-201

Dick AS, Goldin-Meadow S, Hasson U, Skipper JI, Small SL. 2009. Co-speech gestures influence neural activity in brain regions associated with processing semantic information. Human Brain Mapping. 30:3509-26

Ferstl EC, von Cramon DY. 2001. The role of coherence and cohesion in text comprehension: an event-related fMRI study. Cognitive Brain Research. 11:325-40

Giraud A, Truy E, Frackowiak RSJ, Grégoire M, Pujol JF, Collet L. 2000. Differential recruitment of the speech processing system in healthy subjects and rehabilitated cochlear implant patients. Brain. 123:1391-402

Kansaku K, Yamaura A, Kitazawa S. 2000. Sex differences in lateralization revealed in the posterior language areas. Cerebral Cortex. 10:866-72

Kuperberg GR, Lakshmanan BM, Caplan DN, Holcomb PJ. 2006. Making sense of discourse: an fMRI study of causal inferencing across sentences. NeuroImage. 33:34361

Lindenberg R, Scheef L. 2007. Supramodal language comprehension: role of the left temporal lobe for listening and reading. Neuropsychologia. 45:2407-15

Maguire EA, Frith CD, Morris RGM. 1999. The functional neuroanatomy of comprehension and memory: the importance of prior knowledge. Brain. 122:1839-50

Miura N, Watanabe J, Iwata K, Sassa Y, Riera J, Tsuchiya H, Sato S, Horie K, Takahashi M, Kitamura M, Kawashima R. 2005. Cortical activation during reading of ancient versus modern Japanese texts: fMRI study. Neuroimage. 26:426-31.

Papathanassiou D, Etard O, Mellet E, Zago L, Mazoyer B, Tzourio-Mazoyer N. 2000. A common language network for comprehension and production: a contribution to the definition of language epicenters with PET. NeuroImage. 11:347-57

Perani D, Dehaene S, Grassi F, Cohen L, Cappa SF, Dupoux E, Fazio F, Mehler J. 1996. Brain processing of native and foreign languages. NeuroReport-International Journal for Rapid Communications of Research in Neuroscience. 7:2439-44.

Perani D, Paulesu E, Galles NS, Dupoux E, Dehaene S, Bettinardi V, Cappa SF, Fazio F, 
Mehler J. 1998. The bilingual brain. Proficiency and age of acquisition of the second language. Brain: a journal of neurology. 121:1841-52

Robertson DA, Gernsbacher MA, Guidotti SJ, Robertson RR, Irwin W, Mock BJ, Campana ME. 2000. Functional neuroanatomy of the cognitive process of mapping during discourse comprehension. Psychological science. 11:255-60.

Siebörger FT, Ferstl EC, von Cramon DY. 2007. Making sense of nonsense: an fMRI study of task-induced inference processes during discourse comprehension. Brain Research. 1166:77-91

Tzourio N, Crivello F, Mellet E, Nkanga-Ngila B, Mazoyer B. 1998. Functional anatomy of dominance for speech comprehension in left handers versus right handers. NeuroImage. $8: 1-16$

Xu J, Kemeny S, Park G, Frattali C, Braun A. 2005. Language in context: emergent features of word, sentence, and narrative comprehension. NeuroImage. 25:1002-15

Yarkoni T, Speer NK, Balota DA, McAvoy MP, Zacks JM. 2008a. Pictures of a thousand words: investigating the neural mechanisms of reading with extremely rapid eventrelated fMRI. NeuroImage. 42:973-87

Yarkoni T, Speer NK, Zacks JM. 2008b. Neural substrates of narrative comprehension and memory. NeuroImage. 41:1408-25

\section{Moral Cognition}

Avram M, Gutyrchik E, Bao Y, Pöppel E, Reiser M, Blautzik J. 2012. Neurofunctional correlates of aesthetic and moral judgments. Neuroscience Letters.

Bahnemann M, Dziobek I, Prehn K, Wolf I, Heekeren HR. 2010. Sociotopy in the temporoparietal cortex: common versus distinct processes. Social Cognitive and Affective Neuroscience. 5:48-58.

Borg JS, Hynes C, Van Horn J, Grafton S, Sinnott-Armstrong W. 2006. Consequences, action, and intention as factors in moral judgments: An fMRI investigation. Journal of Cognitive Neuroscience. 18:803-817.

Borg JS, Sinnott-Armstrong W, Calhoun VD, Kiehl KA. 2011. Neural basis of moral verdict and moral deliberation. Social Neuroscience. 6:398-413.

Chiong W, Wilson SM, D'Esposito M, Kayser AS, Grossman SN, Poorzand P, Rankin KP. 2013. The salience network causally influences default mode network activity during moral reasoning. Brain. 136:1929-1941.

FeldmanHall O, Dalgleish T, Thompson R, Evans D, Schweizer S, Mobbs D. 2012. Differential neural circuitry and self-interest in real vs hypothetical moral decisions. Social Cognitive and Affective Neuroscience. 7:743-751.

FeldmanHall O, Mobbs D, Dalgleish T. 2013. Deconstructing the brain's moral network: dissociable functionality between the temporoparietal junction and ventro-medial prefrontal cortex. Social Cognitive and Affective Neuroscience.

Harada T, Itakura S, Xu F, Lee K, Nakashita S, Saito DN, Sadato N. 2009. Neural correlates of the judgment of lying: A functional magnetic resonance imaging study. Neuroscience research. 63:24-34.

Harenski CL, Antonenko O, Shane MS, Kiehl KA. 2008. Gender differences in neural mechanisms underlying moral sensitivity. Social Cognitive and Affective Neuroscience. 3:313-321.

Harrison BJ, Pujol J, López-Solà M, Hernández-Ribas R, Deus J, Ortiz H, Cardoner N. 2008. Consistency and functional specialization in the default mode brain network.

Proceedings of the National Academy of Sciences. 105:9781-9786.

Hayashi A, Abe N, Ueno A, Shigemune Y, Mori E, Tashiro M, Fujii T. 2010. Neural correlates of forgiveness for moral transgressions involving deception. Brain Research. 


\section{2:90-99.}

Heekeren HR, Wartenburger I, Schmidt H, Schwintowski HP, Villringer A. 2003. An fMRI study of simple ethical decision-making. Neuroreport. 14:1215-1219.

Heekeren HR, Wartenburger I, Schmidt H, Prehn K, Schwintowski HP, Villringer A. 2005. Influence of bodily harm on neural correlates of semantic and moral decision-making. Neuroimage. 24:887-897.

Moll J, Eslinger PJ, Oliveira-Souza RD. 2001. Frontopolar and anterior temporal cortex activation in a moral judgment task: preliminary functional MRI results in normal subjects. Arquivos de neuro-psiquiatria. 59:657-664.

Moll J, de Oliveira-Souza R, Bramati IE, Grafman J. 2002. Functional networks in emotional moral and nonmoral social judgments. Neuroimage. 16:696-703.

Parkinson C, Sinnott-Armstrong W, Koralus PE, Mendelovici A, McGeer V, Wheatley T. 2011. Is morality unified? Evidence that distinct neural systems underlie moral judgments of harm, dishonesty, and disgust. Journal of Cognitive Neuroscience. 23:3162-3180.

Prehn K, Wartenburger I, Mériau K, Scheibe C, Goodenough OR, Villringer A, Heekeren HR. 2008. Individual differences in moral judgment competence influence neural correlates of socio-normative judgments. Social Cognitive and Affective Neuroscience. 3:33-46.

Reniers RL, Corcoran R, Völlm BA, Mashru A, Howard R, Liddle PF. 2012. Moral decisionmaking, ToM, empathy and the default mode network. Biological psychology. 90:202210.

Schleim S, Spranger TM, Erk S, Walter H. 2011. From moral to legal judgment: the influence of normative context in lawyers and other academics. Social Cognitive and Affective Neuroscience. 6:48-57.

Sinke CBA, Sorger B, Goebel R, de Gelder B. 2010. Tease or threat? Judging social interactions from bodily expressions. Neuroimage. 49:1717-1727.

Sommer M, Rothmayr C, Döhnel K, Meinhardt J, Schwerdtner J, Sodian B, Hajak G. 2010. How should I decide? The neural correlates of everyday moral reasoning. Neuropsychologia. 48:2018-2026.

Takahashi H, Kato M, Matsuura M, Koeda M, Yahata N, Suhara T, Okubo Y. 2008. Neural correlates of human virtue judgment. Cerebral Cortex. 18:1886-1891.

Akitsuki Y, Decety J. 2009. Social context and perceived agency affects empathy for pain: an event-related fMRI investigation. Neuroimage. 47:722-734.

Basile B, Mancini F, Macaluso E, Caltagirone C, Frackowiak RS, Bozzali M. 2011. Deontological and altruistic guilt: Evidence for distinct neurobiological substrates. Human brain mapping. 32:229-239.

Berthoz S, Armony JL, Blair RJR, Dolan RJ. 2002. An fMRI study of intentional and unintentional (embarrassing) violations of social norms. Brain. 125:1696-1708.

Borg JS, Lieberman D, Kiehl KA. 2008. Infection, incest, and iniquity: Investigating the neural correlates of disgust and morality. Journal of Cognitive Neuroscience. 20:15291546.

Decety J, Porges EC. 2011. Imagining being the agent of actions that carry different moral consequences: an fMRI study. Neuropsychologia. 49:2994-3001.

Finger EC, Marsh AA, Kamel N, Mitchell DG, Blair JR. 2006. Caught in the act: the impact of audience on the neural response to morally and socially inappropriate behavior. Neuroimage. 33:414-421.

Harenski CL, Hamann S. 2006. Neural correlates of regulating negative emotions related to moral violations. Neuroimage. 30:313-324.

Harenski CL, Antonenko O, Shane MS, Kiehl KA. 2010. A functional imaging investigation of moral deliberation and moral intuition. Neuroimage. 49:2707-2716. 
Immordino-Yang MH, McColl A, Damasio H, Damasio A. 2009. Neural correlates of admiration and compassion. Proceedings of the National Academy of Sciences. 106:8021-8026.

Kédia G, Berthoz S, Wessa M, Hilton D, Martinot JL. 2008. An agent harms a victim: a functional magnetic resonance imaging study on specific moral emotions. Journal of Cognitive Neuroscience. 20:1788-1798.

Luo Q, Nakic M, Wheatley T, Richell R, Martin A, Blair RJR. 2006. The neural basis of implicit moral attitude — an IAT study using event-related fMRI. Neuroimage. 30:14491457.

Mercadillo RE, Díaz JL, Pasaye EH, Barrios FA. 2011. Perception of suffering and compassion experience: brain gender disparities. Brain and Cognition. 76:5-14.

Michl P, Meindl T, Meister F, Born C, Engel RR, Reiser M, Hennig-Fast K. 2012.

Neurobiological underpinnings of shame and guilt: A pilot fMRI study. Social Cognitive and Affective Neuroscience.

Moll J, de Oliveira-Souza R, Eslinger PJ, Bramati IE, Mourão-Miranda J, Andreiuolo PA, Pessoa L. 2002. The neural correlates of moral sensitivity: a functional magnetic resonance imaging investigation of basic and moral emotions. The Journal of Neuroscience. 22:2730-2736.

Moll J, de Oliveira-Souza R, Moll FT, Ignácio FA, Bramati IE, Caparelli-Dáquer EM, Eslinger PJ. 2005. The moral affiliations of disgust: A functional MRI study. Cognitive and behavioral neurology. 18:68-78.

Robertson D, Snarey J, Ousley O, Harenski K, Bowman FD, Gilkey R, Kilts C. 2007. The neural processing of moral sensitivity to issues of justice and care. Neuropsychologia. 45:755-766.

Takahashi H, Yahata N, Koeda M, Matsuda T, Asai K, Okubo Y. 2004. Brain activation associated with evaluative processes of guilt and embarrassment: an fMRI study. Neuroimage. 23:967-974.

Wagner U, N'Diaye K, Ethofer T, Vuilleumier P. 2011. Guilt-specific processing in the prefrontal cortex. Cerebral Cortex. 21:2461-2470. 


\section{8 (S8) Inferior Frontal Junction (IFJ) studies}

\subsubsection{Summary table of IFJ studies}

Inferior Frontal Junction

\begin{tabular}{|c|c|c|c|}
\hline Author & $\mathbf{N}$ & Author & $\mathbf{N}$ \\
\hline Buckner et al. (2000) & 12 & Devlin et al. (2003) & 12 \\
\hline Buckner et al. (1998) & 26 & ibid. & 12 \\
\hline Bush et al. (1998) & 9 & Kawashima et al. (1996) & 9 \\
\hline Garavan et al. (2000) & 5 & Baker et al. (1997) & 10 \\
\hline Kapur et al. (1996) & 12 & Braver et al. (1997) & 8 \\
\hline Schmidt et al. (2002) & 12 & Curtis et al. (1998) & 5 \\
\hline Sperling et al. (2001) & 8 & Gemar et al. (1996) & 11 \\
\hline Zatorre et al. (1998) & 10 & Gruber et al. (2003) & 13 \\
\hline ibid. & 10 & Tatsumi et al. (1999) & 6 \\
\hline Dehaene et al. (2001) & 37 & Chen et al. (2002) & 9 \\
\hline ibid. & 37 & Démonet et al. (1994) & 9 \\
\hline Bunge et al. (2001) & 16 & Jonides et al. (1997) & 9 \\
\hline Calvert et al. (2003) & 8 & ibid. & 19 \\
\hline ibid. & 8 & Kuo et al. (2001) & 7 \\
\hline ibid. & 8 & Martinkauppi et al. (2000) & 10 \\
\hline Decety et al. (1994) & 6 & Xiang et al. (2003) & 6 \\
\hline ibid. & 6 & Milham et al. (2001) & 16 \\
\hline Fincham et al. (2002) & 8 & Griffiths et al. (1998) & 3 \\
\hline Köhler et al. (1998) & 12 & Konishi et al. (2002) & 16 \\
\hline Papathanassiou et al. (2000) & 8 & Monchi et al. (2001) & 11 \\
\hline ibid. & 8 & ibid. & 11 \\
\hline Petit et al. (1999) & 10 & Thompson-Schill et al. (1999) & 5 \\
\hline Xiong et al. (2000) & 20 & ibid. & 5 \\
\hline Zago et al. (2001) & 6 & ibid. & 5 \\
\hline ibid. & 6 & Landau et al. (2004) & 10 \\
\hline ibid. & 6 & Noppeney et al. (2004) & 15 \\
\hline Dassonville et al. (2001) & 8 & Wheaton et al. (2004) & 12 \\
\hline ibid. & 8 & Jackson et al. (2004) & 12 \\
\hline ibid. & 8 & LaBar et al. (1999) & 11 \\
\hline Mazard et al. (2002) & 6 & Milham et al. (2005) & 18 \\
\hline Mechelli et al. (2000) & 6 & Poeppel et al. (2004) & 10 \\
\hline ibid. & 6 & ibid. & 10 \\
\hline Nunn et al. (2002) & 27 & ibid. & 10 \\
\hline Rossell et al. (2002) & 6 & Astafiev et al. (2003) & 15 \\
\hline ibid. & 6 & Beauchamp et al. (2001) & 9 \\
\hline Rossell et al. (2001) & 12 & Berman et al. (1995) & 40 \\
\hline Shergill et al. (2001) & 8 & Cohe et al. (2003) & 9 \\
\hline Ruff et al. (2001) & 12 & Connolly et al. (2000) & 7 \\
\hline Abel et al. (2003) & 8 & Konishi et al. (2003) & 16 \\
\hline Mead et al. (2002) & 18 & Kuo et al. (2003) & 8 \\
\hline Chee et al. (1999) & 7 & Nagahama et al. (2001) & 6 \\
\hline ibid. & 8 & Nakamura et al. (2000) & 10 \\
\hline ibid. & 8 & & \\
\hline
\end{tabular}


Inferior Frontal Junction

\begin{tabular}{|c|c|c|c|}
\hline Author & $\mathbf{N}$ & Author & $\mathbf{N}$ \\
\hline $\mathrm{Xu}$ et al. (2001) & 6 & Vitali et al. (2005) & 10 \\
\hline ibid. & 6 & ibid. & 10 \\
\hline ibid. & 6 & Manoach et al. (2000) & 9 \\
\hline ibid. & 6 & Poldrack et al. (2005) & 13 \\
\hline ibid. & 6 & Pierce et al. (2004) & 9 \\
\hline ibid. & 6 & Tremblay et al. (2006) & 12 \\
\hline ibid. & 6 & Van et al. (2005) & 10 \\
\hline ibid. & 6 & ibid. & 10 \\
\hline Booth et al. (2002) & 13 & Weiss et al. (2004) & 9 \\
\hline ibid. & 13 & Zhang et al. (2005) & 22 \\
\hline Chochon et al. (1999) & 8 & Gurd et al. (2002) & 11 \\
\hline Delazer et al. (2003) & 13 & Meisenzahl et al. (2006) & 12 \\
\hline Kuo et al. (2004) & 10 & Wang et al. (2004) & 12 \\
\hline Pesenti et al. (2000) & 8 & Voets et al. (2005) & 12 \\
\hline Kosslyn et al. (1998) & 12 & Dichter et al. (2007) & 17 \\
\hline Wraga et al. (2003) & 16 & Kraut et al. (2006) & 18 \\
\hline Norris et al. (2002) & 7 & Ravnkilde et al. (2002) & 46 \\
\hline ibid. & 7 & Uher et al. (2006) & 8 \\
\hline Omori et al. (1999) & 6 & Jobard et al. (2007) & 10 \\
\hline Brass et al. (2002) & 11 & Wong et al. (2007) & 17 \\
\hline ibid. & 11 & Frankenstein et al. (2001) & 12 \\
\hline ibid. & 11 & ibid. & 12 \\
\hline ibid. & 11 & Garavan et al. (2000) & 11 \\
\hline Brass et al. (2004) & 14 & Bohland et al. (2006) & 13 \\
\hline Giesbrecht et al. (2003) & 10 & Dupont et al. (2002) & 10 \\
\hline Kim et al. (1999) & 10 & Addis et al. (2007) & 14 \\
\hline Sylvester et al. (2003) & 14 & Koshino et al. (2007) & 11 \\
\hline ibid. & 14 & Nakao et al. (2005) & 14 \\
\hline Zysset et al. (2001) & 9 & Fitzgerald et al. (2008) & 13 \\
\hline ibid. & 9 & Vuilleumier et al. (2001) & 12 \\
\hline Heim et al. (2002) & 12 & Maril et al. (2003) & 17 \\
\hline Cunnington et al. (2006) & 14 & De et al. (2003) & 11 \\
\hline Ino et al. (2004) & 13 & Carreiras et al. (2007) & 36 \\
\hline Hamzei et al. (2003) & 12 & ibid. & 36 \\
\hline Heckers et al. (2004) & 15 & ibid. & 36 \\
\hline Weiss et al. (2003) & 13 & ibid. & 36 \\
\hline Hofer et al. (2003) & 10 & ibid. & 36 \\
\hline ibid. & 10 & ibid. & 36 \\
\hline Yoo et al. (2005) & 10 & Diedrichsen et al. (2006) & 15 \\
\hline Audoin et al. (2005) & 18 & Johnson et al. (2002) & 8 \\
\hline Monks et al. (2004) & 12 & Nunez et al. (2005) & 20 \\
\hline ibid. & 12 & Postle et al. (2006) & 12 \\
\hline Miura et al. (2005) & 30 & Neuner et al. (2007) & 15 \\
\hline Mo et al. (2005) & 8 & Noriuchi et al. (2008) & 13 \\
\hline Poldrack et al. (1999) & 8 & Coan et al. (2006) & 16 \\
\hline Pihlajamäki et al. (2000) & 14 & Sadato et al. (2004) & 9 \\
\hline
\end{tabular}




\section{Inferior Frontal Junction}

Author N

Ernst et al. (2004)

ibid.

Blair et al. (2006)

ibid.

Marsh et al. (2007)

Ranganath et al. (2004)

Bartels et al. (2004)

Grimm et al. (2006)

ibid.

Dolcos et al. (2004)

Iseki et al. (2008)

Meister et al. (2007)

Ogawa et al. (2005)

Aziz et al. (2000)

Carr et al. (2003)

ibid.

ibid.

Iadarola et al. (1998)

Jacobsen et al. (2006)

Kranz et al. (2006)

Leibenluft et al. (2004)

Phillips et al. (2004)

ibid.

ibid.

Wong et al. (2008)

Yassa et al. (2008)

Martin et al. (2007)

Wicker et al. (2003)

Ferrandez et al. (2003)

ibid.

Herpertz et al. (2008)

Jabbi et al. (2007)

Lanius et al. (2004)

Lanius et al. (2005)

Gruber et al. (2010)

Beneventi et al. (2007)

Brambati et al. (2006)

Brown et al. (2007)

Hauk et al. (2008)

Lai et al. (2009)

LoPresti et al. (2008)

Malisza et al. (2005)

ibid.

Sergerie et al. (2009)

Yoo et al. (2007)

David et al. (2006)

Ochsner et al. (2005)

\section{$\mathbf{N}$}

17

17

21

21

18

13

20

29

29

16

16

14

11

6

11

11

11

13

15

10

7

8

8

8

11

18

\section{8}

14

\section{Author}

N

Chikazoe et al. (2009) 22

ibid.

22

ibid.

22

Davis et al. (2009)

27

Kato et al. (2009)

40

Small et al. (2009)

12

ibid.

12

10

6

11

10

14

14

14

14

10

22

22

22

14

16

10

21

21

17

8

27

25

18

15

20

20

24

24

30

14

15

15

12

12

16

18

9

36

24

24

24 


\section{Inferior frontal junction}

\begin{tabular}{llll}
\hline Author & $\mathbf{N}$ & Author & $\mathbf{N}$ \\
Paulesu et al. (2003) & 8 & Marvel et al. (2012) & 18 \\
ibid. & 8 & ibid. & 12 \\
ibid. & 8 & Kadosh et al. (2005) & 12 \\
ibid. & 8 & Clapp et al. (2009) & 38 \\
ibid. & 8 & Mecklinger et al. (2003) & 11 \\
O'sullivan et al. (1995) & 18 & Bodden et al. (2013) & 11 \\
Pompei et al. (2011) & 48 & Montojo et al. (2008) & 32 \\
ibid. & 25 & Das et al. (2012) & 22 \\
Zandbelt et al. (2011) & 22 & Abrahams et al. (2003) & 22 \\
ibid. & 22 & Abutalebi et al. (2007) & 13 \\
Marangolo et al. (2006) & 10 & ibid. & 10 \\
ibid. & 10 & Bitan et al. (2007) & 8 \\
Uchiyama et al. (2006) & 20 & Booth et al. (2003) & 8 \\
Aziz-Zadeh et al. (2006) & 12 & ibid. & 8 \\
ibid. & 12 & Braskie et al. (2009) & 8 \\
Fernández-Corcuera et al. (2013) & 41 & Seo et al. (2012) & 12 \\
Newman et al. (2011) & 15 & Lutz et al. (2013) & 12 \\
Mickley et al. (2009) & 19 & Callan et al. (2010) & 14 \\
Sergerie et al. (2006) & 18 & Carbon et al. (2010) & 16 \\
Gray et al. (2003) & 60 & Chee et al. (1999) & 16 \\
Kong et al. (2005) & 16 & ibid. & 15 \\
ibid. & 16 & ibid. & 22 \\
ibid. & 16 & ibid. & 12 \\
ibid. & 16 & Chen et al. (2008) & 30 \\
Huang et al. (2012) & 15 & Parsons et al. (2005) & 16 \\
Gutchess et al. (2012) & 16 & Ragland et al. (2005) & 19 \\
& & &
\end{tabular}

\subsubsection{References of IFJ studies}

Abel KM, Allin MP, Kucharska-Pietura K, Andrew C, Williams S, David AS, Phillips ML. 2003. Ketamine and fMRI BOLD signal: distinguishing between effects mediated by change in blood flow versus change in cognitive state. Human Brain Mapping. 18:13545.

Abrahams S, Goldstein LH, Simmons A, Brammer MJ, Williams SC, Giampietro VP, Andrew CM, Leigh PN. 2003. Functional magnetic resonance imaging of verbal fluency and confrontation naming using compressed image acquisition to permit overt responses. Human brain mapping. 20:29-40.

Abutalebi J, Keim R, Brambati SM, Tettamanti M, Cappa SF, De Bleser R, Perani D. 2007. Late acquisition of literacy in a native language. Human Brain Mapping. 28:19-33.

Addis DR, Wong AT, Schacter DL. 2007. Remembering the past and imagining the future: common and distinct neural substrates during event construction and elaboration. Neuropsychologia. 45:1363-77.

Astafiev SV, Shulman GL, Stanley CM, Snyder AZ, Van Essen DC, Corbetta M. 2003. Functional organization of human intraparietal and frontal cortex for attending, looking, and pointing. Journal of Neuroscience. 23:4689-99.

Audoin B, Van Au Duong M, Ranjeva JP, Ibarrola D, Malikova I, Confort-Gouny S, Soulier E, Viout P, Ali-Chérif A, Pelletier J, Cozzone PJ. 2005. Magnetic resonance study of the 
influence of tissue damage and cortical reorganization on PASAT performance at the earliest stage of multiple sclerosis. Human Brain Mapping. 24:216-28.

Aziz Q, Thompson DG, Ng VW, Hamdy S, Sarkar S, Brammer MJ, Bullmore ET, Hobson A, Tracey I, Gregory L, Simmons A. 2000. Cortical processing of human somatic and visceral sensation. Journal of Neuroscience. 20:2657-63.

Aziz-Zadeh L, Wilson SM, Rizzolatti G, Iacoboni M. 2006. Congruent embodied representations for visually presented actions and linguistic phrases describing actions. Current Biology. 16:1818-23.

Baker SC, Frith CD, Dolan RJ. 1997. The interaction between mood and cognitive function studied with PET. Psychological Medicine. 27:565-78.

Bartels A, Zeki S. 2004. The neural correlates of maternal and romantic love. Neuroimage. 21:1155-66.

Beauchamp MS, Petit L, Ellmore TM, Ingeholm J, Haxby JV. 2001. A parametric fMRI study of overt and covert shifts of visuospatial attention. Neuroimage. 14:310-21.

Beneventi H, Barndon R, Ersland L, Hugdahl K. 2007. An fMRI study of working memory for schematic facial expressions. Scandinavian Journal of Psychology. 48:81-6.

Berlingeri M, Crepaldi D, Roberti R, Scialfa G, Luzzatti C, Paulesu E. 2008. Nouns and verbs in the brain: Grammatical class and task specific effects as revealed by fMRI. Cognitive Neuropsychology. 25:528-58.

Berman KF, Ostrem JL, Randolph C, Gold J, Goldberg TE, Coppola R, Carson RE, Herscovitch P, Weinberger DR. 1995. Physiological activation of a cortical network during performance of the Wisconsin Card Sorting Test: a positron emission tomography study. Neuropsychologia. 33:1027-46.

Binder JR, Medler DA, Desai R, Conant LL, Liebenthal E. 2005. Some neurophysiological constraints on models of word naming. Neuroimage. 27:677-93.

Bitan T, Burman DD, Chou TL, Lu D, Cone NE, Cao F, Bigio JD, Booth JR. 2007. The interaction between orthographic and phonological information in children: an fMRI study. Human brain mapping. 28:880-91.

Blair K, Marsh AA, Morton J, Vythilingam M, Jones M, Mondillo K, Pine DC, Drevets WC, Blair JR. 2006. Choosing the lesser of two evils, the better of two goods: specifying the roles of ventromedial prefrontal cortex and dorsal anterior cingulate in object choice. Journal of Neuroscience. 26:11379-86.

Bodden ME, Kübler D, Knake S, Menzler K, Heverhagen JT, Sommer J, Kalbe E, Krach S, Dodel R. 2013. Comparing the neural correlates of affective and cognitive theory of mind using fMRI: Involvement of the basal ganglia in affective theory of mind. Advances in Cognitive Psychology. 9:32.

Bohland JW, Guenther FH. An fMRI investigation of syllable sequence production. 2006. Neuroimage. 32:821-41.

Booth JR, Burman DD, Meyer JR, Gitelman DR, Parrish TB, Mesulam MM. 2002. Functional anatomy of intra-and cross-modal lexical tasks. Neuroimage. 16:7-22.

Booth JR, Burman DD, Meyer JR, Gitelman DR, Parrish TB, Mesulam MM. 2003. Relation between brain activation and lexical performance. Human Brain Mapping. 19:155-69.

Brambati SM, Termine C, Ruffino M, Danna M, Lanzi G, Stella G, Cappa SF, Perani D. 2006. Neuropsychological deficits and neural dysfunction in familial dyslexia. Brain Research. 1113:174-85.

Braskie MN, Small GW, Bookheimer SY. 2009. Entorhinal cortex structure and functional MRI response during an associative verbal memory task. Human Brain Mapping. 30:3981-92.

Brass M, Cramon DY. 2004. Decomposing components of task preparation with functional magnetic resonance imaging. Journal of cognitive neuroscience. 16:609-20. 
Brass M, von Cramon DY. 2002. The role of the frontal cortex in task preparation. Cerebral Cortex. 12:908-14.

Braver TS, Cohen JD, Nystrom LE, Jonides J, Smith EE, Noll DC. 1997. A parametric study of prefrontal cortex involvement in human working memory. Neuroimage. 5:49-62.

Brown S, Martinez MJ. 2007. Activation of premotor vocal areas during musical discrimination. Brain and cognition. 63:59-69.

Buckner RL, Koutstaal W, Schacter DL, Rosen BR. 2000. Functional MRI evidence for a role of frontal and inferior temporal cortex in amodal components of priming. Brain. 123:620-40.

Buckner RL, Koutstaal W, Schacter DL, Wagner AD, Rosen BR. 1998. Functional-anatomic study of episodic retrieval using fMRI: I. Retrieval effort versus retrieval success. Neuroimage. 7:151-62.

Bunge SA, Ochsner KN, Desmond JE, Glover GH, Gabrieli JD. 2001. Prefrontal regions involved in keeping information in and out of mind. Brain. 124:2074-86.

Bush G, Whalen PJ, Rosen BR, Jenike MA, McInerney SC, Rauch SL. 1998. The counting Stroop: an interference task specialized for functional neuroimaging - validation study with functional MRI. Human brain mapping. 6:270-82.

Callan DE, Schweighofer N. 2010. Neural correlates of the spacing effect in explicit verbal semantic encoding support the deficient-processing theory. Human Brain Mapping. 31:645-59.

Calvert GA, Campbell R. 2003. Reading speech from still and moving faces: the neural substrates of visible speech. Journal of cognitive neuroscience. 15:57-70.

Carbon M, Reetz K, Ghilardi MF, Dhawan V, Eidelberg D. 2010. Early Parkinson's disease: longitudinal changes in brain activity during sequence learning. Neurobiology of Disease. 37:455-60.

Carr L, Iacoboni M, Dubeau MC, Mazziotta JC, Lenzi GL. 2003. Neural mechanisms of empathy in humans: a relay from neural systems for imitation to limbic areas. Proceedings of the national Academy of Sciences. 100:5497-502.

Carreiras M, Mechelli A, Estévez A, Price CJ. 2007. Brain activation for lexical decision and reading aloud: two sides of the same coin?. Journal of cognitive neuroscience. 19:43344.

Chee MW, O'Craven KM, Bergida R, Rosen BR, Savoy RL. 1999. Auditory and visual word processing studied with fMRI. Human brain mapping. 7:15-28.

Chee MW, Tan EW, Thiel T. 1999. Mandarin and English single word processing studied with functional magnetic resonance imaging. Journal of Neuroscience. 19:3050-6.

Chee MW, Venkatraman V, Westphal C, Siong SC. 2003. Comparison of block and eventrelated fMRI designs in evaluating the word-frequency effect. Human Brain Mapping. 18:186-93.

Chen JL, Penhune VB, Zatorre RJ. 2008. Listening to musical rhythms recruits motor regions of the brain. Cerebral Cortex. 18:2844-54.

Chen Y, Fu S, Iversen SD, Smith SM, Matthews PM. 2002. Testing for dual brain processing routes in reading: a direct contrast of Chinese character and pinyin reading using fMRI. Journal of Cognitive Neuroscience. 14:1088-98.

Chikazoe J, Jimura K, Hirose S, Yamashita KI, Miyashita Y, Konishi S. 2009. Preparation to inhibit a response complements response inhibition during performance of a stop-signal task. Journal of Neuroscience. 29:15870-7.

Chochon F, Cohen L, Moortele PV, Dehaene S. 1999. Differential contributions of the left and right inferior parietal lobules to number processing. Journal of Cognitive Neuroscience. 11:617-30.

Clapp WC, Rubens MT, Gazzaley A. 2009. Mechanisms of working memory disruption by 
external interference. Cerebral Cortex. 20:859-72.

Coan JA, Schaefer HS, Davidson RJ. 2006. Lending a hand: Social regulation of the neural response to threat. Psychological Science. 17:1032-9.

Cohen L, Martinaud O, Lemer C, Lehéricy S, Samson Y, Obadia M, Slachevsky A, Dehaene S. 2003. Visual word recognition in the left and right hemispheres: anatomical and functional correlates of peripheral alexias. Cerebral Cortex. 13:1313-33.

Coletta M, Platek S, Mohamed FB, van Steenburgh JJ, Green D, Lowe MR. 2009. Brain activation in restrained and unrestrained eaters: an fMRI study. Journal of abnormal psychology. 118:598.

Connolly JD, Goodale MA, Desouza JF, Menon RS, Vilis T, Medical Research Council Group for Action and Perception). 2000. A comparison of frontoparietal fMRI activation during anti-saccades and anti-pointing. Journal of neurophysiology. 84:1645-55.

Cunnington R, Windischberger C, Robinson S, Moser E. 2006. The selection of intended actions and the observation of others' actions: a time-resolved fMRI study. Neuroimage. 29:1294-302.

Curtis VA, Bullmore ET, Brammer MJ, Wright IC, Williams SC, Morris RG, Sharma TS, Murray RM, McGuire PK. 1998. Attenuated frontal activation during a verbal fluency task in patients with schizophrenia. American Journal of Psychiatry. 155:1056-63.

Daniel R, Wagner G, Koch K, Reichenbach JR, Sauer H, Schlösser RG. 2011. Assessing the neural basis of uncertainty in perceptual category learning through varying levels of distortion. Journal of Cognitive Neuroscience. 23:1781-93.

Das P, Lagopoulos J, Coulston CM, Henderson AF, Malhi GS. 2012. Mentalizing impairment in schizophrenia: a functional MRI study. Schizophrenia Research. 134:15864.

Dassonville P, Lewis SM, Zhu XH, Ugurbil K, Kim SG, Ashe J. 2001. The effect of stimulus-response compatibility on cortical motor activation. Neuroimage. 13:1-4.

David N, Bewernick BH, Cohen MX, Newen A, Lux S, Fink GR, Shah NJ, Vogeley K. 2006. Neural representations of self versus other: visual-spatial perspective taking and agency in a virtual ball-tossing game. Journal of cognitive neuroscience. 18:898-910.

Davis N, Cannistraci CJ, Rogers BP, Gatenby JC, Fuchs LS, Anderson AW, Gore JC. 2009. The neural correlates of calculation ability in children: an fMRI study. Magnetic resonance imaging. 27:1187-97.

De Bleser R, Dupont P, Postler J, Bormans G, Speelman D, Mortelmans L, Debrock M. 2003. The organisation of the bilingual lexicon: A PET study. Journal of Neurolinguistics. 16:439-56.

Decety J, Perani D, Jeannerod M, Bettinardi V, Tadary B, Woods R, Mazziotta JC, Fazio F. 1994. Mapping motor representations with positron emission tomography. Nature. 371:600.

Dehaene S, Naccache L, Cohen L, Le Bihan D, Mangin JF, Poline JB, Rivière D. 2001. Cerebral mechanisms of word masking and unconscious repetition priming. Nature Neuroscience. 4:752.

Delazer M, Domahs F, Bartha L, Brenneis C, Lochy A, Trieb T, Benke T. 2003. Learning complex arithmetic - an fMRI study. Cognitive Brain Research. 18:76-88.

Démonet JF, Price C, Wise R, Frackowiak RS. 1994. A PET study of cognitive strategies in normal subjects during language tasks: Influence of phonetic ambiguity and sequence processing on phoneme monitoring. Brain. 117:671-82.

Desai R, Conant LL, Waldron E, Binder JR. 2006. FMRI of past tense processing: the effects of phonological complexity and task difficulty. Journal of Cognitive Neuroscience. 18:278-97.

Devlin JT, Matthews PM, Rushworth MF. 2003. Semantic processing in the left inferior 
prefrontal cortex: a combined functional magnetic resonance imaging and transcranial magnetic stimulation study. Journal of cognitive neuroscience. 15:71-84.

Dichter GS, Belger A. Social stimuli interfere with cognitive control in autism. 2007. Neuroimage. 35:1219-30.

Diedrichsen J, Grafton S, Albert N, Hazeltine E, Ivry RB. 2006. Goal-selection and movement-related conflict during bimanual reaching movements. Cerebral Cortex. 16:1729-38.

Dolcos F, LaBar KS, Cabeza R. 2004. Dissociable effects of arousal and valence on prefrontal activity indexing emotional evaluation and subsequent memory: an eventrelated fMRI study. Neuroimage. 23:64-74.

Dupont S, Samson Y, Le Bihan D, Baulac M. 2003. Anatomy of verbal memory: a functional MRI study. Surgical and Radiologic Anatomy. 24:57-63.

Ernst M, Nelson EE, McClure EB, Monk CS, Munson S, Eshel N, Zarahn E, Leibenluft E, Zametkin A, Towbin K, Blair J. 2004. Choice selection and reward anticipation: an fMRI study. Neuropsychologia. 42:1585-97.

Ersner-Hershfield H, Wimmer GE, Knutson B. 2008. Saving for the future self: Neural measures of future self-continuity predict temporal discounting. Social cognitive and affective neuroscience. 4:85-92.

Fernández-Corcuera P, Salvador R, Monté GC, Sarró SS, Goikolea JM, Amann B, Moro N, Sans-Sansa B, Ortiz-Gil J, Vieta E, Maristany T. 2013. Bipolar depressed patients show both failure to activate and failure to de-activate during performance of a working memory task. Journal of Affective Disorders. 148:170-8.

Ferrandez AM, Hugueville L, Lehéricy S, Poline JB, Marsault C, Pouthas V. 2003. Basal ganglia and supplementary motor area subtend duration perception: an fMRI study. Neuroimage. 19:1532-44.

Fincham JM, Carter CS, Van Veen V, Stenger VA, Anderson JR. 2002. Neural mechanisms of planning: a computational analysis using event-related fMRI. Proceedings of the National Academy of Sciences. 99:3346-51.

Fitzgerald PB, Srithiran A, Benitez J, Daskalakis ZZ, Oxley TJ, Kulkarni J, Egan GF. 2008. An fMRI study of prefrontal brain activation during multiple tasks in patients with major depressive disorder. Human Brain Mapping. 29:490-501.

Fleming SM, Whiteley L, Hulme OJ, Sahani M, Dolan RJ. 2010. Effects of category-specific costs on neural systems for perceptual decision-making. Journal of Neurophysiology. 103:3238-47.

Forn C, Barros-Loscertales A, Escudero J, Benlloch V, Campos S, Antònia Parcet M, Avila C. 2007. Compensatory activations in patients with multiple sclerosis during preserved performance on the auditory N-back task. Human Brain Mapping. 28:424-30.

Frankenstein UN, Richter W, McIntyre MC, Remy F. 2001. Distraction modulates anterior cingulate gyrus activations during the cold pressor test. Neuroimage. 14:827-36.

Garavan H, Kelley D, Rosen A, Rao SM, Stein EA. Practice-related functional activation changes in a working memory task. 2000. Microscopy research and technique. 51:54-63.

Garavan H, Ross TJ, Li SJ, Stein EA. 2000. A parametric manipulation of central executive functioning. Cerebral cortex. 10:585-92.

Gemar MC, Kapur S, Segal ZV, Brown GM, Houle S. 1996. Effects of self-generated sad mood on regional cerebral activity: A PET study in normal subjects. Depression. 4:81-8.

Giesbrecht B, Woldorff MG, Song AW, Mangun GR. 2003. Neural mechanisms of top-down control during spatial and feature attention. Neuroimage. 19:496-512.

Grahn JA, Rowe JB. 2009. Feeling the beat: premotor and striatal interactions in musicians and nonmusicians during beat perception. Journal of Neuroscience. 29:7540-8.

Gray JR, Chabris CF, Braver TS. 2003. Neural mechanisms of general fluid intelligence. 
Nature Neuroscience. 6:316.

Griffiths TD, Rees G, Rees A, Green GG, Witton C, Rowe D, Büchel C, Turner R, Frackowiak RS. 1998. Right parietal cortex is involved in the perception of sound movement in humans. Nature Neuroscience. 1:74.

Grimm S, Schmidt CF, Bermpohl F, Heinzel A, Dahlem Y, Wyss M, Hell D, Boesiger P, Boeker H, Northoff G. 2006. Segregated neural representation of distinct emotion dimensions in the prefrontal cortex — an fMRI study. Neuroimage. 30:325-40.

Gruber O, Tost H, Henseler I, Schmael C, Scherk H, Ende G, Ruf M, Falkai P, Rietschel M. 2010. Pathological amygdala activation during working memory performance: evidence for a pathophysiological trait marker in bipolar affective disorder. Human Brain Mapping. 31:115-25.

Gruber O, von Cramon DY. 2003. The functional neuroanatomy of human working memory revisited: Evidence from 3-T fMRI studies using classical domain-specific interference tasks. Neuroimage. 19:797-809.

Gurd JM, Amunts K, Weiss PH, Zafiris O, Zilles K, Marshall JC, Fink GR. 2002. Posterior parietal cortex is implicated in continuous switching between verbal fluency tasks: an fMRI study with clinical implications. Brain. 125:1024-38.

Gutchess AH, Schacter DL. 2012. The neural correlates of gist-based true and false recognition. Neuroimage. 59:3418-26.

Hamzei F, Rijntjes M, Dettmers C, Glauche V, Weiller C, Büchel C. 2003. The human action recognition system and its relationship to Broca's area: an fMRI study. Neuroimage. 19:637-44.

Hartstra E, Oldenburg JF, Van Leijenhorst L, Rombouts SA, Crone EA. 2010. Brain regions involved in the learning and application of reward rules in a two-deck gambling task. Neuropsychologia. 48:1438-46.

Hauk O, Davis MH, Kherif F, Pulvermüller F. 2008. Imagery or meaning? Evidence for a semantic origin of category-specific brain activity in metabolic imaging. European Journal of Neuroscience. 27:1856-66.

Heckers S, Weiss AP, Deckersbach T, Goff DC, Morecraft RJ, Bush G. 2004. Anterior cingulate cortex activation during cognitive interference in schizophrenia. American Journal of Psychiatry. 161:707-15.

Heim ST, Opitz B, Friederici AD. 2002. Broca's area in the human brain is involved in the selection of grammatical gender for language production: evidence from event-related functional magnetic resonance imaging. Neuroscience letters. 328:101-4.

Herpertz SC, Huebner T, Marx I, Vloet TD, Fink GR, Stoecker T, Jon Shah N, Konrad K, Herpertz-Dahlmann B. 2008. Emotional processing in male adolescents with childhoodonset conduct disorder. Journal of Child Psychology and Psychiatry. 49:781-91.

Hofer A, Weiss EM, Golaszewski SM, Siedentopf CM, Brinkhoff C, Kremser C, Felber S, Fleischhacker WW. 2002. An FMRI study of episodic encoding and recognition of words in patients with schizophrenia in remission. American Journal of Psychiatry. 160:911-8.

Huang CM, Polk TA, Goh JO, Park DC. 2012. Both left and right posterior parietal activations contribute to compensatory processes in normal aging. Neuropsychologia. 50:55-66.

Iadarola MJ, Berman KF, Zeffiro TA, Byas-Smith MG, Gracely RH, Max MB, Bennett GJ. 1998. Neural activation during acute capsaicin-evoked pain and allodynia assessed with PET. Brain: a journal of neurology. 121:931-47.

Ino T, Doi T, Kimura T, Ito J, Fukuyama H. 2004. Neural substrates of the performance of an auditory verbal memory: between-subjects analysis by fMRI. Brain research bulletin. 64:115-26. 
Iseki K, Hanakawa T, Shinozaki J, Nankaku M, Fukuyama H. 2008. Neural mechanisms involved in mental imagery and observation of gait. Neuroimage. 41:1021-31.

Jabbi M, Swart M, Keysers C. 2007. Empathy for positive and negative emotions in the gustatory cortex. Neuroimage. 34:1744-53.

Jackson III O, Schacter DL. 2004. Encoding activity in anterior medial temporal lobe supports subsequent associative recognition. Neuroimage. 21:456-62.

Jacobsen T, Schubotz RI, Höfel L, Cramon DY. 2006. Brain correlates of aesthetic judgment of beauty. Neuroimage. 29:276-85.

Jobard G, Vigneau M, Mazoyer B, Tzourio-Mazoyer N. 2007. Impact of modality and linguistic complexity during reading and listening tasks. Neuroimage. 34:784-800.

Johnson SH, Rotte M, Grafton ST, Hinrichs H, Gazzaniga MS, Heinze HJ. 2002. Selective activation of a parietofrontal circuit during implicitly imagined prehension. Neuroimage. 17:1693-704.

Jonides J, Schumacher EH, Smith EE, Lauber EJ, Awh E, Minoshima S, Koeppe RA. 1997. Verbal working memory load affects regional brain activation as measured by PET. Journal of cognitive neuroscience. 9:462-75.

Kadosh RC, Henik A, Rubinsten O, Mohr H, Dori H, van de Ven V, Zorzi M, Hendler T, Goebel R, Linden DE. 2005. Are numbers special?: the comparison systems of the human brain investigated by fMRI. Neuropsychologia. 43:1238-48.

Kapur S, Tulving E, Cabeza R, McIntosh AR, Houle S, Craik FI. 1996. The neural correlates of intentional learning of verbal materials: a PET study in humans. Cognitive Brain Research. 4:243-9.

Kato J, Ide H, Kabashima I, Kadota H, Takano K, Kansaku K. 2009. Neural correlates of attitude change following positive and negative advertisements. Frontiers in behavioral neuroscience. 3:6.

Kawashima R, Satoh K, Itoh H, Ono S, Furumoto S, Gotoh R, Koyama M, Yoshioka S, Takahashi T, Takahashi K, Yanagisawa T. 1996. Functional anatomy of GO/NO-GO discrimination and response selection — a PET study in man. Brain research. 728:79-89.

Kertzman C, Schwarz U, Zeffiro TA, Hallett M. 1997. The role of posterior parietal cortex in visually guided reaching movements in humans. Experimental Brain Research. 114:17083.

Kim YH, Gitelman DR, Nobre AC, Parrish TB, LaBar KS, Mesulam MM. 1999. The largescale neural network for spatial attention displays multifunctional overlap but differential asymmetry. Neuroimage. 9:269-77.

Knutson B, Wimmer GE, Rick S, Hollon NG, Prelec D, Loewenstein G. 2008. Neural antecedents of the endowment effect. Neuron. 58:814-22.

Köhler S, Moscovitch M, Winocur G, Houle S, McIntosh AR. 1998. Networks of domainspecific and general regions involved in episodic memory for spatial location and object identity. Neuropsychologia. 36:129-42.

Kong J, Wang C, Kwong K, Vangel M, Chua E, Gollub R. 2005. The neural substrate of arithmetic operations and procedure complexity. Cognitive Brain Research. 22:397-405.

Konishi S, Hayashi T, Uchida I, Kikyo H, Takahashi E, Miyashita Y. 2002. Hemispheric asymmetry in human lateral prefrontal cortex during cognitive set shifting. Proceedings of the National Academy of Sciences. 99:7803-8.

Konishi S, Jimura K, Asari T, Miyashita Y. 2003. Transient activation of superior prefrontal cortex during inhibition of cognitive set. Journal of Neuroscience. 23:7776-82.

Koshino H, Kana RK, Keller TA, Cherkassky VL, Minshew NJ, Just MA. 2007. fMRI investigation of working memory for faces in autism: visual coding and underconnectivity with frontal areas. Cerebral Cortex. 18:289-300.

Kosslyn SM, DiGirolamo GJ, Thompson WL, Alpert NM. 1998. Mental rotation of objects 
versus hands: Neural mechanisms revealed by positron emission tomography. Psychophysiology. 35:151-61.

Kranz F, Ishai A. Face perception is modulated by sexual preference. 2006. Current biology. 16:63-8.

Kraut MA, Pitcock JA, Calhoun V, Li J, Freeman T, Hart Jr J. 2006. Neuroanatomic organization of sound memory in humans. Journal of cognitive neuroscience. 18:187788.

Kuo WJ, Yeh TC, Duann JR, Wu YT, Ho LT, Hung D, Tzeng OJ, Hsieh JC. 2001. A leftlateralized network for reading Chinese words: a 3 T fMRI study. Neuroreport. 12:39974001.

Kuo WJ, Yeh TC, Lee CY, Wu YT, Chou CC, Ho LT, Hung DL, Tzeng OJ, Hsieh JC. 2003. Frequency effects of Chinese character processing in the brain: an event-related fMRI study. Neuroimage. 18:720-30.

Kuo WJ, Yeh TC, Lee JR, Chen LF, Lee PL, Chen SS, Ho LT, Hung DL, Tzeng OJ, Hsieh JC. 2004. Orthographic and phonological processing of Chinese characters: an fMRI study. Neuroimage. 21:1721-31.

LaBar KS, Gitelman DR, Parrish TB, Mesulam MM. 1999. Neuroanatomic overlap of working memory and spatial attention networks: a functional MRI comparison within subjects. Neuroimage. 10:695-704.

Lai X, Zhang G, Huang Y, Tang C, Yang J, Wang S, Zhou SF. 2009. A cerebral functional imaging study by positron emission tomography in healthy volunteers receiving true or sham acupuncture needling. Neuroscience letters. 452:194-9.

Landau SM, Schumacher EH, Garavan H, Druzgal TJ, D'esposito M. 2004. A functional MRI study of the influence of practice on component processes of working memory. Neuroimage. 22:211-21.

Lanius RA, Williamson PC, Bluhm RL, Densmore M, Boksman K, Neufeld RW, Gati JS, Menon RS. 2005. Functional connectivity of dissociative responses in posttraumatic stress disorder: a functional magnetic resonance imaging investigation. Biological Psychiatry. 57:873-84.

Lanius RA, Williamson PC, Densmore M, Boksman K, Neufeld RW, Gati JS, Menon RS. 2004. The nature of traumatic memories: a 4-T FMRI functional connectivity analysis. American Journal of Psychiatry. 161:36-44.

Leibenluft E, Gobbini MI, Harrison T, Haxby JV. 2004. Mothers' neural activation in response to pictures of their children and other children. Biological Psychiatry. 56:22532.

Liljeström M, Tarkiainen A, Parviainen T, Kujala J, Numminen J, Hiltunen J, Laine M, Salmelin R. Perceiving and naming actions and objects. 2008. Neuroimage. 41:1132-41.

LoPresti ML, Schon K, Tricarico MD, Swisher JD, Celone KA, Stern CE. 2008. Working memory for social cues recruits orbitofrontal cortex and amygdala: a functional magnetic resonance imaging study of delayed matching to sample for emotional expressions. Journal of Neuroscience. 28:3718-28.

Lutz J, Herwig U, Opialla S, Hittmeyer A, Jäncke L, Rufer M, Grosse Holtforth M, Brühl AB. Mindfulness and emotion regulation - an fMRI study. 2013. Social Cognitive and Affective Neuroscience. 9:776-85.

Malik S, McGlone F, Bedrossian D, Dagher A. 2008. Ghrelin modulates brain activity in areas that control appetitive behavior. Cell Metabolism. 7:400-9.

Malisza KL, Allman AA, Shiloff D, Jakobson L, Longstaffe S, Chudley AE. 2005.

Evaluation of spatial working memory function in children and adults with fetal alcohol spectrum disorders: a functional magnetic resonance imaging study. Pediatric Research. 58:1150. 
Manoach DS, Gollub RL, Benson ES, Searl MM, Goff DC, Halpern E, Saper CB, Rauch SL. 2000. Schizophrenic subjects show aberrant fMRI activation of dorsolateral prefrontal cortex and basal ganglia during working memory performance. Biological psychiatry. 48:99-109.

Marangolo P, Piras F, Galati G, Burani C. 2006. Functional anatomy of derivational morphology. Cortex. 42:1093-106.

Maril A, Simons JS, Mitchell JP, Schwartz BL, Schacter DL. 2003. Feeling-of-knowing in episodic memory: an event-related fMRI study. Neuroimage. 18:827-36.

Marsh AA, Blair KS, Vythilingam M, Busis S, Blair RJ. 2007. Response options and expectations of reward in decision-making: the differential roles of dorsal and rostral anterior cingulate cortex. Neuroimage. 35:979-88.

Martin R, Barr A, MacIntosh B, Smith R, Stevens T, Taves D, Gati J, Menon R, Hachinski V. 2007. Cerebral cortical processing of swallowing in older adults. Experimental brain research. 176:12-22.

Martinkauppi S, Rämä P, Aronen HJ, Korvenoja A, Carlson S. 2000. Working memory of auditory localization. Cerebral Cortex. 10:889-98.

Marvel CL, Desmond JE. 2012. From storage to manipulation: how the neural correlates of verbal working memory reflect varying demands on inner speech. Brain and Language. 120:42-51.

Mazard A, Mazoyer B, Etard O, Tzourio-Mazoyer N, Kosslyn SM, Mellet E. 2002. Impact of fMRI acoustic noise on the functional anatomy of visual mental imagery. Journal of Cognitive Neuroscience. 14:172-86.

McNab F, Leroux G, Strand F, Thorell L, Bergman S, Klingberg T. 2008. Common and unique components of inhibition and working memory: an fMRI, within-subjects investigation. Neuropsychologia. 46:2668-82.

Mead LA, Mayer AR, Bobholz JA, Woodley SJ, Cunningham JM, Hammeke TA, Rao SM. 2002. Neural basis of the Stroop interference task: response competition or selective attention?. Journal of the International Neuropsychological Society. 8:735-42.

Mechelli A, Friston KJ, Price CJ. 2000. The effects of presentation rate during word and pseudoword reading: a comparison of PET and fMRI. Journal of Cognitive Neuroscience. 12(Supplement 2):145-56.

Mecklinger A, Weber K, Gunter TC, Engle RW. 2003. Dissociable brain mechanisms for inhibitory control: effects of interference content and working memory capacity. Cognitive Brain Research. 18:26-38.

Meisenzahl EM, Scheuerecker J, Zipse M, Ufer S, Wiesmann M, Frodl T, Koutsouleris N, Zetzsche T, Schmitt G, Riedel M, Spellmann I. 2006. Effects of treatment with the atypical neuroleptic quetiapine on working memory function: a functional MRI followup investigation. European Archives of Psychiatry and Clinical Neuroscience. 256:52231.

Meister IG, Iacoboni M. 2007. No language-specific activation during linguistic processing of observed actions. PLoS One. 2:e891.

Mickley Steinmetz KR, Kensinger EA. The effects of valence and arousal on the neural activity leading to subsequent memory. 2009. Psychophysiology. 46:1190-9.

Milham MP, Banich MT, Webb A, Barad V, Cohen NJ, Wszalek T, Kramer AF. 2001. The relative involvement of anterior cingulate and prefrontal cortex in attentional control depends on nature of conflict. Cognitive Brain Research. 12:467-73.

Milham MP, Banich MT. 2005. Anterior cingulate cortex: an fMRI analysis of conflict specificity and functional differentiation. Human brain mapping. 25:328-35.

Miura N, Watanabe J, Iwata K, Sassa Y, Riera J, Tsuchiya H, Sato S, Horie K, Takahashi M, Kitamura M, Kawashima R. 2005. Cortical activation during reading of ancient versus 
modern Japanese texts: fMRI study. Neuroimage. 26:426-31.

Mo L, Liu HL, Jin H, Yang YL. 2005. Brain activation during semantic judgment of Chinese sentences: A functional MRI study. Human brain mapping. 24:305-12.

Monchi O, Petrides M, Petre V, Worsley K, Dagher A. 2001. Wisconsin Card Sorting revisited: distinct neural circuits participating in different stages of the task identified by event-related functional magnetic resonance imaging. Journal of Neuroscience. 21:773341.

Monks PJ, Thompson JM, Bullmore ET, Suckling J, Brammer MJ, Williams SC, Simmons A, Giles N, Lloyd AJ, Louise Harrison C, Seal M. 2004. A functional MRI study of working memory task in euthymic bipolar disorder: evidence for task-specific dysfunction. Bipolar Disorders. 6:550-64.

Montojo CA, Courtney SM. 2008. Differential neural activation for updating rule versus stimulus information in working memory. Neuron. 59:173-82.

Nagahama Y, Okada T, Katsumi Y, Hayashi T, Yamauchi H, Oyanagi C, Konishi J, Fukuyama H, Shibasaki H. 2011. Dissociable mechanisms of attentional control within the human prefrontal cortex. Cerebral Cortex. 11:85-92.

Nakamura K, Kawashima R, Sato N, Nakamura A, Sugiura M, Kato T, Hatano K, Ito K, Fukuda H, Schormann T, Zilles K. 2000. Functional delineation of the human occipitotemporal areas related to face and scene processing: a PET study. Brain. 123:1903-12.

Nakao T, Nakagawa A, Yoshiura T, Nakatani E, Nabeyama M, Yoshizato C, Kudoh A, Tada K, Yoshioka K, Kawamoto M. 2005. A functional MRI comparison of patients with obsessive-compulsive disorder and normal controls during a Chinese character Stroop task. Psychiatry Research: Neuroimaging. 139:101-14.

Neuner I, Stöcker T, Kellermann T, Kircher T, Zilles K, Schneider F, Shah NJ. 2007. Wechsler Memory Scale Revised Edition: neural correlates of the visual paired associates subtest adapted for fMRI. Brain research. 1177:66-78.

Newman SD, Willoughby G, Pruce B. 2011. The effect of problem structure on problemsolving: an fMRI study of word versus number problems. Brain Research. 1410:77-88.

Noppeney U, Price CJ. Retrieval of abstract semantics. Neuroimage. 2004. 22:164-70.

Noriuchi M, Kikuchi Y, Senoo A. The functional neuroanatomy of maternal love: mother's response to infant's attachment behaviors. Biological psychiatry. 2008. 63:415-23.

Norris DG, Zysset S, Mildner T, Wiggins CJ. 2002. An investigation of the value of spinecho-based fMRI using a Stroop color-word matching task and EPI at 3 T. Neuroimage. 15:719-26.

Nunez JM, Casey BJ, Egner T, Hare T, Hirsch J. 2005. Intentional false responding shares neural substrates with response conflict and cognitive control. Neuroimage. 25:267-77.

Nunn JA, Gregory LJ, Brammer M, Williams SC, Parslow DM, Morgan MJ, Morris RG, Bullmore ET, Baron-Cohen S, Gray JA. 2002. Functional magnetic resonance imaging of synesthesia: activation of V4/V8 by spoken words. Nature Neuroscience. 5:371.

O'sullivan EP, Jenkins IH, Henderson L, Kennard C, Brooks DJ. 1995. The functional anatomy of remembered saccades: a PET study. Neuroreport. 6:2141-4.

Ochsner KN, Beer JS, Robertson ER, Cooper JC, Gabrieli JD, Kihsltrom JF, D'Esposito M. 2005. The neural correlates of direct and reflected self-knowledge. Neuroimage. 28:797814.

Ogawa H, Wakita M, Hasegawa K, Kobayakawa T, Sakai N, Hirai T, Yamashita Y, Saito S. 2005. Functional MRI detection of activation in the primary gustatory cortices in humans. Chemical Senses. 30:583-92.

Omori M, Yamada H, Murata T, Sadato N, Tanaka M, Ishii Y, Isaki K, Yonekura Y. 1999. Neuronal substrates participating in attentional set-shifting of rules for visually guided motor selection: a functional magnetic resonance imaging investigation. Neuroscience 
research. 33:317-23.

Papathanassiou D, Etard O, Mellet E, Zago L, Mazoyer B, Tzourio-Mazoyer N. 2000. A common language network for comprehension and production: a contribution to the definition of language epicenters with PET. Neuroimage. 11:347-57.

Parsons MW, Harrington DL, Rao SM. 2005. Distinct neural systems underlie learning visuomotor and spatial representations of motor skills. Human Brain Mapping. 24:22947.

Paulesu E, Perani D, Blasi V, Silani G, Borghese NA, De Giovanni U, Sensolo S, Fazio F. 2003. A functional-anatomical model for lipreading. Journal of Neurophysiology. 90:2005-13.

Pesenti M, Thioux M, Seron X, Volder AD. 2000. Neuroanatomical substrates of Arabic number processing, numerical comparison, and simple addition: A PET study. Journal of cognitive neuroscience. 12:461-79.

Peters J, Büchel C. 2010. Episodic future thinking reduces reward delay discounting through an enhancement of prefrontal-mediotemporal interactions. Neuron. 66:138-48.

Peters J, Büchel C. 2009. Overlapping and distinct neural systems code for subjective value during intertemporal and risky decision making. Journal of Neuroscience. 29:15727-34.

Petit L, Dubois S, Tzourio N, Dejardin S, Crivello F, Michel C, Etard O, Denise P, Roucoux and A, Mazoyer B. 1999. PET study of the human foveal fixation system. Human Brain Mapping. 8:28-43.

Phillips ML, Williams LM, Heining M, Herba CM, Russell T, Andrew C, Bullmore ET, Brammer MJ, Williams SC, Morgan M, Young AW. 2004. Differential neural responses to overt and covert presentations of facial expressions of fear and disgust. Neuroimage. 21:1484-96.

Pierce K, Haist F, Sedaghat F, Courchesne E. 2004. The brain response to personally familiar faces in autism: findings of fusiform activity and beyond. Brain. 127:2703-16.

Pihlajamäki M, Tanila H, Hänninen T, Könönen M, Laakso M, Partanen K, Soininen H, Aronen HJ. 2000. Verbal fluency activates the left medial temporal lobe: a functional magnetic resonance imaging study. Annals of Neurology: Official Journal of the American Neurological Association and the Child Neurology Society. 47:470-6.

Poeppel D, Guillemin A, Thompson J, Fritz J, Bavelier D, Braun AR. 2004. Auditory lexical decision, categorical perception, and FM direction discrimination differentially engage left and right auditory cortex. Neuropsychologia. 42:183-200.

Poldrack RA, Prabhakaran V, Seger CA, Gabrieli JD. 1999. Striatal activation during acquisition of a cognitive skill. Neuropsychology. 13:564.

Poldrack RA, Sabb FW, Foerde K, Tom SM, Asarnow RF, Bookheimer SY, Knowlton BJ. 2005. The neural correlates of motor skill automaticity. Journal of Neuroscience. 25:5356-64.

Pompei F, Jogia J, Tatarelli R, Girardi P, Rubia K, Kumari V, Frangou S. 2011. Familial and disease specific abnormalities in the neural correlates of the Stroop Task in Bipolar Disorder. Neuroimage. 56:1677-84.

Postle BR, Hamidi M. Nonvisual codes and nonvisual brain areas support visual working memory. Cerebral Cortex. 2006. 17:2151-62.

Ragland JD, Gur RC, Valdez JN, Loughead J, Elliott M, Kohler C, Kanes S, Siegel SJ, Moelter ST, Gur RE. 2005. Levels-of-processing effect on frontotemporal function in schizophrenia during word encoding and recognition. American Journal of Psychiatry. 162:1840-8.

Ranganath C, Yonelinas AP, Cohen MX, Dy CJ, Tom SM, D’Esposito M. 2005. Dissociable correlates of recollection and familiarity within the medial temporal lobes.

Neuropsychologia. 42:2-13. 
Ravnkilde B, Videbech P, Rosenberg R, Gjedde A, Gade A. 2002. Putative tests of frontal lobe function: a PET-study of brain activation during Stroop's Test and verbal fluency. Journal of Clinical and Experimental Neuropsychology. 24:534-47.

Rivera SM, Menon V, White CD, Glaser B, Reiss AL. 2002. Functional brain activation during arithmetic processing in females with fragile $\mathrm{X}$ Syndrome is related to FMR1 protein expression. Human brain mapping. 16:206-18.

Rossell SL, Bullmore ET, Williams SC, David AS. 2001. Brain activation during automatic and controlled processing of semantic relations: a priming experiment using lexicaldecision. Neuropsychologia. 39:1167-76.

Rossell SL, Bullmore ET, Williams SC, David AS. 2002. Sex differences in functional brain activation during a lexical visual field task. Brain and Language. 80:97-105.

Ruff CC, Woodward TS, Laurens KR, Liddle PF. 2001. The role of the anterior cingulate cortex in conflict processing: evidence from reverse stroop interference. Neuroimage. $14: 1150-8$.

Sadato N, Okada T, Honda M, Matsuki KI, Yoshida M, Kashikura KI, Takei W, Sato T, Kochiyama T, Yonekura Y. 2004. Cross-modal integration and plastic changes revealed by lip movement, random-dot motion and sign languages in the hearing and deaf. Cerebral Cortex. 15:1113-22.

Saur D, Lange R, Baumgaertner A, Schraknepper V, Willmes K, Rijntjes M, Weiller C. 2006. Dynamics of language reorganization after stroke. Brain. 129:1371-84.

Schmidt D, Krause BJ, Mottaghy FM, Halsband U, Herzog H, Tellmann L, Müller-Gärtner HW. 2002. Brain systems engaged in encoding and retrieval of word-pair associates independent of their imagery content or presentation modalities. Neuropsychologia. 40:457-70.

Seo J, Kim SH, Kim YT, Song HJ, Lee JJ, Kim SH, Han SW, Nam EJ, Kim SK, Lee HJ, Lee SJ. 2012. Working memory impairment in fibromyalgia patients associated with altered frontoparietal memory network. PloS one. 7:e37808.

Sergerie K, Armony JL, Menear M, Sutton H, Lepage M. 2009. Influence of emotional expression on memory recognition bias in schizophrenia as revealed by fMRI. Schizophrenia Bulletin. 36:800-10.

Sergerie K, Lepage M, Armony JL. 2006. A process-specific functional dissociation of the amygdala in emotional memory. Journal of Cognitive Neuroscience. 18:1359-67.

Shergill SS, Bullmore ET, Brammer MJ, Williams SC, Murray RM, McGuire PK. 2001. A functional study of auditory verbal imagery. Psychological Medicine. 31:241-53.

Simmons WK, Hamann SB, Harenski CL, Hu XP, Barsalou LW. 2008. fMRI evidence for word association and situated simulation in conceptual processing. Journal of Physiology-Paris. 102(1-3):106-19.

Simões-Franklin C, Hester R, Shpaner M, Foxe JJ, Garavan H. 2010. Executive function and error detection: the effect of motivation on cingulate and ventral striatum activity. Human Brain Mapping. 31:458-69.

Small GW, Moody TD, Siddarth P, Bookheimer SY. 2009. Your brain on Google: patterns of cerebral activation during internet searching. The American Journal of Geriatric Psychiatry. 17:116-26.

Smith YR, Love T, Persad CC, Tkaczyk A, Nichols TE, Zubieta JK. 2006. Impact of combined estradiol and norethindrone therapy on visuospatial working memory assessed by functional magnetic resonance imaging. The Journal of Clinical Endocrinology \& Metabolism. 91:4476-81.

Sperling RA, Bates JF, Cocchiarella AJ, Schacter DL, Rosen BR, Albert MS. 2001. Encoding novel face-name associations: A functional MRI study. Human brain mapping. 14:12939. 
Sylvester CY, Wager TD, Lacey SC, Hernandez L, Nichols TE, Smith EE, Jonides J. 2003. Switching attention and resolving interference: fMRI measures of executive functions. Neuropsychologia. 41:357-70.

Tatsumi IF, Fushimi T, Sadato N, Kawashima R, Yokoyama E, Kanno I, Senda M. 1999. Verb generation in Japanese - a multicenter PET activation study. Neuroimage. 9:15464.

Thompson-Schill SL, Aguirre GK, Desposito M, Farah MJ. 1999. A neural basis for category and modality specificity of semantic knowledge. Neuropsychologia. 37:671-6.

Tremblay P, Gracco VL. 2006. Contribution of the frontal lobe to externally and internally specified verbal responses: fMRI evidence. Neuroimage. 33:947-57.

Tyler LK, Stamatakis EA, Post B, Randall B, Marslen-Wilson W. 2005. Temporal and frontal systems in speech comprehension: An fMRI study of past tense processing. Neuropsychologia. 43:1963-74.

Uchiyama H, Seki A, Kageyama H, Saito DN, Koeda T, Ohno K, Sadato N. 2006. Neural substrates of sarcasm: a functional magnetic-resonance imaging study. Brain Research. 1124:100-10.

Uher R, Treasure J, Heining M, Brammer MJ, Campbell IC. 2006. Cerebral processing of food-related stimuli: effects of fasting and gender. Behavioural brain research. 169:1119.

Van Boven RW, Ingeholm JE, Beauchamp MS, Bikle PC, Ungerleider LG. 2005. Tactile form and location processing in the human brain. Proceedings of the National Academy of Sciences of the United States of America. 102:12601-5.

van Leijenhorst L, Crone EA, Bunge SA. 2006. Neural correlates of developmental differences in risk estimation and feedback processing. Neuropsychologia. 44:2158-70.

Vitali P, Abutalebi J, Tettamanti M, Rowe J, Scifo P, Fazio F, Cappa SF, Perani D. 2005. Generating animal and tool names: An fMRI study of effective connectivity. Brain and Language. 93:32-45.

Voets NL, Adcock JE, Flitney DE, Behrens TE, Hart Y, Stacey R, Carpenter K, Matthews PM. 2005. Distinct right frontal lobe activation in language processing following left hemisphere injury. Brain. 129:754-66.

Vuilleumier P, Armony JL, Driver J, Dolan RJ. 2001. Effects of attention and emotion on face processing in the human brain: an event-related fMRI study. Neuron. 30:829-41.

Wagner AD, Paré-Blagoev EJ, Clark J, Poldrack RA. 2001. Recovering meaning: left prefrontal cortex guides controlled semantic retrieval. Neuron. 31:329-38.

Wang AT, Dapretto M, Hariri AR, Sigman M, Bookheimer SY. 2004. Neural correlates of facial affect processing in children and adolescents with autism spectrum disorder. Journal of the American Academy of Child \& Adolescent Psychiatry. 43:481-90.

Weiss EM, Golaszewski S, Mottaghy FM, Hofer A, Hausmann A, Kemmler G, Kremser C, Brinkhoff C, Felber SR, Fleischhacker WW. 2003. Brain activation patterns during a selective attention test - a functional MRI study in healthy volunteers and patients with schizophrenia. Psychiatry Research: Neuroimaging. 123:1-5.

Weiss EM, Hofer A, Golaszewski S, Siedentopf C, Brinkhoff C, Kremser C, Felber S, Fleischhacker WW. 2004. Brain activation patterns during a verbal fluency test-a functional MRI study in healthy volunteers and patients with schizophrenia. Schizophrenia Research. 70:287-91.

Wheaton KJ, Thompson JC, Syngeniotis A, Abbott DF, Puce A. 2004. Viewing the motion of human body parts activates different regions of premotor, temporal, and parietal cortex. Neuroimage. 22:277-88.

Wicker B, Keysers C, Plailly J, Royet JP, Gallese V, Rizzolatti G. 2003. Both of us disgusted in My insula: the common neural basis of seeing and feeling disgust. Neuron. 40:655-64. 
Wong PC, Uppunda AK, Parrish TB, Dhar S. 2008. Cortical mechanisms of speech perception in noise. Journal of Speech, Language, and Hearing Research. 51:1026-41.

Wong SW, Massé N, Kimmerly DS, Menon RS, Shoemaker JK. 2007. Ventral medial prefrontal cortex and cardiovagal control in conscious humans. Neuroimage. 35:698-708.

Wraga M, Thompson WL, Alpert NM, Kosslyn SM. 2003. Implicit transfer of motor strategies in mental rotation. Brain and Cognition. 52:135-43.

Xiang H, Lin C, Ma X, Zhang Z, Bower JM, Weng X, Gao JH. 2003. Involvement of the cerebellum in semantic discrimination: an fMRI study. Human Brain Mapping. 18:20814.

Xiong J, Rao S, Jerabek P, Zamarripa F, Woldorff M, Lancaster J, Fox PT. 2000. Intersubject variability in cortical activations during a complex language task. Neuroimage. 12:32639.

Xu B, Grafman J, Gaillard WD, Ishii K, Vega-Bermudez F, Pietrini P, Reeves-Tyer P, DiCamillo P, Theodore W. 2001. Conjoint and extended neural networks for the computation of speech codes: the neural basis of selective impairment in reading words and pseudowords. Cerebral Cortex. 11:267-77.

Xue G, Ghahremani DG, Poldrack RA. 2008. Neural substrates for reversing stimulusoutcome and stimulus-response associations. Journal of Neuroscience. 28:11196-204.

Yassa MA, Verduzco G, Cristinzio C, Bassett SS. 2008. Altered fMRI activation during mental rotation in those at genetic risk for Alzheimer disease. Neurology. 70:1898-904.

Yoo SS, Choi BG, Juh RH, Park JM, Pae CU, Kim JJ, Lee SJ, Lee C, Paik IH, Lee CU, Adkinson NF. 2005. Working memory processing of facial images in schizophrenia: fMRI investigation. International Journal of Neuroscience. 115:351-66.

Yoo SS, Hu PT, Gujar N, Jolesz FA, Walker MP. 2007. A deficit in the ability to form new human memories without sleep. Nature Neuroscience. 10:385.

Zago L, Pesenti M, Mellet E, Crivello F, Mazoyer B, Tzourio-Mazoyer N. 2001. Neural correlates of simple and complex mental calculation. Neuroimage. 13:314-27.

Zandbelt BB, van Buuren M, Kahn RS, Vink M. 2011. Reduced proactive inhibition in schizophrenia is related to corticostriatal dysfunction and poor working memory. Biological Psychiatry. 70:1151-8.

Zatorre RJ, Perry DW, Beckett CA, Westbury CF, Evans AC. 1998. Functional anatomy of musical processing in listeners with absolute pitch and relative pitch. Proceedings of the National Academy of Sciences. 95:3172-7.

Zhang M, Mariola E, Stilla R, Stoesz M, Mao H, Hu X, Sathian K. 2005. Tactile discrimination of grating orientation: fMRI activation patterns. Human Brain Mapping. 25:370-7.

Ziv M, Tomer R, Defrin R, Hendler T. 2010. Individual sensitivity to pain expectancy is related to differential activation of the hippocampus and amygdala. Human Brain Mapping. 31:326-38.

Zysset S, Müller K, Lohmann G, von Cramon DY. 2001. Color-word matching Stroop task: separating interference and response conflict. Neuroimage. 13:29-36. 